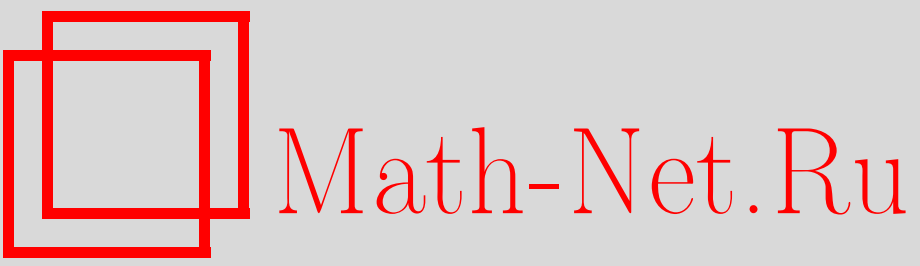

А. А. Борисенко, Изометрические погружения пространственных форм в римановы и псевдоримановы пространства постоянной кривизны, УМH, 2001, том 56, выпуск 3, 3-78

DOI: https://doi.org/10.4213/rm393

Использование Общероссийского математического портала Math-Net.Ru подразумевает, что вы прочитали и согласны с пользовательским соглашением

http://www.mathnet.ru/rus/agreement

Параметры загрузки:

IP: 54.81 .137 .203

26 апреля 2023 г., 10:32:13 


\section{ИЗОМЕТРИЧЕСКИЕ ПОГРУЖЕНИЯ ПРОСТРАНСТВЕННЫХ ФОРМ В РИМАНОВЫ И ПСЕВДОРИМАНОВЫ ПРОСТРАНСТВА ПОСТОЯННОЙ КРИВИЗНЫ}

\section{А. А. БОРИСЕНКО}

В обзоре изложены резултаты по локальному и глобальному изометрическому погружению двумерных и многомерных римановых и псевдоримановых пространственных форм в пространства постоянной кривизны.

Библиография: 178 названий.

\section{СОДЕРЖАНИЕ}

Введение

Глава 1. Изометрические погружения многомерных римановых многообразий

1.1. Локальные и глобальные результаты … . . . . . . . . . . . . . . . . . .

1.2. Гладкие поверхности ограниченной внешней кривизны $\ldots . . \ldots \ldots \ldots . . . .6$

1.3. Аффиннно-устойчивые погружения римановых метрик ............... 10

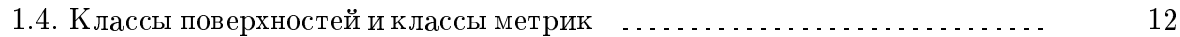

Глава 2. Изометрические регулярные погружения двумерных метрик

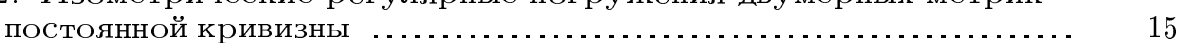

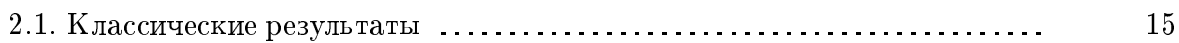

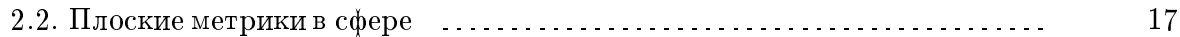

2.3. Изометрические погружения двумерных плоских метрик $\quad . . . . . . . . . .2$

2.4. Изометрические погружения областей плоскости Лобачевского . ....... 29

2.5. Изометрические погружения с постоянной внешней геометрией $\ldots . . . .32$

2.6. Погружение проективной плоскости .............................. 35

Глава 3. Изометрические погружения многомерных пространственных

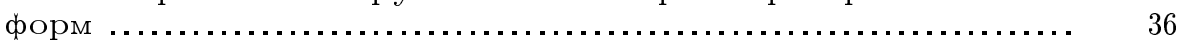

3.1. Изометрические погружения пространственных форм подмногообразия-

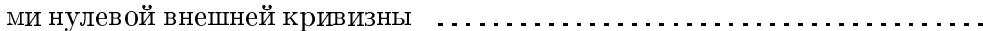

3.2. Изометрические погружения пространственных форм в качестве подмно-

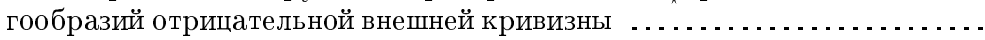

3.3. Изометрические погружения в виде подмногообразий положительной внешней кривизны

Глава 4. Изометрические погружения псевдоримановых пространств постоянной кривизны

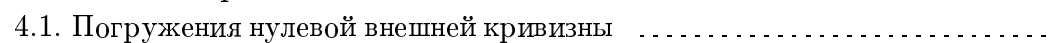

4.2. Изометрические погружения псевдоримановых пространств ненулевой постоянной кривизны 


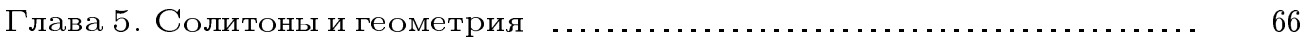

5.1. Солитоны и псевдосферические поверхности .................... 66

5.2. Изометрические погружения с дополнительньми ограничениями ...... 68

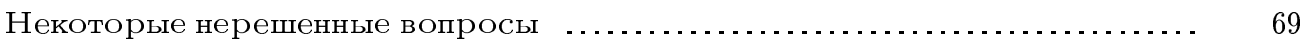

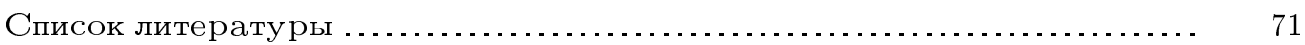

\section{Введение}

Изометрическому погружению многомерных римановых многообразий в евклидовы пространства посвящена обширная литература, в частности, обзоры М. Л. Громова, В.А. Рохлина [80], Ю.А. Аминова [10], Н. Джакобовича [94], Э.Г. Позняка, Д. Д. Соколова [138].

При большой коразмерности погружения имеется большая свобода и нет тесных связей между внутренней геометрией и внешней геометрией многообразия. Однако при изометрическом погружении пространств постоянной секционной кривизны с достаточно малой коразмерностью вложения возникают жесткие связи между внешней геометрией многообразия и ее кривизной. Некоторые вопросы в этой тематике еще не решены. В частности, еше не решен вопрос о многомерном обобшении теоремы Гильберта.

Этот обзор посвящен изложению локальных и глобальных результатов об изометрическом погружении римановых и псевдоримановых многообразий постоянной секционной кривизны.

В 1987 году Д.В. Алексеевский на конференщии в г. Новосибирске, посвященной 75-летию академика А. Д. Александрова, обратился ко мне с рядом вопросов по этой тематике. И у него возникла идея написания обзора в этом направлении. Наконец я реализовал этот замысел и сердечно благодарю Д. В. Алексеевского за эту идею. Благодарю также рецензента за замечания.

\section{Глава 1. Изометрические погружения многомерных римановых многообразий}

1.1. Локальные и глобальные результаты. В дифференциальной топологии отображение одного гладкого многообразия в другое назьвается вложением, если оно представляет собой диффеоморфизм на подмногообразие, и погружением, если оно является вложением локально, т.е. обладает (при должной гладкости) невырождающимся дифференциалом. В римановой геометрии они, кроме того, изометричные отображения.

Всякое гладкое многообразие размерности $n$ дифференциально вкладывается в $E^{2 n}$, а если $n-$ не степень двойки, то и в $E^{2 n-1}$. При любом $n$ в $E^{2 n-1}$ дифференциально вкладывается всякое гладкое многообразие размерности $n$ без замкнутых компонент, и при любом $n>1$ в $E^{2 n-1}$ дифференциально погружается всякое гладкое многообразие размерности $n$. Если $n$ - степень двойки, то существуют гладкие замкнутые многообразия размерности $n$, не вкладьваемые в $E^{2 n-1}$ ни дифференциально, ни топологически и не погружаемые в $E^{2 n-2}$, таково, например, вещественное проективное пространство $\mathbb{R} P^{n}$.

Для изометрических погружений была доказана 
Теорема 1.1.1 (Картан [45], Жане [96]). Во всяком аналитическом римановом многообразии размерности $n$ с отмеченной точкой существует окрестность отмеченной точки, допускающая изометрическое аналитическое вложение в $E^{s_{n}}, s_{n}=\frac{1}{2} n(n+1) ; s_{n}$ нельзя заменить на $s_{n}-1$.

Обозначим для $C^{2}$-отображения $f$ гладкого $C^{2}$-многообразия $V$ размерности $n$ в $E^{q}$ через $T_{v}^{2}(f)$ соприкасающееся пространство, натянутое на первые и вторые производные радиус-вектора $x=x(u)$ :

$$
\frac{\partial x}{\partial u_{i}}(i=1, \ldots, n), \quad \frac{\partial^{2} x}{\partial u_{i} \partial u_{j}}(i, j=1, \ldots, n),
$$

где $u=\left(u_{1}, \ldots, u_{n}\right)$ - координаты на $V$. Если $\operatorname{dim} T_{v}^{2}(f)=n+s_{n}$, то отображение называется свободным.

Теорема 1.1.2 (Громов, Рохлин [80]). В римановом многообразии размерности п и класса $C^{\infty}$ с отмеченной точкой существует окрестность отмеченной точки, допускающая свободное изометрическое вложение в $E^{s_{n}+n}$.

Теорема 1.1.3 (Джакобович [94]). Каждое $C^{l}$-регулярное риманово многообразие локально допускает $C^{l}$-регулярное изометрическое вложение в евклидово пространство $E^{s_{n}+n}$, где $l$ - любое вещественное число, $2<l \leqslant \infty$.

При ослабленных условиях регулярности некоторая окрестность каждой точки произвольного $C^{1}$-риманова $n$-мерного многообразия может быть $C^{1}$-изометрично вложена в евклидово пространство $E^{n+1}[107]$, [122].

Для глобальных изометрических погружений имеют место следуюшие теоремы.

Теорема 1.1.4 (Нэш, см. [117]). Всякое замкнутое риманово многообразие размерности $n$ и класса $C^{r}(3 \leqslant r \leqslant \infty)$ допускает изометрическое $C^{r}$-вложсение в $E^{q}, q=3 s_{n}+4 n=\frac{1}{2}\left(3 n^{2}+11 n\right)$.

Tеорема 1.1.5 (Громов, Рохлин, см. [117]). Компактное класса $C^{\infty}$ риманово многообразие допускает $C^{\infty}$-изометрическое свободное вложение в $E^{q}, q=s_{n}+$ $3 n+5$.

Для двумерных римановых многообразий имеют место более сильные результаты.

Всякое компактное двумерное риманово многообразие класса $C^{a}\left(C^{\infty}\right)$ допускает $C^{a}-\left(C^{\infty}-\right)$ вложение в $E^{10}$, если оно не имеет замкнутых компонент, риманово многообразие диффеоморфно сфере, изометрически вкладьвается в $E^{7}$ [80].

Непрерьвное отображение назьвается собственным, если прообразы компактных множеств компактны. Если отображение является топологическим вложением, то это условие эквивалентно замкнутости образа.

Если риманово многообразие допускает дифференциальное вложение в $E^{q}$, то оно допускает изометрическое $C^{1}$-вложение в $E^{q+1}[107]$, [122].

Сопоставляя этот факт с дифференциальной вложимостью гладких некомпактных связных $n$-мерных многообразий в $E^{2 n}$, мы получим, что всякое риманово многообразие размерности $n$ допускает изометрическое $C^{1}$-вложение в $E^{2 n+1}$.

$C^{1}$-ориентируемое двумерное риманово многообразие с конечнопорожденной фундаментальной группой допускает $C^{1}$-вложение в $E^{3}$. 
Ориентируемое (неориентируемое) полное двумерное риманово многообразие с конечной фундаментальной группой допускает изометрическое $C^{1}$-вложение $E^{3}\left(E^{4}\right)$ с замкнутым образом [80].

1.2. Гладкие поверхности ограниченной внешней кривизны. Качественное обилие погружений обеспечивается понижением гладкости или увеличением коразмерности погружения. Для многих таких погружений утрачивается как качественная связь строения поверхности со знаком ее кривизны, так и количественные связи ее внутренней и внешней геометрии.

В то же время можно привести примеры, когда изометрические погружения подчиняются некоторьм дополнительным требованиям "регулярности” и это обеспечивает достаточно тесную связь между внешней и внутренней геометрией погружения.

Примеры таких ограничений весьма разнообразны. Среди них встречаются как чисто дифференциальные, так и геометрические. Первой работой, в которой "регулярность” погружения понималась чисто геометрически (вьпуклость), является, по-видимому, работа А. Д. Александрова, посвященная проблеме Г. Вейля.

Гладкость погружения является следствием гладкости метрики и вьпуклости погружения. Это классический результат А. В. Погорелова.

Теорема 1.2.1 (Погорелов [131], [133]). Если выпуклая поверхность имеет регулярную метрику класса $C^{k}(k \geqslant 2)$ с гауссовой кривизной больше нуля, то сама поверхность принадлежит классу $C^{k-1, \beta}$ слюбым $\beta<1$.

Мы видим, что здесь гарантируемая гладкость погружения ниже гладкости погружаемой метрики. Однако оказьвается, что потерь гладкости при погружении нет.

Теорема 1.2.2 (Сабитов[142]). Выпуклая поверхность с $C^{k, \alpha}$-регулярной метрикой $(k \geqslant 2,0<\alpha<1)$ и положительной кривизной является $C^{k, \alpha}-$-ладкой.

Естественно возникает более обший вопрос: как связаны между собой регулярность метрики, индуцируемой погружением, и класс регулярности погружения. На первьй взгляд, при погружении регулярность метрики должна понижаться. Но в действительности это не так. Это показали И.Х. Сабитов и С.З. Шефель в работе [147]. Прежде всего там были введены гармонические координаты и с их помошюю была доказана

Теорема 1.2.3 (Сабитов, Шефель [147]). Всякая $C^{k, \alpha}$-гладкая $(k \geqslant 2,0<\alpha<1)$ l-мерная поверхность $F^{l}$ в римановом пространстве $M^{n}$, регулярности не ниже регулярности поверхности, является $C^{k, \alpha}-$ гладким изометрическим погружением некоторого $C^{k, \alpha}-$ гладкого риманова многообразия $M^{l}$.

Если гауссова кривизна поверхности неотрицательна, то доказаны более слабые результаты. Для $C^{n}$-гладких метрик с гауссовой кривизной $K \geqslant 0$ при $n \geqslant 10$ доказана локальная погружаемость в виде $C^{n-6}$-гладкой вьпуклой поверхности [110]. Если на сфере задана $C^{4}$-гладкая метрика с гауссовой кривизной $K \geqslant 0$, то сушествует $C^{1,1}$-изометричное вложение [89].

После работ Дж. Нэша и Н. Кейпера стало ясно, что требование $C^{1}$-гладкости изометричного погружения слишком слабое. При этом происходит нарушение связей 
между внутренней и внешней геометрией при малой коразмерности вложения. Любую гладкую поверхность $F$ в $E^{3}$ можно в классе изометричных поверхностей непрерьвно изогнуть в поверхность, лежашую в сколь угодно малом шаре. В частности, $C^{1}$-гладкая поверхность с аналитической метрикой положительной кривизны может не быть вьпуклой.

Совершенно ясно, что сохранить для гладких поверхностей даже со сколь угодно хорошей внутренней метрикой основную в теории поверхностей интегральную теорему о связи между внутренней и внешней кривизной в ее обычной формулировке невозможно.

Все это делает необходимым наложить на поверхность другие ограничения, касающиеся ее внешней геометрии. Наиболее естественным и геометричным является требование ограниченности площади сферического изображения, которое позволяет просто и непосредственно определить важнейшее в теории поверхностей понятие кривизны [132], [133].

Точное определение рассматриваемого класса поверхностей состоит в следующем. Пусть $\Phi$ - гладкая поверхность и $F_{1}, F_{2}, \ldots, F_{r}$ - любые попарно не пересекающиеся замкнутые множества на ней. Сферическое изображение $\bar{F}_{s}$ каждого множества $F_{s}$ является также замкнутым множеством, а следовательно, имеет определенную площадь $\mu\left(\bar{F}_{s}\right)$ (лебегову меру). Поверхность $\Phi$ назьвается поверхностью ограниченной внешней кривизны, если

$$
\mu\left(\bar{F}_{1}\right)+\mu\left(\bar{F}_{2}\right)+\cdots+\mu\left(\bar{F}_{r}\right)<c(\Phi)
$$

где $c(\Phi)$ - постоянная, не зависящая ни от множеств $F_{s}$, ни от их числа. Таким образом, это поверхность, у которой площадь сферического изображения с учетом кратности конечна.

Для поверхностей ограниченной внешней кривизны вводится понятие абсолютной внешней кривизны на произвольном множестве. Именно, если $G$ - открытое множество, $F_{1}, F_{2}, \ldots, F_{r}$ - замкнутые множества, содержашиеся в $G$, и $\mu\left(\bar{F}_{1}\right), \mu\left(\bar{F}_{2}\right), \ldots, \mu\left(\bar{F}_{r}\right)$ - плошади их сферических изображений, то под абсолютной кривизной поверхности на множестве $G$ понимаем

$$
\sigma^{0}(G)=\sup \sum_{s} \mu\left(\bar{F}_{s}\right)
$$

где sup берется по всем конечным системам замкнутых, попарно не пересекаюшихся множеств в $G$. Абсолютная кривизна на любом множестве $H$ определяется равенством

$$
\begin{gathered}
\sigma^{0}(H)=\inf \sigma^{0}(G), \\
H \subset G,
\end{gathered}
$$

где inf берется по всем открытым множествам $G$, содержашим $H$. Очевидно, если поверхность регулярна (дважды дифференцируема), то

$$
\sigma^{0}(H)=\int_{H}|K| d S
$$


где $K$ - гауссова кривизна поверхности, а $d S$ - элемент площади.

Определяемая указанным образом абсолютная кривизна поверхности обладает характерным для кривизны свойством полной аддитивности на кольце борелевских множеств.

Абсолютная кривизна поверхности допускает удобное во многих случаях интегральное представление для любого борелевского множества $H$ :

$$
\sigma^{0}(H)=\int_{\omega} n_{H}(X) d X
$$

где $n_{H}(X)$ - число точек в $H$, имеюших своим образом при сферическом отображении точку $X$ единичной сферы $\omega, d X$ - элемент площади сферы, а интегрирование распространяется на всю сферу.

Точки поверхности ограниченной внешней кривизны подразделяются на регулярные и нерегулярные. Точка $X$ называется регулярной, если в достаточно малой ее окрестности нет точек с касательньми плоскостями, параллельными касательной плоскости в точке $X$. Остальные точки поверхности назьваются нерегулярньми. Абсолютная кривизна поверхности на множестве нерегулярных точек равна нулю.

Регулярные точки поверхности в свою очередь подразделяются на эллиптические, гиперболические, параболические и точки уплощения в зависимости от характера пересечения касательной плоскости с поверхностью. Именно, если точка поверхности $X$ имеет окрестность $U(X)$ такую, что касательная плоскость в $X$ имеет с $U(X)$ единственную общую точку - точку $X$, то эта точка называется эллиптической. Если касательная плоскость в точке $X$ пересекает поверхность вблизи точки касания по двум простым кривым, исходяшим из $X$, то точка $X$ назьвается параболической точкой, если пересечение состоит из четырех кривых, то $X$ назьвается гиперболической точкой. В остальных случаях регулярных точек пересечение касательной плоскости с поверхностью вблизи точки касания состоит из четного числа, большего четырех, простых кривых, исходяших из точки касания. Все такие точки назьваются точками уплощения.

Приведенная классификация регулярных точек позволяет, естественно, определить понятие положительной, отрицательной и полной внешней кривизны поверхности на произвольном множестве. Именно, положительной (отрицательной) внешней кривизной поверхности на множестве $H$ называется абсолютная кривизна поверхности на подмножестве эллиптических (гиперболических) точек $H$. Полная внешняя кривизна на множестве $H$ определяется как разность между положительной и отрицательной кривизнами поверхности на этом множестве. Очевидно, если поверхность регулярна, то положительная, отрищательная и полная кривизны ее на множестве $H$, соответственно, равны:

$$
\sigma^{+}(H)=\int_{H(K>0)} K d S, \quad \sigma^{-}(H)=-\int_{H(K<0)} K d S, \quad \sigma(H)=\int_{H} K d S,
$$

где $K$ - гауссова кривизна поверхности, $d S$ - элемент плошади, а интегрирование в первых двух формулах распространяется на подмножества $H$, где $K>0$ и $K<0$ соответственно. 
Положительная, отрицательная и полная кривизны поверхности ограниченной внешней кривизны вполне аддитивны на кольце борелевских множеств поверхности.

Полная кривизна поверхности представляет собой алгебраическую площадь сферического изображения и для гомеоморфной кругу области $G$, ограниченной простой кривой $\gamma$ с равной нулю абсолютной кривизной, допускает представление:

$$
\sigma(G)=\int_{\omega-\bar{\gamma}} q_{\bar{\gamma}}(X) d X,
$$

где $q \bar{\gamma}(X)$ - степень точки $X$ единичной сферы относительно кривой $\bar{\gamma}-$ сферического изображения кривой $\gamma$, т.е. число обходов этой точки при движении вдоль кривой $\bar{\gamma}$; $d X$ - элемент площади сфреры.

Внешняя форма поверхности неотрищательной кривизны $\left(\sigma^{-}=0\right)$ и нулевой кривизны $\left(\sigma^{-}=\sigma^{+}=0\right)$ выясняется следуюшими теоремами.

ТЕОРема 1.2.4 (Погорелов [132]). Поверхность нулевой внешней кривизнь развертывающаяся, т.е. локально изометрична плоскости, и имеет обьчное для регулярных развертывающихся поверхностей строение с прямолинейными образующими и стационарной касательной плоскостью вдоль кажсой образующей. Полная поверхность нулевой кривизны иилиндрическая.

Теорема 1.2.5 (Погорелов [132]). Полная поверхность с неотричательной внешней кривизной и положительной кривизной, отличной от нуля, есть либо замкнутая выпуклая поверхность, либо бесконечная выпуклая поверхность.

Внутренне геометрическое исследование поверхности ограниченной внешней кривизны, а также установление связи между внутренней метрикой поверхности и ее внешней формой оказьвается возможным благодаря теореме о приближении такой поверхности регулярными. Именно: пусть $\Phi$ - поверхность ограниченной внешней кривизны и $X$ - произвольная точка на ней, тогда точка $X$ имеет окрестность $U(X)$ такую, что существует последовательность регулярных, дажсе аналитических, поверхностей $\Phi_{n}$ с равномерно ограниченными абсолютными кривизнами, сходящаяся $\kappa \Phi$ в $U(X)$ вместе со сферическими изображсениями.

Эта теорема позволяет заключить, что поверхности ограниченной внешней кривизны суть многообразия ограниченной внутренней кривизны в смысле А. Д. Александрова [3].

Таким образом, для поверхностей ограниченной внешней кривизны определены понятия внутренней положительной $\left(\omega^{+}\right)$, отрицательной $\left(\omega^{-}\right)$и полной $(\omega)$ кривизн. Для регулярных поверхностей связь между этими кривизнами и соответствующими внешними кривизнами устанавливается известной теоремой Гаусса. В случае поверхностей ограниченной внешней кривизны имеют место аналогичные теоремы.

Для любого борелевского множества $H$ на поверхности ограниченной внешней кривизны

$$
\omega^{+}(H)=\sigma^{+}(H), \quad \omega^{-}(H) \geqslant \sigma^{-}(H), \quad \omega(H) \leqslant \sigma(H) .
$$

Существует открытое множество $G$, содержащее все регулярные точки поверхности, такое, что для любого борелевского множества $H$ из $G$

$$
\omega^{+}(H)=\sigma^{+}(H), \quad \omega^{-}(H)=\sigma^{-}(H), \quad \omega(H)=\sigma(H) .
$$


Равенство внутренних и внешних кривизн имеет место для любого борелевского множества на замкнутой поверхности ограниченной внешней кривизны.

Рассмотрим поверхности с неотрицательной внутренней и ограниченной внешней кривизной. Начнем с поверхностей нулевой внутренней кривизны, т.е. поверхностей, у которых положительная и отрицательная внутренние кривизны равны нулю. На такой поверхности не может быть ни эллиптических, ни гиперболических точек. Действительно, если на поверхности есть гиперболическая точка, то отрицательная внешняя кривизна поверхности отлична от нуля. Следовательно, на поверхности существует бесчисленное множество гиперболических точек, причем плошадь их сферического изображения отлична от нуля. На множестве гиперболических точек поверхности имеет место равенство полной внутренней и полной внешней кривизн. И так как внешняя кривизна заведомо отрицательна, то и внутренняя отрицательна. Но это невозможно, так как полная кривизна на любом множестве точек поверхности должна быть равна нулю. Таким образом, на поверхности с нулевой внутренней кривизной не может быть гиперболических точек.

Аналогично устанавливается, что на поверхности с нулевой внутренней кривизной не может быть эллиптических точек. Поэтому положительная и отрицательная внешние кривизны такой поверхности равны нулю. Поэтому верны:

ТЕОРема 1.2.6 (Погорелов [132]). Поверхность ограниченной внешней и нулевой внутренней кривизны развертывающаяся. Она имеет обычное для регулярных развертывающихся поверхностей строение с прямолинейными образующими и стационарной касательной плоскостью вдоль каждой образующей.

ТЕОРема 1.2.7 (Погорелов [132]). Полная поверхность класса $C^{1}$ с нулевой внутренней и ограниченной внешней кривизной - чилиндрическая.

Рассмотрим теперь поверхности, у которых положительная внутренняя кривизна отлична от нуля, а отрицательная равна нулю. На такой поверхности не может быть гиперболических точек. Доказывается это дословно такими же рассуждениями, как и в случае поверхностей нулевой кривизны.

Таким образом, поверхности неотрицательной внутренней кривизны являются также поверхностями неотрицательной внешней кривизны. Поэтому для них имеют место соответствующие теоремы [132; гл. $2, \S 4]$.

ТЕОРема 1.2 .8 (Погорелов [132]). Поверхность с неотричательной (не равной нулю) внутренней и ограниченной внешней кривизной с краем, лежащим в плоскости, есть выпуклая поверхность, т.е. является областью на границе выпуклого тела.

ТЕОРема 1.2.9 (Погорелов [132]). Полная поверхность с ограниченной внешней кривизной и неотрицательной внутренней кривизной (не равной нулю) есть либо замкнутая выпуклая поверхность, либо бесконечная выпуклая поверхность.

1.3. Аффинно-устойчивые погружения римановых метрик [40]. Другой подход к регулярности изометрических погружений, который гарантировал бы определенную связь между внутренними и внешне геометрическими характеристиками подмногообразия, был предложен С.З. Шефелем [152], [153], [155]. 
Свойство поверхности назовем геометрическим, если оно сохраняется при преобразованиях пространства $E^{n}$, принадлежаших некоторой группе $G$. Всегда предполагается, что группа $G$ содержит группу подобий и отлична от нее. Такие группы назьваются геометрическими. Классификация геометрических групп получена в [149], [150]. Отметим, что содержательным оказьвается только рассмотрение группы аффинных преобразований, псевдогруппы мёбиусовых преобразований (порожденных при $n>2$ подобиями и инверсиями) и, с несколько иными целями, - группы всех диффеоморфизмов фиксированной гладкости.

ОПРЕДЕЛЕНИЕ. Поверхность $F$ в $E^{n}$ назьвается $G$-устойчивым погружением метрики некоторого класса $\mathscr{H}$, если любое преобразование, принадлежашее группе $G$, переводит $F$ в поверхность, внутренняя метрика которой тоже принадлежит классу $\mathscr{H}$.

При этом подразумевается, что $G$ есть геометрическая группа (или псевдогруппа) преобразований в $E^{n}$. Поскольку тождественное преобразование id $\in G$, то очевидно, что внутренняя метрика самой поверхности $F$ принадлежит $\mathscr{H}$. В этом определении класс метрик $\mathscr{H}$ не обязательно исчерпьвается римановыми метриками. Соответственно, под погружением метрики здесь понимается $C^{0}$-гладкое (топологическое) погружение, являюшееся изометрией.

Существенно, что требование $G$-устойчивости поверхности не накладывает никаких априорных ограничений на размерность $n$ объемлющего пространства. Отметим, что $G$-устойчивые погружения метрик некоторого класса $\mathscr{H}$ (не исчерпываюшего всех допустимых метрик) всегда обладают некоторьм общим геометрическим свойством. Переход от любых погружений к $G$-устойчивьм позволяет установить двойственную связь между внешними и внутренними свойствами поверхностей.

Естественность понятия $G$-устойчивости иллюстрируют следуюшие утверждения, в более общей форме доказанные С.З. Шефелем в [147], [151], [152].

ТЕОРема 1.3 .1 (Шефель [151]). Аффинно устойчивыми погружсениями в $E^{n}$, $n \geqslant 3$, для класса двумерных римановых метрик положстельной кривизны являются только локально выпуклье поверхности в некотором $E^{3} \subset E^{n}$.

Класс же аффинно устойчивых погружений для двумерных римановых метрик отрицательной кривизны отнюдь не исчерпьвается поверхностями в $E^{3}$, но все такие погружения принадлежат классу так называемых седловых поверхностей, т.е. поверхностей, локально не допускающих строго опорных гиперплоскостей.

Теорема 1.3.2 (Шефель [152]). Пусть $G$ - группа диффеоморфизмов в $E^{n}$ гладкости $C^{\infty}$. Тогда G-устойчивыми погружениями для класса римановьх метрик гладкости $C^{l, \alpha}, l \geqslant 2,0<\alpha<1$, являются только поверхности той жее гладкости.

Наиболее привлекательной является ситуация, когда класс метрик $\mathscr{H}$, группа $G$ и класс поверхностей $\mathscr{M}$ находятся в следуюших отношениях.

1. Класс поверхностей $\mathscr{M}$ совпадает с классом $G$-устойчивых погружений метрик соответствуюшего ему класса метрик $\mathscr{H}$.

2. Каждая метрика класса $\mathscr{H}$ допускает погружение в виде поверхности класса $\mathscr{M}$. 
В этом случае класс поверхностей $\mathscr{M}$ и класс метрик $\mathscr{H}$ называются $G$-связанньl.мu.

1.4. Классы поверхностей и классы метрик. Классификация точек позволяет выделить шесть классов гладких поверхностей. Поверхности первых трех классов $M^{+}, M^{-}, M^{0}$ состоят, соответственно, только из эллиптических, гиперболических или параболических точек. Поверхности класса $M_{0}^{+}$состоят только из эллиптических и параболических, а поверхности класса $M_{0}^{-}$- только из гиперболических и параболических точек. Наконец, класс $M$ образуют все гладкие поверхности.

Поверхности класса $M_{0}^{+}$называются нормальнылми поверхностями неотрицательной кривизны, а поверхности классов $M_{0}^{-}, M^{-}$- соответственно, седловым ми и строго седловым.ми поверхностями.

ТеОРема 1.4 .1 (Шефель [152]). Класс $M^{+}$в $E^{n}$ состоит из лежащих в некотором $E^{3} \subset E^{n}$ локально выпуклых поверхностей. Полная поверхность класса $M^{+}$является полной выпуклой поверхностью (границей выпуклого тела в $E^{3}$ ). Нормальные поверхности неотрицательной кривизны (класс $M_{0}^{+}$) характеризуются тем, что либо каждая точка такой поверхности имеет окрестность в виде выпуклой поверхности, либо через эту точку проходит прямолинейная образующая с концами на границе поверхности, причем касательная плоскость вдоль этой прямолинейной образующей стационарна. Полная поверхность класса $M_{0}^{+}$является либо выпуклой поверхностью в $E^{3}$, либо чилиндром в $E^{n}$. Класс $M_{0}$ состоит из развертывающихся поверхностей. Полнье поверхности этого класса - иилиндры.

Седловые поверхности $F$ (класс $M_{0}^{-}$) могут быть охарактеризованы тем свойством, что никакая гиперплоскость не отсекает от $F$ әорбушку, т.е. область, замькание которой компактно и не выходит на гранищу $F$.

По знаку гауссовой кривизны естественно выделяются следующие классы двумерных римановых метрик: классы $K^{+}, K^{-}, K^{0}$ римановых метрик положительной, отрицательной и нулевой кривизны; классы $K_{0}^{+}, K_{0}^{-}$метрик неотрицательной и неположительной кривизны и класс $K$ всех римановых метрик. Классы поверхностей и метрик, отмеченные одинаковьми индексами, будем назьвать соответствуюшими.

Локальные свойства гладких поверхностей и метрик обычно сводятся к условиям на поверхность (или метрику) в каждой точке. Как правило, эти условия описьвают поведение поверхности (метрики) в окрестности точки с точностью до второго порядка малости. В дальнейшем геометрическое свойство поверхности будем назьвать локальньлм, если оно есть свойство точки поверхности и его выполнение в некоторой точке $p$ поверхности $F$ влечет выполнение этого же свойства в точке $p$ для любой другой поверхности, которая в окрестности точки $p$ совпадает с $F$ с точностью до бесконечно малых второго порядка.

Для классов поверхностей и метрик, выделенных на основе их локальных свойств, будем различать $G$-связанность в малом и $G$-связанность в целом и, соответственно, сформулируем две задачи: в малом и в целом.

Класс поверхностей $\mathscr{M}$ и класс метрик $\mathscr{H}$ назьваются $G$-связанными в малом, если: 1) класс поверхностей $\mathscr{M}$ совпадает с классом $G$-устойчивых погружений метрик класса $\mathscr{H}$ и 2 ) каждая метрика класса $\mathscr{H}$ допускает локальное погружение в виде 
поверхности класса $\mathscr{M}$. Задача в малом состоит в отыскании $G$-связанных в малом классов поверхностей и метрик.

Класс $\widetilde{\mathscr{M}}$ полных поверхностей и класс $\widetilde{\mathscr{H}}$ полньх метрик назьваются $G$-связанными в целом, если 1) класс поверхностей $\widetilde{\mathscr{M}}$ совпадает с классом $G$-устойчивых погружений метрик класса $\widetilde{\mathscr{H}}$ и 2 ) каждая метрика класса $\widetilde{\mathscr{H}}$ допускает погружение (в целом) в виде поверхности класса $\widetilde{\mathscr{M}}$. Задача в целом состоит в отыскании $G$-связанных в целом классов поверхностей и метрик.

В отличие от задачи в малом здесь даже в тех случаях, когда основой для выделения классов служат локальные свойства, на $G$-связанные в целом классы полных поверхностей и метрик как правило приходится налагать априорные условия не локального характера.

Основной вопрос о соответствии поверхностей и метрик в случае гладких поверхностей и для афффинной группы преобразований, как и в общем случае, состоит из задачи в малом и задачи в целом. Задача в малом для классов $K^{+}, K^{-}, K^{0}, K$ решена полностью; имеют место следующие две теоремы.

Tеорема 1.4 .2 ([40]). Kлассы $M^{+}, M^{-}, M^{0}, M$ гладких поверхностей и соответствующие им классы метрик попарно аффинно связаны в малом.

ТЕОРема 1.4 .3 ([40]). Если ограничиться теми классами гладких поверхностей, каждыи из которьх определяется локальным геометрическим свойством, то не существует аффинно связанных в малом пар, отличных от перечисленньл в в теореме 1.4 .2 и, бить может, парь $K_{0}^{-}, M_{0}^{-}$.

Теорема 1.4.2 объединяет в себе следующие утверждения.

1. Каждьй упомянутый класс поверхностей является афффинно инвариантньм.

2. Внутренняя метрика поверхности любого из этих классов принадлежит соответствующему классу метрик.

3. Аффиннно устойчивое погружение в $E^{n}$ метрики любого класса принадлежит соответствующему классу поверхностей.

4. Каждая метрика любого из классов допускает локальное погружение в виде поверхности соответствующего класса в $E^{n}$.

TEOРЕMA $1.4 .4([40])$. Классы $\widetilde{M}^{+}, \widetilde{M_{0}}, \widetilde{M}$ гладких полных односвязных поверхностей и соответствующие им классь римановых метрик аффинно связаны в иелом.

$C^{1}$-гладкая поверхность в $E^{3}$ назьвается нормальной поверхностью неотрищательной кривизны, если для каждой ее точки $x$ выполнено хотя бы одно из следующих утверждений: а) точка $x$ имеет окрестность в виде вьпуклой поверхности (в частности, эта окрестность может быть плоской областью); б) через точку $x$ проходит прямолинейная образуюшая, вдоль которой касательная плоскость стационарна.

Нормальной развертывающейся поверхностью назьвается такая нормальная поверхность неотрицательной кривизны, которая не имеет точек вьпуклости, т.е. является седловой.

Хорошо известно, что $C^{2}$-гладкая поверхность является нормальной поверхностью неотрицательной кривизны (нормальной развертывающейся поверхностью) тогда и только тогда, когда ее гауссова кривизна не отрицательна (равна нулю). 
Нетрудно видеть, что если точка нормальной поверхности неотрицательной кривизны не имеет окрестности в виде выпуклой поверхности, то проходящая через эту точку прямолинейная образуюшая единственна и никакая другая точка этой прямолинейной образуюшей не имеет такой окрестности, причем эта прямолинейная образующая продолжается до границы поверхности.

Нормальная поверхность неотрицательной кривизны по своей внутренней геометрии является многообразием неотрищательной кривизны, а нормальная развертьваюшаяся поверхность локально изометрична плоскости.

Полная нормальная поверхность неотрицательной кривизны, внутренняя кривизна которой не равна нулю, является вьпуклой поверхностью, а полная нормальная развертьваюшаяся поверхность является цилиндром. Каждая горбушка, отсекаемая от нормальной поверхности неотрицательной кривизны плоскостью, есть выпуклая поверхность.

Напомним, что через $K_{0}^{+}, K_{0}^{-}, K^{0}$ мы обозначили классы двумерных многообразий неотрицательной, неположительной и нулевой кривизны соответственно.

Теорема 1.4 .5 (Шефель [153]). Пусть $C^{1}$-гладкая поверхность $F$ в $E^{3}$ имеет конечную внешнюю положительную кривизну $\mu^{+}$. Если внутренняя метрика поверхности $F$ принадлежит одному из классов $K_{0}^{+}, K_{0}^{-}, K^{0}$, то сама поверхность $F$ является, соответственно, нормальной поверхностью неотрицательной кривизны, седловой поверхностью или нормальной развертывающейся поверхностью.

ТЕОРема 1.4.6 (Шефель [153], [154]). Если $C^{1}$-гладжая поверхность $F$ в $E^{3}$ имеет конечную внешнюю полоэительную кривизну и по своей внутренней геометрии принадлежит одному из классов $K_{0}^{+}, K_{0}^{-}, K^{0}$, то образ поверхности $F$ при аффинном преобразовании имеет внутреннюю метрику того же класса.

Иными словами, теорема утверждает, что $C^{1}$-гладкие поверхности ограниченной положительной внешней кривизны являются аффинно устойчивыми погружениями для метрик перечисленных в теореме классов.

Теорема 1.4 .7 (Шефель [153], [154]). Если $C^{1}$-гладкая поверхность $F$ является аффинно устойчивым погружением в $E^{3}$ метрики одного из классов $K_{0}^{+}, K_{0}^{-}$, $K$, то поверхность $F$ есть, соответственно, нормальная поверхность неотрицательной кривизнь, седловая поверхность, нормальная развертывающаяся поверхность.

Как следствие имеет место

Теорема 1.4 .8 (Шефель [153], [154]). $C^{1}$-гладкое изометрическое аффинно устойчивое погружение полной двумерной метрики нулевой кривизны в $E^{3}$ является чилиндром.

Аналогичный результат имеет место, если вместо $E^{3}$ объемлющим пространством будет $E^{n}(n \geqslant 3)$.

Глубокое изложение проблематики этой главы есть в обзоре Ю. Д. Бураго [40], первоначальная версия которого написана совместно с С. З. Шефелем. 


\section{Глава 2. Изометрические регулярные погружения двумерных метрик постоянной кривизны}

2.1. Классические результаты. Будем сначала рассматривать изометричные погружения в классе регулярных поверхностей класса $C^{r}(r \geqslant 2)$.

Имеет место

ТЕОРема 2.1.1. Полная связная регулярная поверхность постоянной гауссовой кривизны $K>0$ в евклидовом пространстве $E^{3}$ является стандартной сферой.

Эта теорема была сначала доказана Г. Минковским. Г. Либман дал ее доказательство в аналитическом случае, а потом Д. Гильберт предложил свое классическое доказательство.

Вместо регулярности поверхности можно потребовать лишш локальную вьпуклость поверхности или чтобы поверхность была гладкой поверхностью класса $C^{1}$ ограниченной внешней кривизны или $C^{1}$-гладким аффинно устойчивым погружением в классе метрик положительной кривизны. В первом случае из теоремы 1.2.9 следует, что поверхность есть замкнутая вьпуклая поверхность. Далее, из теоремы Погорелова об однозначной определенности общих замкнутых вьпуклых поверхностей [133] следует, что поверхность есть стандартная сфрера. Во втором случае из теоремы 1.4.7 следует вьпуклость поверхности, и дальше также используем теорему об однозначной определенности. Тогда утверждение теоремы будет следовать из результатов А. В. Погорелова [131]-[133] и С. З. Шефеля [153], [154], сформулированных в предыдущей главе.

ТЕОРема 2.1.2. Полная связная регулярная поверхность нулевой гауссовой кривизны в евклидовом пространстве $E^{3}$ является иилиндром.

Условие регулярности можно ослабить, потребовав от поверхности лишш принадлежность к классу гладких поверхностей ограниченной внешней кривизны [132]. Верность этой теоремы в классе гладких поверхностей ограниченной внешней кривизны утверждает теорема 1.2 .7 ; в классе $C^{1}$-гладких, аффинно устойчивых погружений метрик нулевой кривизны это есть теорема 1.4.8. Можно ослабить и это условие регулярности, заменив его условием принадлежности прямой объемлющего пространства поверхности. Справедлива

Лемма 2.1 .1 ([29]). Пусть $F-$ двумерная класса $C^{0}$ поверхность в евклидовом пространстве $E^{n}$, изометричная плоскости, и на $F$ лежит прямая $p$ обгемлющего пространства. Тогда F будет иилиндром с образующей, параллельной прямой $р$.

ДокАЗАТЕльство. Пусть $\bar{F}$ - плоскость, изометричная поверхности $F ; \bar{Q} \in \bar{F}$ точка, соответствующая $Q \in F$ по изометрии; $\bar{P} \bar{Q}$ - расстояние на $\bar{F}$ между точками $\bar{P}, \bar{Q} ; P Q-$ расстояние между точками $P, Q$ в $E^{n}$.

Введем на $\bar{p}$ в качестве параметра длину $s$, отсчитываемую от фиксированной точки $\bar{P}(0) \in \bar{p} . \quad M(s)-$ кратчайшая, перпендикулярная $\bar{p}$, которая проходит через точку $\bar{P}(s) \in \bar{p}$. 
Теперь через каждую точку $P(s) \in p$ поверхности $F$ проведем гиперплоскость $E^{n-1}(s)$, ортогональную $p, F(s)=E^{n-1}(s) \cap F$. Покажем, что для любого $s F(s)=$ $M(s)$. Допустим, что для какого-то $s_{0}$ в $F\left(s_{0}\right)$ будет содержаться точка $Q \in M\left(s_{1}\right)$, где $s_{1} \neq s_{0}$. Возьмем на $\bar{F}$ треугольник $\bar{P}\left(s_{0}\right) \bar{Q} \bar{P}(s)$. Так как $\bar{Q} \notin \bar{M}\left(s_{0}\right)$, то кратчайшая $\bar{P}\left(s_{0}\right) \bar{Q}$ не перпендикулярна к $\bar{p}$. Точку $\bar{P}(s)$ будем выбирать на луче, который образует с $\bar{P}\left(s_{0}\right) \bar{Q}$ острый угол.

При достаточно большом $s$ угол $\bar{P}\left(s_{0}\right) \bar{Q} \bar{P}(s)$ будет тупьм и

$$
\bar{P}\left(s_{0}\right) \bar{P}(s)>\bar{Q} \bar{P}(s) .
$$

Рассмотрим треугольник $P\left(s_{0}\right) Q P(s)$. Он прямоугольньй с прямым углом $Q P\left(s_{0}\right) P(s)$.

Значит,

$$
P(s) Q>P\left(s_{0}\right) P(s)
$$

HO

$$
P\left(s_{0}\right) P(s)=\bar{P}\left(s_{0}\right) \bar{P}(s), \quad Q P(s) \leqslant \bar{Q} \bar{P}(s) .
$$

Отсюда $\bar{P}(s) \bar{Q}>\bar{P}\left(s_{0}\right) \bar{P}(s)$. В результате приходим к противоречию, т.е.

$$
F(s)=M(s) \text {. }
$$

Пусть $\bar{Q}$ - произвольная точка $\bar{F} ; \bar{q}$ - прямая, проходящая через $\bar{Q}$ и параллельная $\bar{p}$.

Покажем, что $q$ будет прямой евклидова пространства, параллельной $p$. Пусть $\bar{Q}_{1}, \bar{Q}_{2}-$ произвольные точки на $\bar{q}$. Опустим из $\bar{Q}_{1}, \bar{Q}_{2}$ перпендикуляры на $\bar{p} . \bar{P}_{1}, \bar{P}_{2}-$ основания перпендикуляров, $\bar{P}_{1} \bar{P}_{2}=\bar{Q}_{1} \bar{Q}_{2}$.

Рассмотрим пространственньй четырехугольник $P_{1} P_{2} Q_{2} Q_{1}$. Натянем на этот четырехугольник евклидово пространство $E^{3}$. Началом прямоугольной системы координат в $E^{3}$ возьмем $P_{1}$, ось $z$ направим по прямой $p$. Точки $P_{1}, P_{2}, Q_{1}, Q_{2}$ имеют следуюшие координаты:

$$
\begin{gathered}
P_{1}(0,0,0), \quad P_{2}\left(0,0, z_{2}\right), \quad Q_{1}\left(x_{1}, y_{1}, 0\right), \quad Q_{2}\left(x_{2}, y_{2}, z_{2}\right), \\
P_{1} P_{2}=\left|z_{2}\right|, \quad Q_{1} Q_{2}=\sqrt{\left(x_{1}-x_{2}\right)^{2}+\left(y_{1}-y_{2}\right)^{2}+z_{2}^{2}} \geqslant P_{1} P_{2},
\end{gathered}
$$

но $P_{1} P_{2}=\bar{P}_{1} \bar{P}_{2}, Q_{1} Q_{2} \leqslant \bar{Q}_{1} \bar{Q}_{2}$ и, значит, $Q_{1} Q_{2} \leqslant P_{1} P_{2}$

Отсюда $Q_{1} Q_{2}=P_{1} P_{2}$. А это возможно, если $x_{1}=x_{2}, y_{1}=y_{2}$, т.е. отрезок $Q_{1} Q_{2}$ будет параллелен $p$ и он будет кратчайшей на $\bar{F}$.

Ввиду произвольности точек $\bar{Q}_{1}, \bar{Q}_{2}, \bar{Q}$ утверждение леммы доказано [29], [36].

Еще в конце $30-$ х годов XIX века $\Phi$. Миндинг исследовал некоторые свойства поверхностей вращения, имеющих гауссову кривизну $K=$ const $<0$. Одна из этих поверхностей (псевдосфера) была более детально изучена Э. Бельтрами. На этих поверхностях были особые линии и точки, где нарушалась регулярность поверхности. Первым поставил вопрос о возможности реализовать плоскость Лобачевского в евклидовом пространстве $E^{3}$ поверхностью без особенностей Д. Гильберт. Он дал отрицательньй ответ на этот вопрос. 
ТеОРема 2.1.3 (Гильберт [88]). В евклидовом пространстве $E^{3}$ не существует аналитической полной поверхности постоянной отрицательной кривизнь.

Дополнительные исследования [105], [83] показьвают, что справедлива

Tеорема 2.1.4 ([141]). В $E^{3}$ не существует $C^{2}$-гладжой поверхности, изометричной в иелом плоскости Лобачевского.

Если ограничиться предположением гладкости поверхности лишш класса $C^{1}$, то из результатов Н. Кейпера следует возможность изометрического погружения плоскости Лобачевского в $E^{3}$ в этом классе поверхностей.

Допускает ли теорема 2.1.4 обобщение на класс гладких поверхностей класса $C^{1}$ локально ограниченной внешней кривизны, неизвестно [141].

Пусть $M^{3}$ - полное односвязное риманово многообразие постоянной секционной кривизны $K_{0}$. Для регулярной поверхности $F \subset M^{3}$ определяются внутренняя кривизна (гауссова кривизна) $K_{\text {int }}$ и внешняя кривизна $K_{\text {ext }}$, как произведение главньх кривизн. Из уравнения Гаусса следует, что

$$
K_{\mathrm{int}}=K_{\mathrm{ext}}+K_{0} .
$$

Если $K_{0}=+1$, то $M^{3}$ есть стандартная сффера $S^{3}$, если $K_{0}=-1$, то $M^{3}$ есть гиперболическое пространство $H^{3}$.

Для пространств постоянной кривизны справедливы

ТеОРема 2.1.5 ([159]). Полные регулярные класса $C^{2}$ поверхности в $S^{3}$ и $H^{3}$ c постоянной гауссовой кривизной $K_{\mathrm{int}}>0$ и постоянной $K_{\mathrm{ext}} \geqslant 0$ являются омбилическими поверхностями.

Теорема 2.1.6 ([159]). Не существует полной регулярной класса $C^{2}$ поверхности в $S^{3}$ и $H^{3}$ с постоянными $K_{\mathrm{int}}<0$ и $K_{\mathrm{ext}}<0$.

Для сфферического пространства $S^{3}$ мы немедленно получаем: если $K_{\text {ext }}>0$, то поверхность есть малая сфера; если $K_{\text {ext }}=0$, то поверхность есть вполне геодезическая большая сфера $S^{3}$; если $-1<K_{\text {ext }}<0$, то полной поверхности в $S^{3}$ не существует. Действительно, в этом случае $K_{\text {int }}>0$ и универсальная накрьвающая поверхности гомеоморфна сфере. С другой стороны, $K_{\text {ext }}<0$, и поэтому главные кривизны разного знака. Мы имеем на сфере $S^{2}$ непрерьвное поле направлений, что невозможно, так как эйлерова характеристика сфферы отлична от нуля. Если $K_{\text {ext }}<-1$, то из теоремы 2.1.6 следует, что поверхности не существует.

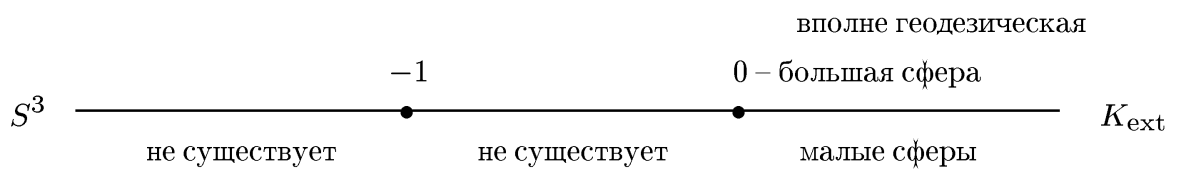

2.2. Плоские метрики в сфере. Остается случай, когда $K_{\text {ext }}=-1, K_{\text {int }}=0$. Существует много полных плоских регулярных поверхностей в сферическом пространстве $S^{3}$. Из классической теоремы Бельтрами-Эннепера следует, что кручение асимптотических линий на поверхности постоянной кривизны постоянно, но противоположного знака для различных семейств. Аналитические линии одного семейства получаются друг из друга клиффордовым сдвигом, т.е. изометрией $A: S^{3} \rightarrow S^{3}$ такой, что расстояние на сфере $d(x, A(x))$ постоянно [159], [160]. 
Опишем все плоские торы в $S^{3}$. Будем рассматривать сферу $S^{2}$ как единичные мнимые кватернионы, сферу $S^{3}$ как единичные кватернионы. Тогда расслоение Хопфа:

$$
h: S^{3} \rightarrow S^{2}
$$

имеет вид

$$
h(q)=\bar{q} i q, \quad q \in S^{3},
$$

где $i$ - мнимая единица, $i^{2}=-1$.

Рассмотрим $S^{1}$ как множество единичных комплексных чисел и $c: S^{1} \rightarrow S^{2}$ погружение класса $C^{2}$. Так как $S^{2} \subset E^{3}$, то мы можем определить индикатрису касательных $c^{1}: S^{1} \rightarrow S^{2}$. Рассмотрим $T=h^{-1}\left[c\left(S^{1}\right)\right]-$ тор Хопфа, частный случай плоского тора в $S^{3}$, возможно с самопересечениями [130]. На $T$ имеется два семейства асимптотических линий, одно из которых есть семейство больших окружностей, второе семейство асимптотических линий, которые также являются замкнутыми кривьди.

Пусть $\gamma: S^{1} \rightarrow S^{3}$ есть регулярная параметризация любой из этих асимптотических линий. Ясно, что $h: \gamma\left(S^{1}\right) \rightarrow c\left(S^{1}\right)$ есть накрытие. Мы дадим другое описание асимптотических линий $\gamma$ относительно $c$. Пусть $U S^{2}$ есть расслоение единичных векторов над $S^{2}, S O(3)$ - группа собственных ортогональных преобразований в $E^{3}$.

По отображению $c: S^{1} \rightarrow S^{2}$ мы определим $\widehat{c}: S^{1} \rightarrow S O(3)$ как $\widehat{c}=\left(c, c^{\prime}, c c^{\prime}\right)$.

Определим $\pi: S^{3} \rightarrow S O(3)$ как

$$
\pi(q)=(\widetilde{q} i q, \widetilde{q} j q, \widetilde{q} k q)
$$

Ясно, что $\pi$ есть двойное накрытие и $\pi(q)=\pi(-q)$ для любого $q$. Если $\rho_{\theta}: U S^{2} \rightarrow$ $U S^{n}$ есть вращение на угол $\theta$ любого слоя $U S^{2}$, то для любого $\gamma$ существует $\theta$ такое, что

$$
\pi\left(\gamma\left(S^{1}\right)\right)=\rho_{\theta}\left(\widehat{c}\left(S^{1}\right)\right)
$$

Напомним, что каждая регулярная класса $C^{1}$ кривая $c: S^{1} \rightarrow S^{2}$ регулярно гомотопна к окружности, пробегаемой один раз $(c \sim 0)$, окружности, пробегаемой $m$ раз $(c \sim$ $m-1)$.

Если $c \sim 0$, то $\pi: \gamma\left(S^{1}\right) \rightarrow \rho_{\theta} \circ \widehat{c}\left(S^{1}\right)$ есть двойное накрытие. Если $c \sim 1$, то $\pi: \gamma\left(S^{1}\right) \rightarrow \rho_{\theta} \circ \widehat{c}\left(S^{1}\right)$ есть диффеоморфизм.

Выберем $C^{2}$-погружения $a, b: S^{1} \rightarrow S^{2}$. Пусть $\alpha, \beta: S^{1} \rightarrow S^{3}$ - замкнутые асимптотические линии соответствующих торов Хопфа. Определим

$$
\bar{\alpha} \cdot \beta: S^{1} \times S^{1} \rightarrow S^{3}
$$

как

$$
\bar{\alpha} \cdot \beta(s, t)=\bar{\alpha}(s) \cdot \beta(t), \quad(s, t) \in S^{1} \times S^{1} .
$$

Пусть $k_{c}: S^{1} \rightarrow \mathbb{R}$ есть геодезическая кривизна кривой $c: S^{1} \rightarrow S^{2}$. 
Tеорема 2.2.1 ([159]). Пусть $a, b: S^{1} \rightarrow S^{2}$ суть $C^{2}$-погружения. Eсли $k_{a}\left(S^{1}\right) \cap$ $k_{b}\left(S^{1}\right)=\varnothing$, то $\bar{\alpha} \cdot \beta$ есть погружение $с$ плоской метрикой. И с точностью до движения в $S^{3}$ все погруженные плоские торы получаются таким способом.

Можно показать, что если $a \sim 0, b \sim 0$, то $\bar{\alpha} \cdot \beta$ есть двойное накрытие их образа, и образ имеет центральную симметрию [172].

Д. Вайнер полностью определил плоские погруженные торы в $S^{3}$ их грассмановым образом.

Пусть $\alpha$ - регулярная замкнутая кривая на стандартной сфере $S^{2}$. Пусть $d s$ и $k-$ элемент длины дуги и геодезическая кривизна кривой $\alpha$. Тогда определим

$$
\mu(\alpha)=\max \left\{\int_{\beta} k d s: \beta-\text { дуга кривой } \alpha\right\}, \quad \tau(\alpha)=\int_{\alpha} k d s .
$$

Если $\gamma$ есть индикатриса касательных регулярной замкнутой кривой в $S^{2}$, то $\mu(\gamma)<\pi$.

Пусть $X: T \rightarrow S^{3}$ есть плоский тор в $S^{3}$. Рассматривая его как поверхность в $E^{4}$, мы получим грассманов образ $T$ в грассманово многообразие $G(2,4)$,

$$
G: T \rightarrow G(2,4)
$$

$G(2, n)=S_{1} \times S_{2}$ есть метрическое произведение двух стандартных сфер радиуса $\frac{1}{\sqrt{2}}$. Мы говорим, что поверхность в $G(2, n)$ удовлетворяет условию Е, если $M=\gamma_{1} \times \gamma_{2}$, где $\gamma_{i}$ суть замкнутые кривые в $S_{i}(i=1,2)$, для которых $\tau\left(\gamma_{i}\right)=0$ и $\mu\left(\gamma_{1}\right)+\mu\left(\gamma_{2}\right)<\pi$.

Пусть $\left[\gamma_{i}\right]$ обозначает гомотопический класс в $\pi_{1}\left(\gamma_{1} \times \gamma_{2}\right)$ параметризации $\gamma_{i}$. Пусть $H_{11}$ - подгруппа в $\pi\left(\gamma_{1} \times \gamma_{2}\right)$, порожденная $\left\{\left[\gamma_{1}\right]+\left[\gamma_{2}\right],\left[\gamma_{1}\right]-\left[\gamma_{2}\right]\right\}, H_{12}-$ подгруппа, порожденная $\left\{2\left[\gamma_{1}\right],\left[\gamma_{2}\right]\right\}, H_{22}=\pi_{1}\left[\gamma_{1} \times \gamma_{2}\right], H_{m n}=\left\{m\left[\gamma_{1}\right], n\left[\gamma_{2}\right]\right\}$.

Tеорема 2.2.2 (Вайнер [172]). Пусть $G: T \rightarrow G(2,4)$ есть гладкое отображсение тора в грассманово многообразие $G(2,4)$. Тогда $G$ есть грассманов образ плоского тора $X: T \rightarrow S^{3} \subset E^{4}$ тогда и только тогда, когда $G(T)$ удовлетворяет условию $\mathrm{E}, G \rightarrow \gamma_{1} \times \gamma_{2}$ есть накрытие и $G_{*}\left(\pi_{1}(T)\right) \subset H_{m n}$, если $\gamma_{1} \sim m$, $\gamma_{2} \sim n$.

В случае, когда $\mu\left(\gamma_{1}\right)<\frac{\pi}{2}, \mu\left(\gamma_{2}\right)<\frac{\pi}{2}$, достаточное условие было доказано в [65]. Из класса плоских торов торы Хопфа выделяются условием, что их грассманов образ $\gamma_{1} \times \gamma_{2}$ таков, что кривая $\gamma_{1}$ есть большая окружность.

Из теоремы 2.2.2 следует, что нет плоской бутылки Клейна в стандартном проективном пространстве $P^{3}$ с метрикой постоянной кривизны [172].

ОпРЕДЕлЕниЕ. 1. Тор Клиффорда есть риманово многообразие, изометричное $E^{2} / \Gamma$, где Г есть ортогональная решетка диаметра $\pi$.

2. Произведение окружностей в $S^{3}$ есть прямое произведение двух окружностей в $E^{4}$ с центром в начале координат, расположенных в ортогональных плоскостях. Если $r_{1}, r_{2}$ - радиусы этих окружностей, то $r_{1}^{2}+r_{2}^{2}=1$.

Ясно, что произведение окружностей есть тор Клиффорда. Верно и в некотором смысле обратное. 
Теорема 2.2.3 (Вайнер [173]). Пусть $X: T \rightarrow S^{2}$ есть изометрическое погружение плоского тора с диаметром $\leqslant \pi$. Eсли $X(T)$ содерэит пару диаметрально противоположных точек, то $X(T)$ - произведение окружностей.

Из теоремы 2.2.3 следует

СлЕДСТВИЕ 2.2 .1 ([173]). Вложенный тор Клиффорда является произведением окружностей.

СлЕДСТВИЕ 2.2.2. Нет других вложенных плоских торов с диаметром $\leqslant \pi$ в $S^{3}$, кроме торов Клиффорда.

В [104] изучались внешне геометрические свойства плоских торов. Была доказана

Теорема 2.2.4 (Китагава [104]). Ecли $f: M \rightarrow S^{3}$ есть изометрическое вложение плоского тора $M$ в сферическое пространство $S^{3}$, то образ $f(M)$ инвариантен относительно иентральной симметрии в $S^{3}$.

В теореме нельзя заменить вложенную поверхность на погруженную, так как существует пример погруженного плоского тора, не инвариантного при центральной симметрии. Однако остается открытьм вопрос: для каждого ли погруженного плоского тора сушествует пара диаметрально противоположных точек $S^{3}$, которые принадлежат поверхности [104]?

Для случая $K_{\text {ext }}=0$ имеет место аналог леммы 2.1.1.

Теорема 2.2.5 ([30], [36]). Пусть $F$ - гладкая класса $C^{1}$ поверхность в $S^{3}(1)$, изометричная сфере $S^{2}(1)$. Если на $F$ лежит большая окружность облемлющей сферь $S^{3}$, то $F$ является вполне геодезической большой сферой.

ДокАЗАТЕЛЬство. Пусть $S^{1}-$ большая окружность $S^{3}$, которая лежит на $F$; точки $O_{1}, O_{2} \in F$, полярные $S^{1} ; \rho(x, y)$ - расстояние на поверхности $F ; r$ - расстояние в $S^{3}$. Возьмем на $S^{1}$ две диаметрально противоположные точки $X, Y$. Тогда

$$
\begin{aligned}
\rho\left(X, O_{1}\right)=\frac{\pi}{2}, \quad \rho\left(Y, O_{1}\right) & =\frac{\pi}{2} ; \quad r\left(X, O_{1}\right)=r_{1}, \quad r\left(Y, O_{1}\right)=r_{2} ; \\
r_{1} \leqslant \frac{\pi}{2}, & r_{2} \leqslant \frac{\pi}{2} .
\end{aligned}
$$

Но $r_{1}+r_{2}=\pi$, следовательно, $r_{1}=\frac{\pi}{2}, r_{2}=\frac{\pi}{2}$. Проведем сфреру $S^{2}\left(O_{1}\right)$, содержашую $S^{1}$ и $O_{1}$. Все кратчайшие $X O_{1}$, где $X \in S^{1}$, сферы $S^{2}\left(O_{1}\right)$ лежат на поверхности $F$, а значит, полусфера $S^{2}\left(O_{1}\right)$, которая содержит $O_{1}$, лежит на $F$. Часть поверхности $F$, которая содержит $O_{2}$, также будет полусферой. Но так как $F$ класса $C^{1}$, то $S^{2}\left(O_{1}\right)=$ $S^{2}\left(O_{2}\right)$ и $F$ будет вполне геодезической большой сфферой.

Для гиперболического пространства $H^{3}$

$$
K_{\mathrm{int}}=K_{\mathrm{ext}}-1
$$

Если $K_{\text {ext }}>1$, то полная регулярная поверхность в $H^{3}$ есть сфера. Если $K_{\text {ext }}<0$, то такой поверхности не существует. Остается сегмент $0 \leqslant K_{\text {ext }} \leqslant 1$. Для $K_{\text {ext }}=1$, $K_{\mathrm{int}}=0$ полная регулярная поверхность в $H^{3}$ есть либо орисфера, либо эквидистанта геодезической (Ю.А. Волков, С.М.Владимирова [170]). 
Есть много полных поверхностей в $H^{3}$ с $K_{\text {ext }}=0$. Они могут быть сколь угодно высокого рода.

Изометрические погружения плоскости Лобачевского $H^{2}(-1)$ в $H^{3}(-1)$ изучались К. Номидзу [125]. Эти поверхности являются 1-сильно параболическими. Кривая, ортогональная к полным вполне геодезическим слоям, имеет кривизну не больше 1 , где кривизна взята в метрике поверхности.

В [125] явно предъявлено однопараметрическое семейство изометрических погружений $H^{2}(-1)$ в $H^{3}(-1)$. В [92] описание изометрических погружений $H^{2}(-1)$ в $H^{-3}(-1)$ трансформировалось в решение вырожденного уравнения Монжа-Ампера в единичном круге.

Каждому регулярному изометрическому погружению $H^{2}(-1)$ в $H^{3}(-1)$ соответствует решение уравнения Монжа-Ампера

$$
\left(\frac{\partial^{2} u}{\partial \xi_{i} \partial \xi_{j}}\right)=0, \quad \xi=\left(\xi_{1}, \xi_{2}\right) \in D
$$

Обратно, каждому регулярному решению $U$ уравнения (2.2.1) отвечает регулярное изометрическое погружение $H^{2}(-1)$ в $H^{3}(-1)$ с первой квадратичной формой

$$
g_{i j}=\lambda^{-1}\left(\lambda^{2} \delta_{i j} \xi_{i} \xi_{j}\right), \quad \lambda=\sqrt{1-\xi_{i}^{2}-\xi_{2}^{2}}
$$

и второй квадратичной формой (см. [92])

$$
h_{i j}=\lambda^{-1} \frac{\partial^{2} u}{\partial \xi_{i} \partial \xi_{j}}, \quad i, j=1,2 .
$$

Аналогичная задача решена для изометрическогопогружения $H^{2}(-1)$ в $H^{3}(c)$ [91].

Каждому изометрическому погружению $H^{2}(-1)$ в $H^{3}(c)$ соответствует решение уравнения Монжа-Ампера

$$
\operatorname{det}\left(\frac{\partial^{2} u}{\partial \zeta_{i} \partial \zeta_{j}}\right)=\frac{-c-1}{\left(1-\zeta_{1}^{2}-\zeta_{2}^{2}\right)^{2}}
$$

в некоторой области $D$.

И обратно. Каждому гладкому решению (2.2.2) соответствует гладкое изометрическое погружение $H^{2}(-1)$ в $H^{3}(c)$ со следуюшими первой и второй квадратичными формами $g, h$ :

$$
\begin{aligned}
& g_{i j}=\lambda^{-4}\left(\lambda^{2} \delta_{i j}+\zeta_{i} \zeta_{j}\right), \quad \lambda=\sqrt{1-\zeta_{1}^{2}-\zeta_{2}^{2}}, \\
& h_{i j}=\lambda^{-1} \frac{\partial^{2} u}{\partial \zeta_{i} \partial \zeta_{j}}, \quad i, j=1,2 .
\end{aligned}
$$

Если $0<K_{\text {ext }}<1$, то известны эквидистанты и поверхности врашения. Неизвестно, существуют ли другие полные поверхности.

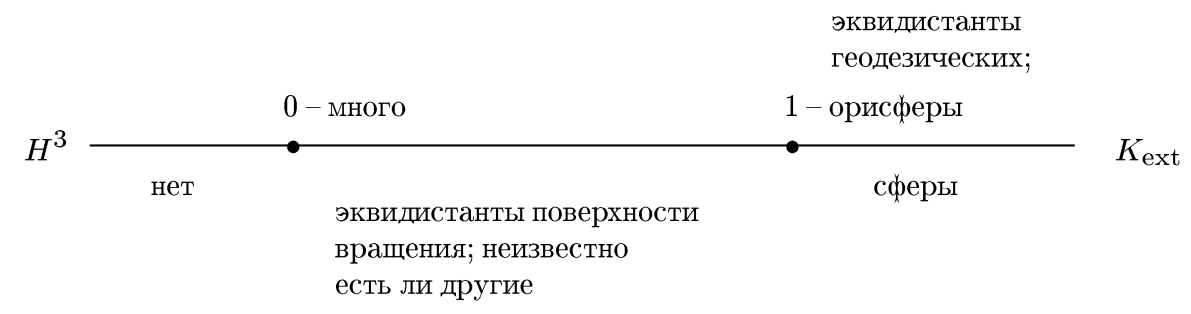


2.3. Изометрические погружения двумерных плоских метрик. Тонкие результаты по погружению локально евклидовых метрик получены И.Х. Сабитовым [144].

ТЕОРема 2.3.1 (Сабитов [144]). Если область с локально евклидовой метрикой допускает изометрическое погружение в $E^{2}$, то она изометрически вложима в $E^{3}$. Всякая односвязная область с локально евклидовой метрикой изометрически вкладывается в $E^{3}$.

Пусть в плоской области $D$ (односвязной или многосвязной) задана локально евклидова метрика

$$
d s^{2}=E(u, v) d u^{2}+2 F(u, v) d u d v+G(u, v) d v^{2}, \quad(u, v) \in D
$$

Локальная евклидовость метрики (2.3.1) означает, что каждая точка из $D$ имеет окрестность, изометричную некоторому кругу на плоскости $E^{2}$ со стандартной евклидовой метрикой $d x^{2}+d y^{2}$.

Таким образом, локальная изометрическая вложимость локально евклидовой метрики (2.3.1) в евклидову плоскость $E^{2}$ существует по самому определению локально евклидовой метрики. Нас будет интересовать вопрос об изометрических вложениях и погружениях в $E^{2}$ “в целом" всей области $D$, или, по-другому, вопрос о существовании отображения

$$
x=x(u, v), \quad y=y(u, v),
$$

приводящего (2.3.1) в целом к виду $d x^{2}+d y^{2}$. При этом отображении формулы $(2.3 .2)$ дают изометрическое погружение $D$ в $E^{2}$, если оно однозначно, и вложение, если оно однолистно во всей области $D$.

Пусть в круговой $n$-связной области $D_{n}$ (т.е. в круге - при $n=1$ и в ограниченной $n$ окружностями области - при $n \geqslant 2)$ плоскости переменных $(\xi, \eta)$ задана локально евклидова метрика $d s^{2}$. Без нарушения обшности можем считать, что координаты $(\xi, \eta)$ являются изотермическими (общий случай приводится к этому некоторым квазиконформным преобразованием). Тогда

$$
d s^{2}=\Lambda^{2}(\xi, \eta)\left(d \xi^{2}+d \eta^{2}\right), \quad \Lambda>0, \quad \Delta \ln \Lambda=0
$$

Предположим, далее, что регулярность $\Lambda(\xi, \eta)$ в замкнутой области $\bar{D}_{n}$ такова, что единственное (с точностью до движения в $E^{2}$ ) отображение $z=x+i y=\Phi(\zeta)$, $\zeta=\xi+i \eta$, приводящее (2.3.3) к стандартному виду $d s^{2}=d x^{2}+d y^{2}$, имеет в $\bar{D}_{n}$ гладкость класса $C^{m}, m \geqslant 1$. Напомним, что отображение $\Phi(\zeta)$ определяется через $\Lambda(\eta)$ равенством $\left|\Phi^{\prime}(\eta)\right|=\Lambda(\eta)$; следовательно, если $\Lambda \in C^{m, \alpha}, m \geqslant 0,0<\alpha<1$, то $\Phi(\eta) \in C^{m+1, \alpha}$.

Всякое условие однозначности голоморфной функции $z=\Phi(\zeta)$ дает нам изометрическую погружаемость метрики (2.3.3) в $E^{2}$, а всякое условие ее однолистности дает вложимость $(2.3 .3)$ в $E^{2}$ - и обратно. Справедлива 
Теорема 2.3.2 (Сабитов [144]). Локально евклидова метрика (2.3.1) гладкосmи $C^{m, \alpha}, m>0,0<\alpha \leqslant 1$, определенная в односвязной области $D$ с границей гладкости $C^{m+1, \alpha}$, изометрически погружается в $E^{2}$ отображением гладкос$m u C^{m+1, \alpha}$.

Рассмотрим более подробно вопрос о вложениях метрики $(2.3 .3)$ в $E^{2}$, считая $(2.3 .3)$ заданной в круге $\bar{\Omega}: \xi^{2}+\eta^{2} \leqslant 1$.

Пусть поворот любой дуги границы круга $D$ в метрике (2.3.3) неотрицателен. Тогда $D$ вкладьвается в $E^{2}$ в виде вьпуклой области.

Это - следствие теоремы о реализации в виде шапочки каждой выпуклой области на многообразии неотрицательной кривизны.

Изометрические вложения (2.3.3) в $E^{2}$ описьвает следующая

Теорема 2.3.3 (Сабитов [144]). Для того чтобы локально евклидова метрика (2.3.3), заданная в многосвязной области $D_{n}$, допускала изометрическое вложение в $E^{2}$, необходимо и достаточно, чтобь погружение $z=\Phi(\zeta)$ было однолистным на каждой из окружностей $\Gamma_{j}, 0 \leqslant j \leqslant n$.

В [146] для заданных в односвязной области метрик даются явные формулы для погружений и приводится ряд достаточных условий для существования изометрических вложений. В случае многосвязных областей задания метрик даются необходимые условия существования изометрических погружений и приводится классификация случаев, когда метрика не допускает изометрического погружения в евклидову плоскость.

Если область двусвязна, то она изометрически погружается в $E^{3}$.

Теорема 2.3.4 (Сабитов [143]). Пусть отображение $z=\Phi(\eta)$ является в $\bar{D}_{n}$ однозначным (т.е. $\bar{D}_{n}$ с метрикой (2.3.1) изометрически погружсется в $E^{2}$ ). Тогда область $\bar{D}_{n}$ с метрикой (2.3.1) реализуется в $E^{3}$ в виде развертывающейся поверхности гладкости $C^{\infty}$ в $D_{n}$ и гладкости $C^{m}$ в $\bar{D}_{n}$.

СЛЕДСТВИЕ. Круг $\bar{D}_{1}$ с локально евклидовой метрикой (2.3.1) допускает изометрическое вложсние в $E^{3}$ в виде развертывающейся поверхности.

Теорема 2.3 .5 (Сабитов [143]). Пусть в области $\bar{D}_{2}$ (т.е. в кончентрическом кольце) с локально евклидовой метрикой (2.3.3) отображсние $z=\Phi(\eta)$ неоднозначно. Тогда $\bar{D}_{2}$ можсно изометрически погрузить в $E^{3}$ в виде развертывающейся поверхности гладкости $\mathrm{C}^{m}$.

Существует пример локально евклидовой метрики, заданной в трехсвязной области и не погружаемой в $E^{3}$ в виде $C^{1}$-гладкой поверхности с ограниченной в смысле А. В. Погорелова внешней кривизной [143]. В [169] дано явное представление решений уравнения

$$
z_{x x} z_{y y}-z_{x y}^{2}=0
$$

с условием $z_{x x} \neq 0$. Это дает явное представление поверхностей (без точек уплощения) нулевой гауссовой кривизны в трехмерном евклидовом пространстве.

Как известно, имеется пять топологически различных типов локально евклидовых двумерных полных многообразий: плоскость, цилиндр, тор, лист Мёбиуса и бутылка Клейна [134]. 
Из этих многообразий регулярно изометрически погружаются в $E^{3}$ только плоскость и цилиндр. Элементарно погружается в $E^{4}$ тор с евклидовой метрикой. Параметрические уравнения тора с локально евклидовой метрикой $d s^{2}=d u^{2}+d v^{2}$ в $E^{4}$ имеют следуюший вид: $x_{1}=\cos u, x_{2}=\sin u, x_{3}=\cos v, x_{4}=\sin v$. Изометрическое погружение в $E^{4}$ бутылки Клейна было построено С. Томпкинсом [165] (другой пример погружения бутылки Клейна был построен А. Б. Ивановым [93]). Д. Бланушей [22] было построено погружение листа Мёбиуса в $E^{4}$.

Предложенное Томпкинсом погружение в $E^{4}$ бутылки Клейна определяется следуюшими параметрическими уравнениями:

$$
\begin{aligned}
x_{1} & =\cos u \cos v, & x_{2} & =\sin u \cos v, \\
x_{3} & =2 \cos \frac{u}{2} \sin v, & x_{4} & =2 \sin \frac{u}{2} \sin v .
\end{aligned}
$$

Эта поверхность имеет линию самопересечения (окружность $x_{1}^{2}+x_{2}^{2}=1, x_{3}=x_{4}=0$ ). Если ввести параметры

$$
U=u, \quad V=\int \sqrt{1+3 \cos ^{2} v} d v
$$

то внутренняя метрика погружения определится линейнњм элементом $d s^{2}=d U^{2}+$ $d V^{2}$, т.е. является локально евклидовой.

Погружение бутылки Клейна, предложенное А. Б. Ивановьм, определяется следуюшими параметрическими уравнениями:

$$
x_{1}=\cos u, \quad x_{2}=\sin u, \quad x_{3}=a \sin \left(v+\frac{u}{2}\right), \quad x_{4}=b \sin 2\left(v+\frac{u}{2}\right) .
$$

Представление о полученной поверхности может быть получено следуюшим образом. При фиксированном значении $u$ получается лемниската Бернулли, расположенная в плоскости, параллельной $O x_{3} x_{4}$ и проходящей через точку $(\cos u, \sin u, 0,0)$. Когда $u$ изменяется от 0 до $2 \pi$, эта лемниската с заданным направлением обхода совершает поворот на $\pi$ и совмешается с лемнискатой, отвечающей значению $u=0$. Ясно, что совмешенные лемнискаты обходятся в противоположных направлениях. Таким образом, погружение топологически эквивалентно бутылке Клейна. Если ввести параметры

$$
U=u, \quad V=\int \sqrt{2 \alpha(\widetilde{v})} d \widetilde{v}
$$

где

$$
\widetilde{v}=v+\frac{u}{2}, \quad \alpha(\widetilde{v})=\frac{a^{2}}{4} \cos ^{2} \widetilde{v}+b^{2} \cos ^{2} 2 \widetilde{v}
$$

то внутренняя метрика погружения определится линейным элементом $d s^{2}=d U^{2}+$ $d V^{2}$, т.е. является локально евклидовой.

Перейдем к описанию предложенного Д. Бланушей [22] изометрического погружения листа Мёбиуса с локально евклидовой метрикой в $E^{4}$. 
Сначала Д. Блануша строит в $E^{4}$ поверхность $S_{4}$, гомеоморфную листу Мёбиуса. Для этой цели в $E^{4}$ строится поверхность $S_{5}$, определяемая следующими параметрическими уравнениями:

$x_{1}=\rho \cos \frac{u}{2}, x_{2}=\rho \sin \frac{u}{2}, x_{3}=\frac{1}{2} \sqrt{4-\rho^{2}} \cos u, x_{4}=\frac{1}{2} \sqrt{4-\rho^{2}} \sin u, x_{5}=f(\rho)$,

где $\rho$ изменяется на интервале $(-R, R), 0<R \leqslant 2, u-$ на сегменте $[0,2 \pi]$. Функция $f(\rho)$ - четная, $f(\rho) \rightarrow \infty$ при $\rho \rightarrow R$, производная $f^{\prime}(\rho)$ непрерывна на интервале $(-R, R)$ и положительна на $(0, R)$.

Поверхность $S_{5}$ гомеоморфна листу Мёбиуса. Убедимся в этом.

Рассмотрим бесконечную полосу $\Pi=\{0 \leqslant \xi \leqslant 2 \pi,-\infty<\eta<\infty\}$. Определим отображение $S_{5}$ на полосу П с помошью соотношений: $u=\xi, \rho=\varphi(\eta)$, где $\varphi-$ функция, обратная для функции

$$
\eta=\int_{0}^{\rho} \sqrt{1+f^{\prime 2}(t)} d t
$$

Точкам $(0, \eta)$ и $(2 \pi,-\eta)$ полосы П отвечает при указанном отображении точка поверхности $S_{5}$ с координатами

$$
x_{1}=\rho, \quad x_{2}=0, \quad x_{3}=\frac{1}{2} \sqrt{4-\rho^{2}}, \quad x_{4}=\frac{1}{2} \sqrt{4-\rho^{2}}, \quad x_{5}=f(\rho) .
$$

Таким образом, $S_{5}$ гомеоморфна полосе П, у которой отождествлены точки $(0, \eta)$ и $(2 \pi,-\eta)$, т.е. $S_{5}$ гомеоморфна листу Мёбиуса.

Очевидно, что проекция $S_{4}$ поверхности $S_{5}$ на гиперплоскость $\left(x_{1}, x_{2}, x_{3}, x_{4}\right)$ также гомеоморфна листу Мёбиуса. Отсюда следует, что поверхность $S$, определяемая параметрическими уравнениями:

$$
\begin{aligned}
x_{1} & =\rho \cos \left[\frac{u}{2}+\frac{1}{2} F(\rho)-\frac{2}{R^{2}-\rho^{2}}\right], & x_{2} & =\rho \sin \left[\frac{u}{2}+\frac{1}{2} F(\rho)-\frac{2}{R^{2}-\rho^{2}}\right], \\
x_{3} & =\frac{\sqrt{4-\rho^{2}}}{2} \cos [u+F(\rho)], & x_{4} & =\frac{\sqrt{4-\rho^{2}}}{2} \sin [u+F(\rho)],
\end{aligned}
$$

где

$$
F(\rho)=\ln \left(R^{2}-\rho^{2}\right)+\frac{R^{2}}{R^{2}-\rho^{2}},
$$

гомеоморфна листу Мёбиуса (поверхность $S$ гомеоморфна $S_{4}$ ). Линейньй элемент поверхности $S$ имеет вид

$$
d s^{2}=\Phi^{2}(\rho) d \rho^{2}+[d u+\Psi(\rho) d \rho]^{2},
$$

где

$$
\Phi(\rho)=1+\frac{\rho^{2}}{4\left(4-\rho^{2}\right)}+\frac{16 \rho^{4}-4 \rho^{6}}{\left(R^{2}-\rho^{2}\right)^{4}}, \quad \Psi(\rho)=F^{\prime}-\frac{2 \rho^{3}}{\left(R^{2}-\rho^{2}\right)^{2}} .
$$

Если положить

$$
d U=\Phi(\rho) d \rho, \quad d V=d u+\Psi(\rho) d \rho
$$


то линейньй элемент будет равен $d s^{2}=d U^{2}+d V^{2}$, т.е. метрика поверхности $S$ локально евклидова. Нетрудно убедиться, что $S$ - полная поверхность [134].

В [41] А. В. Бушмелевым даны изометрические вложения полного листа Мёбиуса и бутылки Клейна в $E^{4}$ в виде поверхностей класса $C^{\infty}$. Вопрос о вложимости в классе аналитических поверхностей по-прежнему не решен.

Рассмотрим изометрическое погружение локально евклидовых метрик в евклидово пространство $E^{4}$

$$
f: U \subset E^{2} \rightarrow E^{4} .
$$

Первым нормальным пространством $N_{1}^{f}(P)$ погружения в точке $P$ называется подпространство нормального пространства $N_{P} F$, где $F=f(U)$, порожденное векторами второй квадратичной формы $F$ в точке $P$,

$$
\alpha_{f}: T_{P} F \times T_{P} F \rightarrow N_{P} F .
$$

Будем рассматривать случай, когда размерность нормального пространства постоянна, т.е. $\operatorname{dim} N_{1}^{f}=$ const.

ПрЕДЛОЖЕНИЕ 2.3.1 (Дайчер [57]). Пусть $h_{1}, h_{2}$ - вещественные функции, заданные в односвязной области $U \subset E^{2}$ и такие, что

$$
\begin{aligned}
& h_{1}\left(u_{1}, u_{2}\right)=\Phi_{1}\left(u_{1}+u_{2}\right)+\Phi_{2}\left(u_{1}-u_{2}\right), \\
& h_{2}\left(u_{1}, u_{2}\right)=-\Phi_{1}\left(u_{1}+u_{2}\right)-\Phi_{2}\left(u_{1}-u_{2}\right),
\end{aligned}
$$

где $\Phi_{1}, \Phi_{2}-$ произвольные гладкие функции одного переменного. Тогда для каждого решения $\left(v_{1}, v_{2}\right)$ в U линейной системы дифференциальных уравнений

$$
\begin{aligned}
& \frac{\partial v_{1}}{\partial v_{2}}=h_{2} v_{2}, \\
& \frac{\partial v_{2}}{\partial v_{1}}=h_{1} v_{1},
\end{aligned}
$$

такого, что везде $v_{i} \neq 0,1 \leqslant i \leqslant 2$, существует погружение $f: U \rightarrow E^{4} c$ плоской нормальной связностью и $\operatorname{dim} N_{1}^{f}=2$, а индуцированная метрика

$$
d s^{2}=v_{1}^{2} d u_{1}^{2}+v_{2}^{2} d u_{2}^{2}
$$

плоская.

Верно и обратное. Каждому изометрическому погружению плоской области

$$
f: U \subset E^{2} \rightarrow E^{4}
$$

с плоской нормальной связностью и $\operatorname{dim} N_{1}^{f}=2$ соответствуют четыре гладкие функиии $\left(v_{1}, v_{2}, h_{1}, h_{2}\right)$, где $h_{1}, h_{2}$ определяются из $(2.3 .4)$ и $\left(v_{1}, v_{2}\right)$ есть решение системь (2.3.5).

Мыговорим, что $f: U \subset E^{2} \rightarrow E^{4}$ есть композиция, если существуют изометрические погружения $g: U \subset E^{2} \rightarrow V \subset E^{3}$ и $h: V \subset E^{3} \rightarrow E^{4}$ такие, что $f=h \circ g$. Пусть $\operatorname{dim} N_{1}^{f}=1$. Тогда нормальная связность плоская. И в этом случае изометрическое погружение является композицией. 
Теорема 2.3.6 (Дайчер, Тохейро [55]). Пусть $f: U \subset E^{2} \rightarrow E^{4}$ изометрическое погружение плоской области с $\operatorname{dim} N_{1}^{f}=1$. Тогда $f$ есть композиция, $f=h \circ g$, где или $g: U \subset E^{2} \rightarrow V \subset E^{3}$, или $h: V \subset E^{3} \rightarrow E^{4}$ есть вполне геодезическое погружение.

Все плоские поверхности в $E^{4}$, которые не являются композицией, были описаны в [60]. Сначала сформулируем результат для плоских поверхностей с плоской нормальной связностью.

Пусть $\xi: U \subset E^{2} \rightarrow S^{3}(1) \subset E^{4}$ - изометрическое погружение в сферу единичного paдиуса, $\widehat{\xi}: U \rightarrow S^{3}(1)$ - полярное отображение, которое каждой точке $U$ ставит в соответствие единичную нормаль $\widehat{\xi} \in E^{4}$ поверхности $\xi(U)$; пусть $B$ - вторая квадратичная форма $\xi$ относительно нормали $\widehat{\xi}$.

Теорема 2.3.7 (До Кармо, Дайчер [60]). Пусть дано изометрическое погружение $\xi: U \subset E^{2} \rightarrow S^{3}(1) \subset E^{4}$.

Рассмотрим пару единичных векторных полей $v, \omega \in T U$ таких, что вдоль $U$ выполняются следующие требования:

1) $\omega$ - постоянное векторное поле;

2) $\langle v, B \omega\rangle=0$;

3) $\langle v, \omega\rangle \neq 0$.

Пусть $\gamma-$ произвольная гладкая функиия в $U$, которая удовлетворяет дифференциальному уравнению:

$$
\operatorname{Hess}_{\gamma}(v, \omega)+\gamma\langle v, \omega\rangle=0
$$

Положим

$$
\Omega=\frac{\operatorname{Hess}_{\gamma}(\omega, \omega)+\gamma\langle\omega, \omega\rangle}{\langle B \omega, \omega\rangle} .
$$

Определим $f: U \subset E^{2} \rightarrow E^{4}$ следующим образом:

$$
f=\gamma \xi+\xi_{*} \operatorname{grad} \gamma+\Omega \widehat{\xi}
$$

Тогда в регулярных точках $f$ есть изометрическое погружение плоской области с плоской нормальной связностью, $\operatorname{dim} N_{1}^{f}=2$ uf нигде не является композицией.

Обратно, пусть $f: U \subset E^{2} \rightarrow \subset E^{4}$ есть изометрическое погружение с плоской нормальной связностью, $\operatorname{dim} N_{1}^{f}=2 u f$ нигде не является композицией. Тогда существуют изометрическое погружение $\xi: U \subset E^{2} \rightarrow S^{3}(1) \subset E^{4}$ и единичные векторные поля $v, \omega \in T U$, удовлетворяющие условиям 1)-3), такие, что $f$ задается в виде (2.3.6), где $\gamma=\langle f, \xi\rangle$ есть опорная функция.

Для плоских погружений, которые являются композищией, справедлива

Теорема 2.3.8 (Дайчер, Тохейро [57]). Пусть $f: U \subset E^{2} \rightarrow E^{4}$ изометрическое погружение с плоской нормальной связностью $и \operatorname{dim} N_{1}^{f}=2$. Тогда следующие утверждения әквивалентны:

1) $f$ есть композиция; 
2) четыре функции $\left(v_{1}, v_{2}, h_{1}, h_{2}\right)$, ассоциированнье с $f$, равньи или

$$
\left(f_{1}\left(u_{1}\right)+\lambda f_{2}\left(u_{2}\right), f_{2}^{\prime}\left(u_{2}\right), 0, \lambda\right),
$$

uлu

$$
\left(g_{1}^{\prime}\left(u_{1}\right), \mu g_{1}\left(u_{1}\right)+g_{2}\left(u_{2}\right), \mu, 0\right),
$$

где $f_{1}\left(u_{1}\right), f_{2}\left(u_{2}\right), g_{1}\left(u_{1}\right), g_{2}\left(u_{2}\right)$ - гладкие функции одного переменного, $\lambda, \mu \in \mathbb{R}$

3) $f=h \circ g$, где g имеет вид

$$
g(s, t)=c(s)+t\left(A n(s)+v_{0}\right), \quad A \in \mathbb{R}, \quad 0 \neq v_{0} \in \mathbb{R}^{3} .
$$

Здесь $c(s)$ есть кривая, параметризованная длиной дуги с кривизной, отличной от нуля, и нормальньм вектором $n(s)$, ортогональным $v_{0}$, и $h$ есть прямой цилиндр, в котором плоскость, ортогональная $v_{0}$, есть евклидов фактор [57].

Мы говорим, что погружение $\xi: U \subset E^{2} \rightarrow S^{3}(1)$ есть поверхность типа $C$, если сушествуют два регулярных линейно независимых векторных поля на $U$ таких, что

$$
\nabla_{V}^{\prime} W=0, \quad\langle B V, W\rangle=0, \quad\langle B W, W\rangle \neq 0,
$$

где $\nabla^{\prime}$ - индуцированная связность на $\xi(U)$.

Пусть $\gamma=\langle f, \xi\rangle$ и линейное отображение $P: T U \rightarrow T U$ таково, что

$$
\langle P Z, W\rangle=\gamma\langle Z, W\rangle+\operatorname{Hess}_{\gamma}(Z, W), \quad Z, W \in T U .
$$

Теорема 2.3.9 (До Кармо, Дайчер [60]). Пусть $\xi: U \subset E^{2} \rightarrow S^{3}(1) \subset E^{4}$ eсmь поверхность типа $C$ с $\langle V, W\rangle \neq 0$. Пусть $\gamma-$ гладкая функиия, удовлетворяющая условию $\langle P V, W\rangle=0$. Положим

$$
\Omega=\frac{\langle P W, W\rangle}{\langle B W, W\rangle}
$$

Определим $f: U \rightarrow E^{4}$ как

$$
f=\gamma \xi+\xi_{*} \nabla^{\prime} \gamma+\Omega \widehat{\xi}
$$

Тогда $f$ есть плоское погружение $c \operatorname{dim} N_{1}^{f}=2$, которое нигде не является композичией, где $\xi$ есть единичное нормальное векторное поле с рангом преобразования Вейнгартена $A_{\xi}$, равным единице.

Обратно, если $f: U \subset E^{2} \rightarrow E^{4}$ - плоское погружение, которое нигде не является композичией, $u \operatorname{dim} N_{1}^{f}=2$, то существует поверхность $\xi: U \rightarrow S^{3}(1)$ типа $C$ такая, что $f$ задается (2.3.7) с $\gamma=\langle f, \xi\rangle$.

Не равенство нулю нормальной связности $R_{f}^{\perp} \neq 0$ эквивалентно условию $\nabla_{W}^{\prime} W \neq 0$.

Регулярность погружения определяется условиями:

$$
\langle P V, V\rangle \neq \Omega\langle B V, V\rangle, \quad\left\langle B\left(\nabla^{\prime} \gamma\right)+\nabla^{\prime} \Omega, W\right\rangle \neq 0 .
$$


ГипотеЗА. Пусть $F$ - компактная регулярная поверхность в $E^{4}$ с гауссовой кривизной $K=0$ и плоской нормальной связностью. Тогда это либо плоские торы в сферическом пространстве $S^{3}$, либо декартово произведение двух замкнутых кривых, которые лежат в ортогональных двумерных плоскостях.

2.4. Изометрические погружения областей плоскости Лобачевского. Изометрично непогружаема в евклидово пространство $E^{3}$ в классе регулярных поверхностей не только плоскость Лобачевского, но и полуплоскость. В классе $C^{4}$-регулярных поверхностей это доказал Н.В. Ефимов [62], а в классе регулярности $C^{2}$ это доказано в [171]. Тогда, естественно, возникает вопрос, какие области плоскости Лобачевского погружаемы в $E^{3}$.

Ф. Миндингом были найдены поверхности постоянной отрицательной кривизны, являюшиеся реализациями полос плоскости Лобачевского, заключенных между двумя эквидистантами. Э. Бельтрами построил регулярную реализацию орикруга - области, ограниченной орициклом плоскости Лобачевского. Половина псевдосферы задает регулярное изометрическое погружение орикруга.

В работе Э.Г. Позняка [135] рассмотрены изометрические погружения бесконечных многоугольников с конечным или бесконечным числом сторон, не содержащие полуплоскость. Бесконечным многоугольником назьвается пересечение замкнутых полуплоскостей, гранишы которых - прямые линии на $L^{2}$, не имеющие общих точек. Рассматривается множество многоугольников, не содержаших полуплоскость и в которых каждая сторона имеет параллельную сторону, называемую соседней. Вводятся два класса таких многоугольников $M_{1}$ и $M_{2}$. Первьй класс $M_{1}$ состоит из всех тех многоугольников, для которых сушествует орицикл $O$ в плоскости такой, что нижняя грань длин ортогональных проекций сторон на этот орищикл положительна. Второй класс $M_{2}$ состоит из всех тех бесконечных многоугольников, для которых нижняя грань длин ортогональных проекций сторон на одну из сторон положительна.

ТЕОРЕМа 2.4.1 (Позняк [135]). Любой многоугольник, принадлежащий классу $M_{1}$ или $M_{2}$, изометрически погружсается в $E^{3}$.

Доказательство этой теоремы основано на следующей геометрической идее. Поясним ее для многоугольников первого класса. Находится орицикл $O_{\alpha}$, эквидистантньй с орициклом $O$, отсекаюший все вершины многоугольника. Как известно, универсальное накрытие псевдосферы, вернее ее половины, дает изометрическое погружение некоторого орикруга, в качестве которого можно взять $O_{\alpha}$. Поэтому на части многоугольника, заключенной внутри $O_{\alpha}$, возникает регулярная чебьшёвская сеть. Остается продолжить ее на части, расположенные вне $O_{\alpha}$. Соединим вершины многоугольника с бесконечно удаленной точкой $Q$ орикруга $O_{\alpha}$ геодезическими линиями $a_{i}$ и построим бесконечную эквидистантную полосу вдоль $a_{i}$ ширины $2 h$. При некоторой ширине $2 h$ часть многоугольника, оставшаяся вне орикруга $O_{\alpha}$, попадет в эту полосу. В этой полосе вводится полугеодезическая система координат, и в ней записьвается система уравнений погружения. Начальные данные для решения этой системы задаются на $a_{i}$ так, что на части $a_{i}$, лежашей вне $O_{\alpha}$, они продолжают значения искомых функций на части $a_{i}$ внутри $O_{\alpha}$, которые соответствуют изометрическому погружению псевдосферы.

Далее используется теорема сушествования и единственности решения системы по- 
гружения в полосе, доказанная Э.Г. Позняком. Таким образом, можно получить погружения многоугольников класса $C^{\infty}[10]$.

Допускают регулярную реализацию следуюшие некомпактные области плоскости Лобачевского [100], [101]:

а) расширяющаяся полоса с геодезической базой;

б) вьпуклая область, содержащая два орикруга;

в) вьпуклая область, граница которой имеет любой (конечный) порядок соприкосновения с абсолютом;

г) новьй класс бесконечных вьпуклых многоугольников.

Пусть

$$
\varphi(y)=\lambda \sqrt{y^{2}+a^{2}}-\left(2 \lambda+\nu+\frac{1}{6}\right)
$$

есть функция, определяющая расширяюшуюся полосу

$$
a=\text { const }>11, \quad \lambda=\text { const }>\frac{3}{a-2},
$$

$y$ - длина на геодезической, которая есть база полосы, $\nu=\ln \left(29 \lambda^{2}+24 \lambda+3\right)$.

Теорема 2.4.2 (Кайдасов, Шикин [100], [101]). Расширяющаяся полоса $\pi(\varphi)$ с функцией $\varphi(y)$ реализуется в $E^{3}$ в виде регулярной $\left(C^{3}\right.$-регулярной $)$ поверхности.

Теорема 2.4.3 (Кайдасов, Шикин [100], [101]). Любъе два орикруга можно заключить в погружаемую в $E^{3}$ (регулярно и изометрично) выпуклую область.

Теорема 2.4.4 (Кайдасов, Шикин [100], [101]). На плоскости Лобачевского существует неограниченная выпуклая область $Q$, обладающая следующими свойствами:

1) область $Q$ содержит некоторьй орикруг;

2) никакой орикруг не вмещает всех точек области $Q$;

3) область $Q$ не содержит полной геодезической;

4) область $Q$ реализуется в $E^{3}$ в виде регулярной $\left(C^{3}\right.$-гладкой) поверхносmu.

Доказана возможность изометрического погружения в $E^{3}$ нового класса бесконечных вьпуклых многоугольников. Эти многоугольники характеризуются тем, что их стороны касаются гранищы расширяюшейся полосы, базой которой является одна из сторон многоугольника.

Для доказательства возможности погружения в $E^{3}$ многоугольников указанного вида решение основной системы строится в специально приспособленной расширяющейся полосе, а затем продолжается на содержашую многоугольник область методом, разработанньм Э.Г. Позняком.

Д. Блануша получил конкретные параметрические уравнения поверхности класса $C^{\infty}$ без самопересечений в $E^{6}$, внутренняя метрика которой совпадает с метрикой плоскости Лобачевского. Пусть

$$
\psi_{1}(u)=e^{2[(|u|+1) / 2]+5}, \quad \psi_{2}(u)=e^{2[|u| / 2]+6},
$$


где [· ] означают целую часть заключенного в них выражения. Положим

$$
\begin{gathered}
A=\int_{0}^{1} \sin \pi \xi e^{-1 / \sin ^{2} \pi \xi} d \xi \\
\varphi_{1}(u)=\left(\frac{1}{A} \int_{0}^{u+1} \sin \pi \xi e^{-1 / \sin ^{2} \pi \xi} d \xi\right)^{1 / 2} \\
\varphi_{2}(u)=\left(\frac{1}{A} \int_{0}^{u} \sin \pi \xi e^{-1 / \sin ^{2} \pi \xi} d \xi\right)^{1 / 2} \\
f_{1}(u)=\frac{\varphi_{1}(u)}{\psi_{1}(u)} \operatorname{sh} u, \quad f_{2}(u)=\frac{\varphi_{2}(u)}{\psi_{2}(u)} \operatorname{sh} u
\end{gathered}
$$

Пусть $x_{i}(i=1, \ldots, 6)$ - декартовы координаты в $E^{6}$. Тогда вложение в $E^{6}$ плоскости Лобачевского с линейньм элементом

$$
d s^{2}=d u^{2}+\operatorname{sh}^{2} u d v^{2}
$$

задается следующими параметрическими уравнениями:

$$
\begin{aligned}
x_{1} & =\int_{0}^{u} \sqrt{1-f_{1}^{\prime 2}(\xi)-f_{2}^{\prime 2}(\xi)} d \xi, \\
x_{2} & =v \\
x_{3} & =f_{1}(u) \cos \left(v \psi_{1}(u)\right), \\
x_{4} & =f_{1}(u) \sin \left(v \psi_{1}(u)\right), \\
x_{5} & =f_{2}(u) \cos \left(v \psi_{2}(u)\right), \\
x_{6} & =f_{2}(u) \sin \left(v \psi_{2}(u)\right) .
\end{aligned}
$$

То, что линейньй элемент совпадает с линейным элементом плоскости Лобачевского, устанавливается путем тонких аналитических рассуждений [23].

ТеОрема 2.4.5 (Розендорн [139]). Плоскость Лобачевского допускает регулярное изометрическое погружение в $E^{5}$.

Теорема 2.4.6 (Сабитов [145]). Плоскость Лобачевского можно изометрично погрузить в $E^{4}$ в виде кусочно аналитической поверхности гладкости в иелом класса $C^{0,1}$. Но в то же время плоскость Лобачевского не допускает изометрического погружения в $E^{4}$ в виде обобщенной поверхности вращения.

В [140] Э.Р. Розендорном доказано, что плоскость Лобачевского не допускает изометрического погружения в $E^{4}$ в виде регулярной геликоидальной поверхности. В [114] этот результат обобщен на геликоидальные погружения $L^{2}$ в конечномерное евклидово пространство $E^{n}$, в [112] - на геликоидальные погружения $L^{l}$ в $E^{n}$.

В [23], [24], [26] Д. Бланушей даны явные $C^{\infty}$-изометрические вложения:

1) плоскости Лобачевского в сфферическое пространство $S^{8}$;

2) цилиндра с гиперболической метрикой постоянной гауссовой кривизны -1 в евклидово пространство $E^{7}$, в сферическое пространство $S^{8}$;

3) бесконечного листа Мёбиуса с гиперболической метрикой в евклидово пространство $E^{8}$, в сферическое пространство $S^{10}$ (см. также [138]). 
В [73], используя комплексную структуру, индуцированную второй квадратичной формой, дано конформное представление внутренне плоских поверхностей (т.е. с гауссовой кривизной $K=0$ ) в трехмерном гиперболическом пространстве. Задача эквивалентна нахождению решений уравнения Монжа-Ампера

$$
z_{x x} z_{y y}-z_{x y}^{2}=1
$$

\section{5. Изометрические погружения с постоянной внешней геометрией.}

Интересен вопрос о связи групы движений пространства Лобачевского и групшы движений евклидова пространства. Естественной формулировкой этого вопроса является вопрос о возможности таких изометрических погружений пространств Лобачевского в евклидово пространство, при которых какая-либо группа движений пространства Лобачевского может быть индуцирована движениями объемлюшего евклидова пространства. По-видимому, впервые на этот вопрос обратил внимание Л. Бибербах [17]. Им было построено такое изометрическое погружение плоскости Лобачевского в гильбертово пространство, при котором любое внутреннее движение плоскости Лобачевского реализуется с помощью соответствуюшего движения гильбертова пространства (примером такого типа погружений в $E^{3}$ метрик нулевой и постоянной положительной кривизны могут служить плоскость и сфрера).

Л. Бибербах построил аналитическое вложение $L^{2}$ в гильбертово пространство. Это вложение задается явньми формулами. Прежде, чем их вьписать, введем вспомогательное обозначение. Пусть $F_{m}(z)$ - конечньй или бесконечный набор функций комплексного аргумента $z=u+i v$, возможно не аналитических. Положим

$$
x_{2 m-1}=\operatorname{Re} F_{m}(z), \quad x_{2 m}=\operatorname{Im} F_{m}(z), \quad m=1, \ldots, p
$$

считая, что $x_{j}$ - декартовы координаты в $E^{N}, N \geqslant 2 p$.

Конструкция Бибербаха выглядит очень просто: в (2.5.1) берем $F_{m}(z)=m^{-1 / 2} z^{m}$, $p=N=\infty$. Подсчет показывает, что в результате при $p=|z|<1$ получается вложение в $E^{\infty}$ метрики $d s^{2}=\left(1-p^{2}\right)^{-2}\left(d u^{2}+d v^{2}\right)$, а это, как известно, - одна из форм записи метрики $L^{2}$. При этом условия $x_{1}=u, x_{2}=v$ соблюдены, а поверхность однолистно проектируется на круг $p<1$ плоскости $x_{1}, x_{2}$.

Л. Бибербах доказал, что построенная им поверхность вида (2.5.1) не лежит ни в каком подпространстве конечной размерности и обладает следуюшим интересным свойством: вся группа движений индущируется на ней движениями объемлющего пространства. В той же работе он изложил принадлежашее Шмидту доказательство того, что в $E^{N}(N<\infty)$ на погруженной $L^{2}$ группа всех ее движений не может быть индуцирована движениями $E^{N}$, хотя тогда еще не было известно, возможны ли такие погружения.

В [18] дано изометрическое вложение $n$-мерного пространства Лобачевского в гильбертово пространство. Однако там не рассматривался вопрос: индуцируются ли все движения данного вложения пространства Лобачевского движениями объемлющего гильбертова пространства. Как мне кажется, этот вопрос остается открытьм.

Дальнейшему изучению этих вопросов посвящена работа С. Б. Кадомцева [97]. 
ОПРЕДЕЛЕНИЕ. Поверхность $\Omega \in E^{n}$ назьвается поверхностью вращения $с$ полюсом в точке $M \in \Omega$, если для любых двух геодезических $l_{1}, l_{2}$, выходяших из точки $M$, сушествует такое движение $G$ пространства $E^{n}$, что

1) $G(M)=M$

2) $G(\Omega)=\Omega$

3) $G\left(l_{1}\right)=l_{2}$.

Никакая часть плоскости Лобачевского не может быть погружена в $E^{3}$ в виде поверхности врашения с полюсом. Однако такое погружение оказьвается возможным уже в $E^{4}$.

Теорема 2.5.1 (Кадомцев [97]). Плоскость Лобачевского L ${ }^{2}$ не может бить погружена в иелом ни в какое конечномерное евклидово пространство $E^{n}{ }_{\boldsymbol{\theta}}$ виде поверхности вращения с полюсом.

Полное пространство Лобачевского $L^{m}(m \geqslant 2)$ не допускает такого погружения в конечномерное евклидово пространство $E^{n}$, при котором любое внутреннее вращение $L^{m}$ вокруг фиксированной точки $M \in L^{m}$ может быть индуцировано каким-либо движением объемлющего пространства $E^{m}$.

Из теоремы 2.5.1, в частности, следует невозможность такого погружения плоскости Лобачевского $L^{2}$ в конечномерное евклидово пространство, при котором любое внутреннее движение $L^{2}$ может быть индуцировано каким-либо движением объемлющего пространства.

ОПРЕДЕЛЕНИЕ. Поверхность $\Omega \in E^{n}$ постоянной кривизны называется поверxностью с параллельным переносом, если для любых двух точек $P$ и $Q$ поверхности $\Omega$ сушествует такое движение $G$ пространства $E^{n}$, что 1) $\left.G(\Omega)=\Omega, 2\right) G(L)=L$, $G(l)=l, 3) G(P)=Q$, где $l$ - геодезическая линия поверхности $\Omega$, проходяшая через точки $Р$ и $Q$.

Теорема 2.5.2 (Кадомцев [97], [99]). Плоскость Лобачевского L ${ }^{2}$ не может быть погружена ни в какое конечномерное евклидово пространство в виде поверхности с параллельным переносом.

Теорема 2.5.3 (Кадомцев [98]). Полная плоскость Лобачевского не может быть погружена в классе $C^{0}$ ни в какое конечномерное евклидово пространство так, что какая-либо непрерывная нетривиальная ее подгруппа движений индущируется движсениями евклидова пространства.

Заметим, что центральное место в доказательстве этих предложений занимает доказательство некоторого вспомогательного утверждения, представляющего, однако, и самостоятельный интерес. Суть его в следуюшем. Пусть $L-$ кривая класса $C^{0}$ в евклидовом пространстве, обладаюшая свойством: для любых двух точек кривой $L$ существуют окрестности этих точек, конгруэнтные друг другу (примерами таких кривых в $E^{3}$ могут служить прямая, окружность и винтовая линия). Тогда кривая $L$ аналитическая. 
Поясним кратко идею доказательства теоремы 2.5.3. Предполагая противное, допустим, что указанное погружение возможно. Тогда метрику соответствующей поверхности можно привести к виду

$$
d l^{2}=d s^{2}+H^{2}(s) d t^{2},
$$

где координатные линии $t$ являются одновременно траекториями некоторой однопараметрической групш движений плоскости Лобачевского и некоторой однопараметрической группы движений объемлюшего евклидова пространства. Учитьвая вид движений объемлющего пространства, можно получить для функции $H(s)$ следующую оценку: $|H(s)| \leqslant C s^{2}$ при достаточно больших $s$. С другой стороны, функция $H(s)$, очевидно, экспоненциально возрастает. Полученное противоречие доказьвает утверждение (см. также [10]).

Естественно изучить те поверхности, в которых все внутренние движения индуцируются движениями объемлюшего евклидова пространства.

Двумерная поверхность $F^{2} \subset E^{n}$ называется поверхностью с постоянной внешней кривизной, если для любых двух точек $X \in F^{2}, Y \in F^{2}$ существуют окрестности $U(X)$ и $U(Y)$ на поверхности, конгруэнтные в $E^{n}$.

ТЕорема 2.5.4 (Талеб [74]). Единственные двумерные поверхности с постоянной внешней геометрией в $E^{4}$ класса регулярности $C^{3}$ суть плоскости, сферы, обобщенные торы Клиффорда и чилиндрические поверхности, направляющие которых - винтовые линии в некотором $E^{3}$, а прямолинейнье образующие - ортогональные $к E^{3}$.

ТЕОРема 2.5.5 (Талеб [75]). Если двумерная поверхность $E^{5}$ с положительной гауссовой кривизной имеет постоянную внешнюю геометрию, то она будет сферой или поверхностью Веронезе.

ТЕОРема 2.5.6 (Талеб [75]). Все двумерные поверхности с постоянной внешней геометрией и нулевой гауссовой кривизной в $E^{5}$ представляются в виде:

$$
\begin{aligned}
x_{j}=\sum_{i=1}^{2} & {\left[\cos \gamma_{i} v \sum_{k=1}^{2}\left(a_{j, 2 k-1}^{i} \cos \delta_{k} u+a_{j, 2 k}^{i} \sin \delta_{k} u\right)\right.} \\
& \left.\quad+\sin \gamma_{i} v \sum_{k=1}^{2}\left(a_{j, 2 k+3}^{i} \cos \delta_{k} u+a_{j, 2 k+4}^{i} \sin \delta_{k} u\right)\right]
\end{aligned}
$$

$j=1,2,3,4, x_{5}=C_{1} u+C_{2} v$, де $a_{j k}^{i}, C_{1}$ и $C_{2}$ определяются через параметры эллипса нормальной кривизнь и коэффичиенты кручения поверхности.

Естественно найти все двумерные и многомерные поверхности постоянной секционной кривизны, у которых все внутренние движения индуцируются движениями объемлющего пространства. Это связано с минимальными погружениями многомерных сфер $S_{K(s)}^{n}$ в $S_{1}^{m(s)}$, где $K(s)=n / s(s+n-1)$ и

$$
m(s)=(2 s+n-1) \frac{(s+n-2) !}{s !(n-1) !}-1 .
$$


Эти погружения строятся с помошњю сферических гармоник порядка $s$, т.е. ограничения на $S_{1}^{n}$ однородных полиномов $P\left(x_{0}, x_{1}, \ldots, x_{n}\right)$ степени $s$ в $E^{n+1}$, удовлетворяющих уравнению

$$
\sum_{i} \frac{\partial^{2} P}{\partial x_{i}^{2}}=0 .
$$

Размерность пространства сферических гармоник порядка $s$ равна $m(s)+1$, и если $f_{0}, \ldots, f_{m}$ - ортонормированньй базис этого пространства, то искомое изометрическое погружение $M \rightarrow E^{m+1}$ имеет вид

$$
\psi(p)=\left(f_{0}(p), \ldots, f_{m}(p)\right) .
$$

2.6. Погружение проективной плоскости. Из теоремы 2.2 .5 следует, что изометрическое регулярное погружение стандартной сферы $S^{2}(1)$ в $S^{3}(1)$ есть только вполне геодезическая сфера. С другой стороны, Клиффордов плоский тор $T^{3}$ изометрически вкладьвается в сферу $S^{5} \subset E^{6}$, а значит, погруженная сфера $S^{2}(R) \subset T^{3}$ радиуса $R$ изометрически погружается в $S^{5}(1)$. В [72] изометрично не стандартно погрузили сферы $f: S^{2}(R) \rightarrow S^{4}, 0<R<4$, причем при $R<1.6$ погружение является вложением.

Вешественная проективная плоскость $\mathbb{R} P^{2}$ допускает метрику постоянной положительной кривизны. Покажем, что она не допускает изометричного вложения класса $C^{2}$ в евклидово пространство $E^{4}$.

Как показал Уитни, вешественная проективная плоскость не допускает дифференциального вложения в $E^{4}$ с нормальньм векторньм полем без особенностей. Поэтому нам достаточно доказать, что при изометрическом $C^{2}$-погружении двумерного риманова многообразия положительной кривизны в $E^{4}$ всегда сушествует нормальное векторное поле без особенностей.

Пусть $f: V \rightarrow E^{4}$ - такое погружение. Обозначим через $T_{v}$ касательную плоскость к $V$ в точке $v \in V$, а через $N_{v}$ - нормальную плоскость и построим для произвольного вектора $\xi \in N_{v}$ “вторую квадратичную форму" ( $\left.d_{v}^{2} f, \xi\right)$. Это квадратичная форма на $T_{v}$, обладающая следом $\sigma(\xi)$ и дискриминантом $\delta(\xi)$. След $\sigma$ представляет собой линейную форму на $N_{v}$, а дискриминант $\delta$ - квадратичную форму на $N_{v}$. Последняя обладает своим собственным следом, который согласно формуле Гаусса равен кривизне многообразия $V$ в точке $v$ и поэтому положителен. Так как квадратичная форма с положительным следом принимает положительные значения, то $\delta(\xi)>0$ при некотором $\xi \in N_{v}$, а так как бинарная квадратичная форма с положительньм дискриминантом имеет ненулевой след, то $\sigma(\xi) \neq 0$ при этом $\xi$. Таким образом, $\sigma-$ ненулевая линейная форма на $N_{v}$. Эта форма непрерьвно зависит от $v$, и потому ей отвечает дуальное векторное поле, которое и будет требуемым нормальным векторным полем без особенностей [80].

Изометрическое вложение $\mathbb{R} P^{2}$ кривизны $\frac{1}{r^{2}}$ в $S^{4}(\rho) \subset E^{5}, \rho=\frac{r}{\sqrt{3}}$, задается следуюшим радиус-вектором [21]:

$$
\begin{gathered}
x_{1}=\frac{r}{2} \sin ^{2} u \sin 2 v, \quad x_{2}=\frac{r}{2} \sin ^{2} u \cos 2 v, \\
x_{3}=\frac{r}{2} \sin ^{2} u \sin v, \quad x_{4}=\frac{r}{2} \sin ^{2} u \cos v, \quad x_{5}=\frac{\rho}{4 \sqrt{3}}(1+3 \cos 2 u) .
\end{gathered}
$$


Это есть поверхность Веронезе. В [21] показано, что вещественное проективное пространство постоянной гауссовой кривизны $k=\frac{1}{r^{2}}$ можно изометрически погрузить в сферу $S^{4}(\rho), \rho>r / 3$, в вещественное проективное пространство $\mathbb{R} P^{4}(\rho)$ постоянной кривизны $K=\frac{1}{\rho^{2}}$, т.е.

$$
k \geqslant \frac{K}{3}
$$

Возможно ли погружение при $k<\frac{K}{3} ?$

Дается также погружение $\mathbb{R} P^{2}$ в евклидово пространство $E^{4}$ и пространство Лобачевского $L^{4}$.

\section{Глава 3. Изометрические погружения многомерных пространственных форм}

3.1. Изометрические погружения пространственных форм подмногообразиями нулевой внешней кривизны. Пусть $F^{l}$ есть регулярное класса $C^{2}$ подмногообразие в евклидовом пространстве $E^{l+p}$ с нулевой секционной кривизной. Тогда $F^{l}$ локально изометрично евклидову пространству.

Пусть $F^{l}$ - регулярное класса $C^{2}$ подмногообразие в римановом пространстве $M^{n}$, $Q \in F^{l}$ - точка на подмногообразии, $\xi$ - единичное нормальное векторное поле в окрестности точки $Q, X, Y$ - векторные поля, касательные к многообразию, $\bar{\nabla}$ - связность Леви-Чивита в римановом многообразии $M^{n}, \nabla$ - индуцированная связность на $F^{l}, \nabla^{\perp}$ - связность в нормальном расслоении $F^{l}, \alpha(X, Y)$ - векторная вторая квадратичная форма $F^{l}, A_{\xi}$ - линейное преобразование в $T_{Q} F^{l}$, соответствующее второй квадратичной форме относительно нормали $\xi$.

ОПРЕДЕЛЕНИЕ. Внешний нуль-индекс $\mu(Q)$ точки $Q \in F^{l}$ есть максимальная размерность подпространства $L(Q)$ касательного пространства $T_{Q} F^{l}$ такого, что

$$
A_{\xi} y=0
$$

для любого вектора $y \in L(Q)$ и для любой нормали $\xi \in N_{Q} F^{l}$ в этой точке.

Подмногообразие назьвается $k$-сильно параболическим, если внешний нуль-индекс $\mu \geqslant k$ для каждой точки подмногообразия.

С. Черн и Н. Кейпер [48] доказали, что для подмногообразия $F^{l}$ нулевой внешней кривизны в римановом пространстве $M^{l+p}$ внешний нуль-индекс удовлетворяет неравенству

$$
\mu \geqslant l-p
$$

при коразмерности $p<l, \mu>0$.

Если в окрестности точки $Q$ нуль-индекс $\mu$ постоянен, то на поверхности класса регулярности $C^{3}$ мы имеем дифференцируемое нуль-распределение $L(Q)$. Однако без дополнительных требований для поверхностей в римановом пространстве это распределение может быть даже неинтегрируемьм.

Пусть вдоль подмногообразия $F^{l}$ тензор кривизны объемлющего риманова многообразия $M^{n}$ удовлетворяет условию:

$$
R(X, Y) Z^{\perp}=0
$$


где $R$ - оператор кривизны, $X, Y, Z$ - векторы, касательные к $F^{l}, \perp$ - ортогональная проекция на нормальное пространство к $F^{l}$.

Для пространства постоянной кривизны $C$

$$
R(X, Y) Z=C(\langle X, Z\rangle Y-\langle Y, Z\rangle X)
$$

и условие (3.1.2) удовлетворяется вдоль произвольного подмногообразия.

Лемма 3.1.1 (Мальтц [113]). Пусть $F^{l}$ есть регулярное класса $C^{3}$ подмногообразие в римановом пространстве $M^{n}$. Предположим, что в окрестности точки $P_{0} \in F^{l}$ нуль-индекс $\mu(Q)=k=$ const $u$ в точках подмногообразия вьполняется условие (3.1.2). Тогда нуль-распределение $L(Q)$ интегрируемо и слои $S L(Q)$ являются вполне геодезическими подмногообразиями обгемлющего пространства $M^{n}$.

Нормальное пространство стационарно вдоль слоев, т.е. произвольная нормаль $\xi(Q)$ в точке $Q$ слоя $S L(Q)$ после параллельного перенесения в связности обгемлющего пространства вдоль пути $\gamma \in S L(Q)$ остается нормалью к подмногообразию $F^{l}$.

Если $F^{l}$ есть полное подмногообразие и $\mu\left(P_{0}\right)=\min _{Q \in F^{l}} \mu(Q)=k$, то слой $S L\left(P_{0}\right)$ является полным вполне геодезическим подмногообразием.

Если обгемлющее пространство есть пространство постоянной кривизны, то слои являются вполне геодезическими подмногообразиями постоянной кривизны: в случае евклидова пространства - большими сферами, в случае пространства Лобачевского - пространствами Лобачевского меньшей размерносmu.

Если на локально евклидовом подмногообразии $F^{l}$ возьмем точку $Q$, в окрестности которой внешний нуль-индекс постоянен, тогда из леммы 3.1.1 следует, что через точку $Q$ проходит $\mu$-мерная плоскость объемлющего евклидова пространства, вдоль которой нормальное пространство стационарно.

Если подмногообразие $F^{l}$ полно и точка $Q \in F^{l}$ такая, что

$$
\mu_{0}=\mu_{0}(Q)=\min _{Q \in F^{l}} \mu(Q)
$$

то из леммы 3.1 .1 следует, что на полной поверхности $F^{l}$ лежит евклидова плоскость $E^{\mu_{0}}$.

Имеет место

Лемма 3.1 .2 ([34]). Пусть подмногообразие $F^{l}$ является изометрическим погружением класса $C^{0}$ полного риманова многообразия $M^{l}$, которое является метрическим произведением $M^{l}=M^{l-k} \times E^{k}$, где $E^{k}-$ евклидов фактор.

Если образ $\left(Q_{0} \times E^{k}\right)$, где $Q_{0}$ - фиксированная точка $M^{l-k}$, содержит $k$ линейно независимых прямых евклидова пространства, то подмногообразие $F^{l}$ является иилиндром с $k$-мерной образующей.

Эта лемма следует из леммы 2.1.1. Учитьвая оценку (3.1.1), для локально евклидовых метрик из лемм 3.1.1, 3.1.2 следует 
Теорема 3.1 .1 (Хартман [81]). Пусть $F^{l}$ есть полное регулярное локально евклидово подмногообразие в евклидовом пространстве $E^{l+p}, p<l$. Тогда $F^{l}$ есть иилиндр с $(l-p)$-мерной образующей.

При $p=1$ это теорема Хартмана-Ниренберга [58]. При $p=l$ тор Клиффорда несет локально евклидову метрику и является компактньм подмногообразием. Из теоремы 3.1.1 следует теорема Томпкинса, которая утверждает, что при $p<l$ не сушествует компактного подмногообразия $F^{l}$ с нулевой секционной кривизной в $E^{l+p}[48]$.

Мне кажется, интересно оценить размерность евклидова пространства $E^{l+p}$, в которое изометрично погружается плоская пространственная форма $M^{l}$, в зависимости от сложности фундаментальной групшы $\pi_{1}\left(M^{l}\right)$.

Пусть $F^{l}$ есть изометрическое погружение стандартной сферы кривизны единища $S^{l}(1)$ в сфферу $S^{l+p}(1)$. Подмногообразие $F^{l}$ также будет сильно параболическим подмногообразием в сферическом пространстве $S^{l+p}$, и если $p<l$, то с той же самой оценкой на внешний нуль-индекс и с аналогичньп строением. Только плоскости евклидова пространства заменяются вполне геодезическими сфферами. И если поверхность $F^{l}$ полная, то на ней лежит вполне геодезическая сфера $S^{l-p}$. Имеет место

Лемма 3.1 .3 ([27], [30]). Пусть $F^{l}$ - гладкая класса $C^{1}$ поверхность, изометричная $S^{l}$ в сферическом пространстве $S^{n}$. Eсли на $F^{l}$ лежст большая окружность, то поверхность $F^{l}$ является вполне геодезической большой сферой.

Для случая $l=2$ это есть теорема 2.2.5.

Теперь рассмотрим общий случай. Пусть $S^{1}-$ большая окружность $S^{l+p}$, которая лежит на $F ; F_{l-2}-$ множество поверхности $F$, полярное $S^{1}$ на $F$. Оно лежит в сфере $S^{l+p-2}$, полярной $S^{1}$ на $S^{l+p}$.

Пусть $X, Y$ - произвольные фиксированные диаметрально противоположные точки $S^{1}, Z \in F_{l-2}$. Полуокружности с концами $X, Y$ на $S^{l+p}$, проходящие через $Z \in$ $F_{l-2}$, лежат на $F$ и образуют поверхность $F_{l-1}$, изометричную $S^{l-1}$. Покажем, что $F_{l-1}$ - гладкая поверхность в точке $X$.

Поверхность $F$ получается из поверхности $F_{l-1}$ вращением. При этом врашении точки $F_{l-2}$ остаются неподвижными, а точка $X$ движется по $S^{1}$. Из предположения, что $F_{l-1}$ негладкая, следует, что $F$ также негладкая, а это противоречит условию леммы.

Итак, $F_{l-1}$ - гладкая в точке $X$ поверхность - будет просто большой сферой $S^{l-1}$, а значит, $F$ будет большой сферой $S^{l}$ в $S^{l+p}$.

И аналог теоремы 3.1 .1 для сфферического пространства есть

ТеОрема 3.1 .2 ([27], [30]). Пусть $F^{l}$ есть регулярное класса $C^{2}$ изометрическое погружение стандартной сферь кривизнь 1 в сферическое пространство $S^{l+p}(1)$. Если $p<l$, то $F^{l}$ есть вполне геодезическая больиая сфера.

Теорема 3.1.2 была передоказана Д. Ферусом в [70].

Соответствующим аналогом для гиперболических пространств является

Теорема 3.1 .3 (Зегиб [177]). Пусть $f: V^{l}(-1) \rightarrow W^{l+p}(-1)$ есть регулярное изометрическое погружение компактного гиперболического пространства кривизны -1 в гиперболическую пространственную форму той жсе кривизны. Если $p<l$, то $f(V)$ есть вполне геодезическое подмногообразие. 
Так как внешний нуль-индекс $\mu \geqslant l-p>0$, то в окрестности точки с минимальным нуль-индексом $\mu_{0}$ мы получаем на $f(V)$ геодезическое слоение с полными слоями (лемма 3.1.1). А. Зегибом также доказано, что на гиперболическом многообразии конечного объема (не обязательно полном) не существует $C^{1}$-геодезического слоения с полными слоями. Отсюда следует, что $\mu_{0}=l$ и $f(V)$ является вполне геодезическим подмногообразием [178].

Изометрические погружения пространства Лобачевского $L^{l}(-1)$ в $L^{l+1}(-1)$ изучались Д. Ферусом в [69]. Эти подмногообразия являются сильно параболическими. Если внешний нуль-индекс $\mu(Q)=l-1$ для всех точек, то кривая, ортогональная к полным вполне геодезическим слоям нуль-распределения, имеет кривизну не больше 1, где кривизна взята в метрике поверхности.

Более того, доказана

ТЕОРема 3.1 .4 (Ферус [69]). Каждому вполне геодезическому гиперслоению пространства Лобачевского $L^{l}$ отвечает изометрическое погружение $L^{l}$ в $L^{l+1}$, при котором слои переходят в слои нуль-распределения $(l-1)$-сильно параболической гиперповерхности, и погружение не имеет омбилических точек, т.е. внешний нуль-индекс во всех точках равен $\mu(Q)=l-1$.

С. Александер и Е. Портной [6] уточнили внешнее строение изометрического погружения $H^{l} \rightarrow H^{l+1}$ с внешним нуль-индексом $\mu(Q)=l-1$ во всех точках. Пусть $\bar{L}^{l+1}=L^{l+1} \cup L_{\infty}^{l}$, где $L_{\infty}^{l}-$ идеальная граница.

Теорема 3.1 .5 (Александер, Портной [6]). Пусть $\eta: L^{l} \rightarrow L^{l+1}$ есть свободное от омбилических точек изометрическое погружение $u\left\{L_{c}\right\}_{c \in I}$ - семейство образуюших $\eta$. Тогда существует единственная кривая $\sigma: I \rightarrow L^{n+1}$ такая, что образующие $\left\{L_{c}\right\}$ параллельны в $\bar{L}^{n+1}$ вдоль кривой $\sigma$.

Эта формулировка, в некотором смысле, аналогична теореме Хартмана-Ниренберга, утверждающей, что изометрическое погружение евклидова пространства $E^{l}$ в $E^{l+1}$ есть цилиндр с $(l-1)$-мерной образуюшей.

Цилиндрические погружения $L^{l}$ в $L^{l+p}$ как частный случай сильно параболических подмногообразий, изучались в [36], [35]. Верна

ТеОРема 3.1 .6 ([35]). Пусть $F^{l}$ - изометрическое погружение пространства Лобачевского $L^{l}(-1)$ в пространство Лобачевского $L^{l+p}(-1)$ с постоянным внешним нуль-индексом $\mu$. Пусть $p<l$ и существует поверхность, ортогональная нуль-слоям.

Если поверхность, ортогональная нуль-слоям, является вполне омбиличной как подмногообразие $F^{l}$ (если она вырождается в кривую, то требуется постоянство геодезической кривизны), то $F^{l}$ есть иилиндрическая поверхность в пространстве Лобачевского $L^{l+p}(-1)$, которая в интерпретачии Кәли-Клейна изображсатся иилиндром $c(k-1)$-мерной образующей $(k \geqslant l-p)$ над конической поверхностью с вершиной либо на абсолюте, либо вне его.

Теорема верна без ограничения на коразмерность $p$, если изометрическое погружение осуществляется в классе сильно $k$-параболических подмногообразий с постоянным нуль-индексом. 
В [2] изучались изометрические погружения $L^{l}$ в $L^{l+1}$ и с омбилическими точками (в этом случае с точками уплощения), т.е. внешний нуль-индекс $\mu(Q)$ может равняться и $l$. Для формулировки теоремы дадим определение ламинации.

ОПРЕДЕЛЕНИЕ. Пусть $U \subset L^{n}$ есть не более чем счетное объединение открытых связных компонент, каждая из которых $C^{\infty}$-расслоена на полные вполне геодезические гиперповерхности. Мы обозначим это слоение $F_{U}, L^{n}-U$ есть замкнутое множество точек. Тройка $\left(U, F_{U}, L^{n}-U\right)$ есть $C^{\infty}$-ламинация на $L$.

С каждьм изометрическим погружением $f: L^{l} \rightarrow L^{l+1}$ ассоциирована ламинация, где $U$ есть множество точек с внешним нуль-индексом $\mu(Q)=l-1, F_{U}$ - слоение нуль-распределений второй квадратичной формы, $L^{n}-U$ - точки уплощения с $\mu(Q)=l$.

Верно и обратное.

ТЕОРема 3.1 .7 (Эйб, Хаас [2]). Для каждой дифферениируемой ламиначии на $L^{n}$ существует семейство изометрических погружсений $L^{n}$ в $L^{n+1}$ такое, что индуцированное погружение нуль-слоения на вполне геодезические гиперповерхности множества $U$ полностью совпадает со слоением $F_{U}$ ламиначии, а точки уплощения - смножеством $L^{n}-U$.

Полная параметризация изометрических погружений $L^{l} \rightarrow L^{l+1}$ дана в [5], где пространство изометрических погружений $L^{l} \rightarrow L^{l+1}$ параметризуется семейством из не более чем счетного числа соответственно выбранных $n$-строчек вещественнозначных функций, определенных на открытом интервале. В [86] реализован аналитический подход к этой проблеме, как это было сделано ранее для двумерного случая [88]. В этом случае каждому изометрическому погружению ставится в соответствие решение вырожденной системы уравнений типа Монжа-Ампера в единичном шаре $B^{n}(1)$.

ТЕОРема 3.1 .8 (Ху [90]). Каждому гладкому изометрическому погружению $L^{n}(-1) \rightarrow L^{n+1}(-1)$ соответствует решение системы уравнений

$$
\begin{gathered}
\frac{\partial^{2} u}{\partial \xi_{i} \partial \xi_{j}} \cdot \frac{\partial^{2} u}{\partial \xi_{k} \partial \xi_{l}}-\frac{\partial^{2} u}{\partial \xi_{i} \partial \xi_{k}} \cdot \frac{\partial^{2} u}{\partial \xi_{j} \partial \xi_{l}}=0, \\
\xi=\left(\xi_{1}, \ldots, \xi_{n}\right) \in B^{n}(1), \quad i, j, k, l=1, \ldots, n .
\end{gathered}
$$

Обратно, для каждого гладкого решения U системы (3.1.3) мы определим

$$
\begin{cases}g_{i j}=\lambda^{-4}\left(\lambda^{2} \delta_{i j}+\xi_{i} \xi_{j}\right), & \lambda=\sqrt{1-\xi_{1}^{2}-\cdots-\xi_{n}^{2}} \\ h_{i j}=\lambda^{-1} \frac{\partial^{2} u}{\partial \xi_{i} \partial \xi_{j}}, & i, j=1, \ldots, n\end{cases}
$$

Тогда $U$ определяет изометрическое погружение $L^{n}(-1) \rightarrow L^{n+1}(-1)$ с $g, h-$ соответственно первой и второй квадратичной формой. 
3.2. Изометрические погружения пространственных форм в качестве подмногообразий отрицательной внешней кривизны. Локальное изометрическое вложение пространства Лобачевского $L^{l}$ в евклидово пространство $E^{2 l-1}$ было задано $\Phi$. Шуром [156]. Оно имеет вид:

$$
\begin{gathered}
x_{2 i-1}=a_{i} e^{y_{l}} \cos \left(y_{i} / a_{i}\right), \quad x_{2 i}=a_{i} e^{y_{l}} \sin \left(y_{i} / a_{i}\right), \quad 1 \leqslant i \leqslant l-1, \\
x_{2 l-1}=\int_{0}^{y_{l}}\left(1-e^{2 u}\right)^{1 / 2} d u,
\end{gathered}
$$

где $a_{i} \neq 0, \sum_{i=1}^{l-1} a_{i}^{2}=1$.

Это есть обобщение псевдосферы на многомерный случай. Э. Картаном в 1919 году было доказано, что пространства Лобачевского $L^{l}$ локально нельзя изометрично вложить в $E^{2 l-2}$ [44], [111].

Теорема 3.2.1 (Отзуки [127]). Риманово отрицательной секиионной кривизны нельзя локально изометрично вложить в $E^{2 n-2}$.

Доказательство теоремы сушественно опирается на сформулированную ниже леммy.

Условимся, что индексы будут пробегать следуюшие значения: $1 \leqslant i, j, k, l \leqslant n$; $1 \leqslant r \leqslant n-2 ; 1 \leqslant s \leqslant n-1 ; 1 \leqslant m \leqslant N ; 1 \leqslant \alpha, \beta, \gamma, \delta, \rho \leqslant 2 n-2$.

Пусть задана система квадратичных уравнений с $n$ неизвестными

$$
A_{i j}^{m} x^{i} x^{j}=0
$$

Обозначим $A(x, x)$ вектор с компонентами $A_{i j}^{m} x^{i} x^{j} ; A(x, y)$ - вектор с компонентами $A_{i j}^{m} x^{i} y^{j}$, где $x, y-n$-мерные векторы с компонентами $x^{i}, y^{i} ;\langle\cdot, \cdot\rangle-$ скалярное произведение в евклидовом пространстве.

Лемма 3.2.1. Если в системе $N$ уравнений (3.2.1) $N \leqslant n-1$ и для любых $x$ и у виполняется условие $\langle A(x, x), A(y, y)\rangle \leqslant\langle A(x, y), A(x, y)\rangle$, то система (3.2.1) имеет нетривиальное решение.

Для $n=3$ эту лемму доказали С. Черн и Н. Кейпер [48], для произвольного $n$ ее доказал Т. Отзуки [127].

ДокАЗАТЕЛЬСТво ТеОРемЫ 3.2.1. Пусть $z_{r}=A_{i j}^{r} t^{i} t^{j}$ есть $n$-мерная поверхность $F$ неположительной кривизны в $E^{2 n-2}$. Покажем, что в точке $t_{i}=0$ найдется такая двумерная площадка, в направлении которой кривизна равна нулю.

Пусть $x=\sum x^{i} e_{i}$ и $y=\sum y^{i} e_{i}$ - единичные взаимно-перпендикулярные векторы в касательном пространстве точки $O$ поверхности $F, e_{i}$ - единичные векторы направлений осей $t^{i}, n_{\alpha}$ - ортонормированный базис нормалей. Тогда кривизна $K(x, y)$ поверхности $F$ в направлении двумерной плошадки, натянутой на $x, y$, вычисляется по формуле

$$
K(x, y)=\left[A(x, x) A(y, y)-A^{2}(x, y)\right]
$$


Пусть $x_{0}=\sum x_{0}^{i} e_{i}$ - нетривиальное единичное решение системы $A_{i j}^{r} x^{i} x^{j}=0$; оно сушествует, поскольку система удовлетворяет условию леммы. Так как $A\left(x_{0}, x_{0}\right)=0$, из (3.2.2) следует, что

$$
K\left(x_{0}, y\right)=-A^{2}\left(x_{0}, y\right)
$$

Рассмотрим вектор $A\left(x_{0}, y\right)$, его компоненты $A_{i j}^{r} x_{0}^{i} y^{j}=0$. Направление вектора $x_{0}$ возьмем за направление оси $t^{n}$. Тогда, поскольку $\left(x_{0}, y\right)=0$, получим

$$
A_{i j}^{r} x_{0}^{i} y^{j}=A_{n s}^{r} y^{s}
$$

Введем вектор $A_{n s}$ с компонентами $A_{n s}^{r}$; тогда можно записать $A\left(x_{0}, y\right)=A_{n s} y^{s}$. Векторы $A_{n s}$ имеют размерность $n-2$, всего таких векторов $n-1$, значит, векторы $A_{n s}$ линейно зависимы. Поэтому можно подобрать $y_{0}^{s}$ такие, что $\sum y_{0}^{s}=1$ и $A_{n s} y_{0}^{s}=$ $A\left(x_{0}, y_{0}\right)=0$.

Из формулы (3.2.3) видно, что кривизна поверхности $F$ в направлении двумерной плошадки, натянутой на векторы $x_{0}, y_{0}$, равна нулю, чем утверждение теоремы доказано.

Используя этот результат, можно выяснить вопрос о непогружаемости риманова пространства $R^{n}$ в риманово пространство $R^{2 n-2}$ при некоторых ограничениях на кривизны пространств $R^{n}$ и $R^{2 n-2}$.

Пусть $R^{2 n-2}$ - риманово пространство, кривизна которого в каждом двумерном направлении $\geqslant c_{0}$, где $c_{0}$ - произвольная постоянная; обозначим его через $R_{c_{0}}^{2 n-2}$. Пусть $R^{n}$ - риманово пространство, кривизна которого в направлении каждой двумерной плошадки $\leqslant c_{0}-c^{2}, c>0$ (обозначим его через $R_{c_{0}-c^{2}}^{n}$ ), и пусть $P$ - произвольная точка $R_{c_{0}-c^{2}}^{n}, Q(P)$ - произвольная окрестность точки $P$ на $R_{c_{0}-c^{2}}^{n}$.

Как следствие теоремы 3.2.1 имеет место

TеОРема $3.2 .2([28])$. Окрестность $Q(P)$ точки $P$ пространства $R_{c_{0}-c^{2}}^{n}$ нельзя изометрично вложить в $R_{c_{0}}^{2 n-2}$.

ДокАЗАтЕльство. Пусть $z_{\rho}=z_{\rho}(x)$ есть $n$-мерная поверхность $R_{c_{0}-c^{2}}^{n}$ в $R_{c_{0}}^{2 n-2}$. Тогда

$$
R_{i j k l}=\left(A_{r i k} A_{r j l}-A_{r i l} A_{r j k}\right)+\bar{R}_{\alpha \beta \gamma \delta} z_{i}^{\alpha} z_{j}^{\beta} z_{k}^{\gamma} z_{l}^{\delta}
$$

где $R_{i j k l}$ - тензор кривизны $R_{c_{0}-c^{2}}^{n} ; \bar{R}_{\alpha \beta \gamma \delta}$ - тензор кривизны объемлющего пространства; $A_{r i j}$ - компоненты вторых квадратичных форм для $n-2$ взаимно перпендикулярных единичных нормалей; $z_{i}^{\alpha}=\frac{\partial z_{\alpha}}{\partial x_{i}}$.

В окрестности $R_{c_{0}-c^{2}}^{n}$ можно ввести такие координаты, что поверхность будет определяться уравнениями

$$
z_{n+r}=0, \quad z_{i}=x_{i}
$$

Тогда уравнения (3.2.4) примут вид

$$
R_{i j k l}=\sum_{r}\left(A_{i k}^{r} A_{j l}^{r}-A_{i l}^{r} A_{j k}^{r}\right)+\bar{R}_{i j k l} .
$$


Вычислим кривизну $R_{c_{0}-c^{2}}^{n}$ в точке $z_{\rho}=0$. Пусть $x, y$ - единичные взаимно перпендикулярные векторы, лежашие в касательном пространстве $R_{c_{0}-c^{2}}^{n}$ в точке $O$. Обозначим через $K(x, y), \bar{K}(x, y)$ кривизны $R_{c_{0}-c^{2}}^{n}, R_{c_{0}}^{2 n-2}$ в направлении двумерной плошадки, натянутой на векторы $x, y$. Из (3.2.5) получим

$$
K(x, y)=\langle A(x, x), A(y, y)\rangle-\langle A(x, y), A(x, y)\rangle+\bar{K}(x, y) .
$$

По условию теоремы для взаимно перпендикулярных единичных векторов $K(x, y) \leqslant c_{0}-c^{2}, \bar{K}(x, y) \geqslant c_{0}$; из (3.2.6) находим

$$
\langle A(x, x), A(y, y)\rangle-\langle A(x, y), A(x, y)\rangle \leqslant-c^{2} .
$$

Но координаты $x^{i}$ выбраны так, что компоненты метрического тензора пространства $R_{c_{0}-c^{2}}^{n}$ в точке $O$ будут $g_{i j}=\delta_{i j}$; поэтому

$$
\sum x_{i}^{2}=1, \quad \sum y_{i}^{2}=1
$$

И если теперь в $E^{2 n-2}$ задать поверхность уравнением $z^{r}=A_{i j}^{r} x_{i} x_{j}$, то из (3.2.7) и (3.2.8) следует, что она в точке $O$ будет иметь строго отрицательную кривизну; это противоречит теореме 3.2.1. Этим и доказана теорема 3.2.2.

Изучим локальные свойства изометрического вложения $L^{l}$ в $E^{2 l-1}$.

Пусть $V$ - $n$-мерное векторное пространство надполем вещественных чисел, $\Phi^{1}, \Phi^{2}$, $\ldots, \Phi^{n}$ - симметрические билинейные формы на $V$. Будем говорить, что $\Phi^{1}, \ldots, \Phi^{n}$ внешне ортогональны, если

$$
\sum_{\lambda=1}^{n}\left[\Phi^{\lambda}(x, y) \Phi^{\lambda}(z, w)-\Phi^{\lambda}(x, w) \Phi^{\lambda}(z, y)\right]=0
$$

для $x, y, z, w \in V$.

Теорема 3.2 .3 (Картан [44]). Предположим, ито $\Phi^{1}, \ldots, \Phi^{n}$ суть внешне ортогональные симметричные билинейнье формы на вещественном векторном пространстве $V$.

Пусть виполняется условие: если вектор $X \in V$ таков, что $\Phi^{\lambda}(x, y)=0$ для $1 \leqslant \lambda \leqslant n$ и для всех $y \in V$, то $X=0$.

Тогда существуют вещественная ортогональная матрица $\left(a_{\mu}^{\lambda}\right)$ и $n$ линейных функиионалов $\varphi^{1}, \ldots, \varphi^{n}$ такие, что

$$
\Phi^{\lambda}=\sum_{\mu} a_{\mu}^{\lambda} \varphi^{\mu} \otimes \varphi^{\mu}, \quad 1 \leqslant \lambda \leqslant n .
$$

Это значит, что $\Phi^{1}, \ldots, \Phi^{n}$ одновременно диагонализуемы относительно базиса, дуального к $\varphi^{1}, \ldots, \varphi^{n}$.

Пусть $M$ есть $l$-мерное риманово многообразие постоянной секционной кривизны $k$, изометрично вложенное в риманово многообразие постоянной кривизны $K$, и пусть 
$k<K$. Если мы рассмотрим вторые квадратичные формы $A^{\lambda}(x, y)$ относительно ортонормированного базиса нормалей и форму $\Psi=\sqrt{K-k} d s^{2}$, где $d s^{2}-$ первая квадратичная форма, то из формулы Гаусса следует, что формы $A^{1}(x, y), \ldots, A^{l-1}(x, y)$, $\Psi(x, y)$ внешне ортогональны. Тогда из теоремы 3.2 .3 следует, что существует ортонормированньй базис $e_{1}, \ldots, e_{l}$ в каждом касательном пространстве $T_{Q} M$, в котором первая и вторая квадратичные формы одновременно приводятся к диагональному виду. Эти направления называются направлениями кривизны или главньми направлениями. Линии, касаюшиеся главных направлений, назьваются линиями кривизны. Асимптотическим вектором $X \in T_{Q} M$ называется вектор, для которого $A^{\lambda}(x, x)=0$ одновременно для всех вторых квадратичных форм. Из теоремы Безу и леммы 3.2.1 следует, что существует $2^{l}$ асимптотических направлений, причем они имеют форму

$$
\pm x^{1} e_{1}+ \pm x^{l} e_{l}
$$

т.е. асимптотические векторы направлены по диагоналям куба, натянутого на главные направления.

Д. Мур показал, что асимптотические линии можно выбрать в качестве координатных линий. В асимптотических координатах линейный элемент имеет вид

$$
d s^{2}=\sum_{i=1}^{l} d u_{i}^{2}+2 \sum_{i \neq j} \cos \omega_{i j} d u_{i} d u_{j}
$$

т.е. координатная сеть обладает чебьшёвским свойством [117]. Ю.А. Аминов показал, что существует система координат, в которой координатные линии являются линиями кривизны [7], [9], [10] и линейный элемент для изометрического погружения пространства Лобачевского $L^{l}$ в евклидово пространство $E^{2 l-1}$ записьвается в виде

$$
d s^{2}=\sum_{i=1}^{n} \sin ^{2} \sigma_{i} d u_{i}^{2}
$$

причем $\sum_{i=1}^{n} \sin ^{2} \sigma_{i}=1$.

Заметим, что системы ортогональных координат в $n$-мерном евклидовом пространстве с условием постоянства суммы метрических коэффициентов рассматривались первоначально К. Гишаром, затем Г. Дарбу и Л. Бианки. В [7] доказано, что уравнения Кодацши-Риччи имеют своим условием интегрируемости уравнения Гаусса. Поэтому вся система уравнений погружения $L^{n}$ в $E^{2 n-1}$ сводится к системе

$$
R_{i j i j}=\sin ^{2} \sigma_{i} \sin ^{2} \sigma_{j}, \quad R_{i j k j}=0, \quad i \neq k, \quad \sum_{i=1}^{n} \sin ^{2} \sigma_{i}=1
$$

Доказано, что для построения произвольного локального погружения $L^{n}$ в $E^{2 n-1}$ необходимо и достаточно указать способ построения в $L^{n}$ системы ортогональных координат с условием $\sum_{i=1}^{n} \sin ^{2} \sigma_{i}=1$, что приводит к системе (3.2.9). Система (3.2.9) является многомерным аналогом уравнения "синус Гордона" $\omega_{x x}-\omega_{y y}=\sin \omega$ или, в другой системе координат, $\omega_{x y}=\sin \omega$. 
Э. Картан показал, что аналитические изометрические вложения пространства Лобачевского $E^{n}$ в $E^{2 n-1}$ имеют произвол в $n(n-1)$ функций одного переменного [45], [78].

Свойства изометрического погружения пространства Лобачевского $L^{l}$ в $E^{2 l-1}$ изучались в цикле работ Ю.А. Аминовым. В частности, если погружение $L^{3}$ в $E^{5}$ удовлетворяет некоторому геометрическому условию, то основная система погружения содержит в себе в качестве подсистемы уравнение движения твердого тела вокруг его центра масс в нњютоновом поле тяготения [11].

Система дифференциальных уравнений изометрического погружения $L^{4}$ в $E^{7}$ aсcоциируется с системой уравнений Максвелла. Естественньм образом вводится тензор электромагнитной напряженности.

Изучался также грассманов образ $L^{l}$ в $E^{n}$. Если каждой точке $P \in L^{l} \subset E^{n}$ поставить в соответствие нормальное пространство к $L^{l}$ в этой точке, параллельно перенесенное в фиксированную точку $E^{n}$, то мы получим грассманово отображение $L^{l}$ в грассманово многообразие $(n-l)$-мерных плоскостей $G(n-l, n)$, образ отображения обозначим через $\Gamma$. Метрика грассманова образа $\Gamma_{2}$ пространства Лобачевского $L^{l}$ в $E^{2 l-1}$ имеет вид:

$$
d \sigma^{2}=\sum_{i=1}^{n} \cos ^{2} \sigma_{i} d u^{2} .
$$

Для пространства Лобачевского $L^{l}$ в $E^{2 l-1}$ секционная кривизна грассманова образа $G(l-1,2 l-1)$ по двумерным плошадкам, касательным к грассманову образу $\Gamma$, лежит в интервале $(0,1)[7]$. Заметим, что кривизна грассманова многообразия меняется в пределах $[0,2]$.

ТеОРема 3.2.4 (Аминов [12]). Если ds ${ }^{2}$ - метрика пространства Лобачевского $L^{l}$ и d $\sigma^{2}$-метрика грассманова образа изометрического погружения области $L^{l}$ в $E^{n}$ с плоской нормальной связностью, то метрика

$$
d s^{2}+d \sigma^{2}
$$

плоская.

ТеОРема 3.2 .5 (Аминов [13]). Не существует локального класса $C^{3}$-изометрического погружения $L^{3}$ в $E^{5}$ с постоянной секчионной кривизной метрики грассманова образа.

Усилия многих авторов были направлены на обобшение теоремы Гильберта на многомерный случай. При различных дополнительных условиях были получены результаты в этом направлении.

ТеОРема 3.2.6 (Аминов [9]). Не существует погружения всего пространства Лобачевского, при котором линии кривизны одного семейства геодезические.

Теорема 3.2.7 (Аминов [11]). Не существует регулярного класса $C^{3}$-изометрического погружения полного пространства Лобачевского $L^{3}$ в $E^{5}$ с семейством вполне геодезических поверхностей кривизны.

В [117] Д. Муром доказано, что не сушествует изометрического погружения полного пространства Лобачевского $L^{l}$ в $E^{2 l-1}$ в качестве минимального подмногообразия. 
В [115] это обобщено на изометрические погружения $L^{l}$ в $E^{l+\rho}(\rho \geqslant l-1)$ с плоской нормальной связностью. Заметим, что при $\rho=l-1$ изометрическое погружение $L^{l}$ в $E^{2 l-1}$ имеет плоскую нормальную связность.

Если изометрически погружаем не полное односвязное пространство Лобачевского, а гиперболические многообразия (полные неодносвязные римановы многообразия постоянной отрицательной секционной кривизны), то в этом случае получены, в некотором смысле, окончательные результаты: в [176] Ф. Ксавье доказана невозможность изометрического погружения гиперболического многообразия $H^{l}$ в $E^{2 l-1}$, если $H^{l}$ имеет неэлементарную фундаментальную групу (т.е. фундаментальная групша имеет бесконечное предельное множество на абсолюте). Это эквивалентно тому, что фундаментальная группа имеет, по крайней мере, две образуюшие.

Когда $H^{l}$ - фуксово многообразие, этот результат обобшался в [128]. Наибольшее продвижение дает

ТЕорема 3.2.8 (Николаевский [124]). Полное неодносвязное гиперболическое многообразие $H^{l}$ не допускает регулярного изометрического погружения в $E^{n}$ с плоской нормальной связностью.

СлЕДСтвИЕ. Полное неодносвязное гиперболическое многообразие $H^{l}$ не допускает регулярного изометрического погружения в $E^{2 n-1}$.

Это следует из того факта, что изометрическое погружение $H^{l}$ в $E^{2 l-1}$ имеет плоскую нормальную связность.

ДоКАЗАТЕЛЬСТво ТЕОРемЫ 3.2.8. Пусть $f: H^{l} \rightarrow E^{n}$ - изометрическое погружение $H^{l}$ с плоской нормальной связностью. Из у равнений Риччи следует, что в каждой точке $F^{l}=f\left(H^{l}\right)$ сушествуют главные направления. Из [7] следует, что эти направления голономны и линии кривизны можно выбрать за координатные линии и локально метрика $g$ на $F^{l}$ в этих координатах имеет вид

$$
d s^{2}=\sum_{i=1}^{l} \sin ^{2} \sigma_{i}\left(d u_{i}\right)^{2},
$$

метрика грассманова образа $F^{l}$ имеет вид

$$
d \sigma^{2}=\sum \cos ^{2} \sigma_{i} d u_{i}^{2}
$$

Определим на $H^{l}$ плоскую метрику $g_{0}$,

$$
d s_{0}^{2}=\sum_{i=1}^{l}\left(d u_{i}\right)^{2},
$$

которая локально является плоской. Возьмем универсальную накрьвающую $\widetilde{H}^{l}$ многообразия $H^{l}$, которая гомеоморфна евклидову пространству $E^{n}$.

Поднимем метрики $g$ и $g_{0}$ на $E^{n}$. Метрика $g_{0}$ также полная. Это следует из полноты метрики $g$ и того, что длины соответствуюших кривых в метрике $g_{0}$ больше, чем в метрике $g$. В поднятой метрике $g_{0} E^{n}$ глобально изометрично евклидову пространству. Тем самым мы имеем на $E^{n}$ две метрики: гиперболическую метрику $g$ и 
евклидову $g_{0}$. Действие фундаментальной групшы $\pi_{1}\left(H^{l}\right)$ сохраняет первую и вторую квадратичные формы $F^{l}=f\left(H^{l}\right)$, а значит, и метрику грассманова образа. Поэтому $\pi_{i}\left(H^{l}\right)$ является одновременно изометрией на $E^{n}$ для метрик $g$ и $g_{0}$ и переводит координатные линии $u_{i}$ в себя. Пусть $\gamma$ - нетривиальный элемент $\pi_{i}\left(H^{l}\right), u\left(u_{1}, \ldots, u_{l}\right)-$ координаты. Тогда $\gamma(u)=A u+b$, где $A$ - ортогональная матрица, состояшая только из $0, \pm 1$.

Сушествует $m$ такое, что $A^{m}$ есть тождественная матрица. Тогда $\Phi(u)=\gamma^{m}(u)=$ $u+c$, где $c\left(c^{1}, \ldots, c^{l}\right)=$ const, $c \neq 0$, так как в противном случае $\gamma$ имеет фиксированную точку и $H^{l}=E^{l} / \pi_{i}\left(H^{l}\right)$ не является многообразием. Изометрия $\Phi=\gamma^{m}$ метрики $g_{0}$ является параллельным переносом и $\operatorname{dist}(u, \Phi(u))=\|c\|=$ const. $\mathrm{C}$ другой стороны, изометрия $\Phi$ метрики $g<g_{0}$ обладает свойством $\operatorname{dist}(u, \Phi(u))<$ $\operatorname{dist}_{0}(u, \Phi(u))=$ const. Но хорошо известно, что изометрия пространства Лобачевского с таким свойством является тождественным преобразованием. Мы пришли к противоречию.

Если объемлющим пространством являются другие пространственные формы, то имеет место

ТЕОРема 3.2 .9 ([31]). Пусть $F^{l}$ - компактная l-мерная поверхность постоянной секционной кривизны с в пространстве постоянной кривизны $C$. Если $c<C$, то $C>0, c=0$ и поверхность локально изометрична евклидову пространству.

ДокАЗАТЕльство. Невозможность случая $0<c<C$ доказанав [117]. На $F^{l}$ есть поле главных направлений, которое голономно. Сушествует конечнократноекомпактное параллелизуемое накрьвающее многообразие $M^{l}$. Параллелизацию $M^{l}$ образуют векторные поля главных направлений. В окрестности каждой точки многообразия $M^{l}$ интегральные траектории векторных полей, которые параллелизуют многообразие в силу голономности, можно брать за координатные линии параллелизации. Отсюда следует, что на $M^{l}$ можно ввести локально евклидову метрику. Накрьвающим пространством для компактного локально евклидова многообразия является тор $T^{l}$. Он является накрьвающим пространством и для подмногообразия $F^{l}$. Тогда на торе индуцируется метрика постоянной кривизны $C$. Но известно, что если на торе $T^{l}$ задана риманова метрика знакопостоянной секционной кривизны, то она плоская [108]. Отсюда следует, что $c=0$. В силу условия теоремы $C>0$.

В [31] использовалось поле асимптотических направлений.

Изометрические вложения пространства Лобачевского $L^{l}, l \geqslant 3$, в гильбертово пространство построил Д. Блануша [18]. Первые вложения в конечномерное пространство $E^{n}$ были построены в [23] для $l \geqslant 3, n=6 l-5$ и для $l=2, n=6$, причем вложения являются $C^{\infty}$-гладкими и задаются явными формулами. Из теорем Нэша и Громова следует более высокая оценка на $n$.

В [87] В. Хенке были заданы $C^{\infty}$-изометричные погружения пространства Лобачевского $L^{l}(C)$ кривизны $C$ в сферическое пространство кривизны $\widetilde{C}$, если $C<0<\widetilde{C}$; и $L^{l}(C) \rightarrow L^{4 n-3}(\widetilde{C})$, если $C<\widetilde{C}<0$.

Пусть $M^{l}\left(\widetilde{M}^{n}\right)$-пространственные формы постоянной кривизны, причем $n \geqslant 4 l-3$. Если $M^{l}$ односвязно, то сушествует $C^{\infty}$-изометрическое погружение $M^{l}$ в $M^{n}$. 
Изометрическое погружение пространства Лобачевского $L^{l}$ в $E^{4 l-3}$ было дано В $[86]$.

В [14] Д.Г. Азовым рассмотрено два класса римановых метрик

$$
\begin{gathered}
d s^{2}=d u_{1}^{2}+f\left(u_{1}\right) \sum_{i=2}^{l} d u_{i}, \quad f>0 \\
d s^{2}=g^{2}\left(u_{1}\right) \sum_{i=2}^{l} d u_{i}^{2}, \quad g>0
\end{gathered}
$$

и решен вопрос об их погружаемости в $E^{n}$ и $S^{n}, n>l$. Функции $f$ и $g$ будем считать принадлежашими классу $C^{1}$. В частном случае при $g\left(u_{1}\right)=\frac{1}{u_{1}}, u_{1}>0$, получаем метрику пространства Лобачевского. Доказаны следуюшие теоремы.

Теорема 3.2.10 (Азов [14]). Метрики (3.2.10) и (3.2.11) допускают изометрические погружения в евклидово пространство $E^{4 l-3}$.

Теорема 3.2.11 (Азов [14]). Метрики (3.2.10) и (3.2.11) допускают погружения в сферическое пространство $S^{4 l-3}$.

Доказательства этих утверждений проведены методами, аналогичными предложенным в работах Д. Блануши; при этом даны конкретные параметрические уравнения поверхностей. Класс гладкости этих поверхностей будет совпадать с классом гладкости функций $f$ и $g$.

Введем, следуя Д. Блануше [23], ряд вспомогательных функций. Пусть

$$
\begin{gathered}
A=\int_{0}^{1} \sin \pi \xi e^{-\frac{1}{\sin ^{2} \pi \xi}} d \xi \\
\varphi_{1}(u)=\left(\frac{1}{A} \int_{0}^{u+1} \sin \pi \xi e^{-\frac{1}{\sin ^{2} \pi \xi}} d \xi\right)^{1 / 2}, \quad \varphi_{2}(u)=\left(\frac{1}{A} \int_{0}^{u} \sin \pi \xi e^{-\frac{1}{\sin ^{2} \pi \xi}} d \xi\right)^{1 / 2}
\end{gathered}
$$

Введем следуюшие обозначения: $a=\max \left(\left|\varphi_{1}^{\prime}\right|,\left|\varphi_{2}^{\prime}\right|\right)$,

$$
\begin{aligned}
& \psi_{1, f}(u)=2(a+1)\left\{\left[\max _{2 k-1 \leqslant u \leqslant 2 k+1}\left(|f|,\left|f^{\prime}\right|\right)\right]+1\right\}, \quad 2 k-1 \leqslant u<2 k+1, \\
& \psi_{2, f}(u)=2(a+1)\left\{\left[\max _{2 k-2 \leqslant u \leqslant 2 k}\left(|f|,\left|f^{\prime}\right|\right)\right]+1\right\}, \quad 2 k-2 \leqslant u<2 k .
\end{aligned}
$$

Здесь квадратные скобки обозначают целую часть стоящего в них выражения. Пусть

$$
\begin{gathered}
h_{1, f}=\frac{f(u) \varphi_{1}(u)}{\psi_{1, f}(u)}, \quad h_{2, f}=\frac{f(u) \varphi_{2}(u)}{\psi_{2, f}(u)} \\
\alpha_{f}(u, t)=\sqrt{l-1} \cdot t \psi_{1, f}(u), \quad \beta_{f}(u, t)=\sqrt{l-1} \cdot t \psi_{2, f}(u) .
\end{gathered}
$$


Тогда погружение метрики (3.2.10) в евклидово пространство $E^{4 l-3}$ может быть дано следуюшими параметрическими уравнениями:

$$
\begin{gathered}
x_{0}=\int_{0}^{u_{1}} \sqrt{1-h_{1, f}^{\prime 2}(\xi)-h_{2, f}^{\prime 2}(\xi)} d \xi \\
x_{i 1}=\frac{h_{1, f}\left(u_{1}\right)}{\sqrt{l-1}} \cos \alpha_{f}\left(u_{1}, u_{i}\right), \quad x_{i 2}=\frac{h_{1, f}\left(u_{1}\right)}{\sqrt{l-1}} \sin \alpha_{f}\left(u_{1}, u_{i}\right), \\
x_{i 3}=\frac{h_{2, f}\left(u_{1}\right)}{\sqrt{l-1}} \cos \beta_{f}\left(u_{1}, u_{i}\right), \quad x_{i 4}=\frac{h_{2, f}\left(u_{1}\right)}{\sqrt{l-1}} \sin \beta_{f}\left(u_{1}, u_{i}\right), \quad i=2, \ldots, l .
\end{gathered}
$$

Введем функцию

$$
\Phi(u)=\int_{0}^{u} \frac{\left[\left(1-h_{1, f}^{\prime 2}-h_{2, f}^{\prime 2}\right)\left(1-h_{1, f}^{2}-h_{2, f}^{2}\right)-\left(h_{1, f}^{\prime} h_{1, f}+h_{2, f}^{\prime} h_{2, f}\right)^{2}\right]^{1 / 2}}{1-h_{1, f}^{2}-h_{2, f}^{2}} d \xi
$$

где $h_{j, f}=h_{j, f}(\xi), j=1,2$.

Погружение метрики (3.2.10) в сферическое пространство $S^{4 l-3}$ может быть дано следуюшими уравнениями:

$$
\begin{gathered}
x_{01}=\sqrt{1-h_{1, f}^{2}\left(u_{1}\right)-h_{2, f}^{2}\left(u_{1}\right)} \cdot \cos \Phi\left(u_{1}\right), \\
x_{02}=\sqrt{1-h_{1, f}^{2}\left(u_{1}\right)-h_{2, f}^{2}\left(u_{1}\right)} \cdot \sin \Phi\left(u_{1}\right), \\
x_{i 1}=\frac{h_{1, f}\left(u_{1}\right)}{\sqrt{l-1}} \cos \alpha_{f}\left(u_{1}, u_{i}\right), x_{i 2}=\frac{h_{1, f}\left(u_{1}\right)}{\sqrt{l-1}} \sin \alpha_{f}\left(u_{1}, u_{i}\right), \\
x_{i 3}=\frac{h_{1, f}\left(u_{1}\right)}{\sqrt{l-1}} \cos \beta_{f}\left(u_{1}, u_{i}\right), \quad x_{i 4}=\frac{h_{1, f}\left(u_{1}\right)}{\sqrt{l-1}} \sin \beta_{f}\left(u_{1}, u_{i}\right), \quad i=2, \ldots, l .
\end{gathered}
$$

Для метрики (3.2.11) доказательство теорем 3.2.10 и 3.2.11 проводится совершенно аналогично. Нужно только следуюшим образом изменить ступенчатые функции $\psi_{1}$ и $\psi_{2}$ :

$$
\begin{aligned}
& \psi_{1, g}=2(a+1)\left\{\left[\max _{2 k-1 \leqslant u \leqslant 2 k+1}\left(1,\left|\frac{g^{\prime}}{g}\right|\right)\right]+1\right\}, 2 k-1 \leqslant u \leqslant 2 k+1, \\
& \psi_{2, g}=2(a+1)\left\{\left[\max _{2 k-2 \leqslant u \leqslant 2 k}\left(1,\left|\frac{g^{\prime}}{g}\right|\right)\right]+1\right\}, \quad 2 k-2 \leqslant u \leqslant 2 k .
\end{aligned}
$$

Кроме того, нужно заменить функцию $1-h_{1, f}^{\prime 2}-h_{2, f}^{\prime 2}$ функцией $g^{2}-h_{1, g}^{\prime 2}-h_{2, g}^{\prime 2}$.

3.3. Изометрические погружения в виде подмногообразий положительной внешней кривизны.

3.3.1. $k$-омбилические подмногообразия. Известно, что регулярная гиперповерхность $F^{l}(c), l \geqslant 3$, постоянной секционной кривизны $c$ в пространстве постоянной секционной кривизны $M^{l+1}(\widetilde{c})$ локально обладает следующими свойствами:

1) либо $c=\widetilde{c}$ и внешний нуль-индекс $\mu \geqslant l-1$;

2) либо $c>\widetilde{c}$ и гиперповерхность является омбилической, т.е. все главные кривизны равны и постоянны [58], [159]. 
Омбилическими гиперповерхностями в сфрерическом евклидовом пространстве являются сферы, в пространстве Лобачевского - сферы, эквидистантные гиперповерхности, орисферы.

При изометрическом погружении пространств $M^{l}(c)$ постоянной кривизны $c$ в пространства постоянной кривизны $M^{l+p}(\widetilde{c})$, где $c>\widetilde{c}$, при достаточно малой коразмерности $p<l$ наследуются некоторые омбилические свойства гиперповерхностей.

Точка $P$ подмногообразия $M^{l}(c) \subset M^{l+p}(\widetilde{c}), c>\widetilde{c}$, назьвается изотропной, если векторы нормальной кривизны $A(x, x)$ во всех направлениях равны между собой.

В [126] Б. О'Нейлом показано, что при $p \leqslant l-2$ в каждом касательном пространстве $T_{Q} M^{l}(c), Q \in M^{l}(\widetilde{c})$, существует изотропное подпространство $L(Q)$ размерности $\rho(Q) \geqslant l-p+1$ (векторы нормальной кривизны в направлениях, которые лежат в этом подпространстве, равны между собой). Если распределение $L(Q)$ и поля векторов нормальной кривизны регулярны, то распределение $L(Q)$ интегрируемо и каждьй слой является изотропньм подмногообразием в $M^{l+p}(\widetilde{c})[126]$.

Для подмногообразий с кривизной, равной $c>0$, справедлив глобальньй результат:

ТеОрема 3.3 .1 (О'Нейл [126]). Пусть $M^{l}(c), c>0$, есть полное регулярное подмногообразие в $M^{l+2}(\widetilde{c}), l \geqslant 4, c>\widetilde{c}$. Тогда на $M^{l}(c)$ есть изотропная точка.

Теорема неверна, если $c \leqslant 0$ или $p>2$. Для случая $c<\widetilde{c}$ и коразмерности $p=l-1$ из теоремы 3.2 .3 следовало, что вторые квадратичные формы подмногообразия ортогонально диагонализуемы, т.е. первая и вторые квадратичные формы одновременно приводятся к диагональному виду. Для случая $c>\widetilde{c}$ имеет место двойственньй результат.

Лемма 3.3.1 (Мур [118]). Пусть $c-\widetilde{c}=k>0 u p \leqslant l-1$. Eсли в точке $P \in M^{l}(c) \subset M^{l+p}(\widetilde{c})$ существует единичный вектор $x \in T_{Q} M^{l}$ такой, что квадрат длинь вектора нормальной кривизньи $\langle A(x, x), A(x, x)\rangle<k$, то $p=l-1$ и вторая квадратичная форма $M^{l}(c)$ ортогонально диагонализуема.

ОПРЕДЕЛЕНИЕ. Точка $P \in M^{l}(c) \subset M^{l+p}(\widetilde{c}), c-\widetilde{c}=k>0$, называется сильно омбилической, если существует единичньй нормальньй вектор $n \in N_{P} M^{l}$ такой, что вторая квадратичная форма имеет вид

$$
A(x, y)=\sqrt{k}\langle x, y\rangle n
$$

для $x, y \in T_{P} M^{l}$.

Подмногообразия из сильно омбилических точек в сфферическом и евклидовом пространствах являются сферами в некотором вполне геодезическом $M^{l+1}(\widetilde{c})$; в пространстве Лобачевского - сферами, эквидистантньми поверхностями, орисферами в некотором вполне геодезическом пространстве. Если $M^{l}(c)$ есть полное регулярное подмногообразие в $M^{l+2}(\widetilde{c}), c>0, c-\widetilde{c}>0$, то $M^{l}(c)$ имеет сильно омбилические точки [126]. Результат не имеет места при $c<0$ и $p>2$.

ОПреДЕЛЕНИЕ. Точка $P \in M^{l}(c) \subset M^{l+p}(\widetilde{c}), c-\widetilde{c}=k>0$, назьвается слабо омбилической, если существует единичньй нормальньй вектор $n \in N_{P} M^{l}$ такой, что вторая квадратичная форма $A$ удовлетворяет уравнению

$$
\langle A(x, y), n\rangle=\sqrt{k}\langle x, y\rangle
$$

для $x, y \in T_{P} M$. 
Имеет место

Лемма 3.3.2 (Мур [118]). Пусть $c-\widetilde{c}=k>0 u p \leqslant l-1$. Если длина векторов нормальной кривизны $\langle A(x, x), A(x, x)\rangle \geqslant k$, для всех $x \in T_{P} M^{l}$, то точка $P \in M^{l}$ является слабо омбилической.

Из лемм 3.3.1, 3.3.2 следует, что при $p \leqslant l-2$ каждая точка $M^{l}$ является слабо омбилической; при $p=l-1$ либо является слабо омбилической, либо вторые квадратичные формы ортогонально диагонализуются. Однако в глобальном случае справедлива

Tеорема 3.3.2 (Myp [118]). Пусть $c-\widetilde{c}>0 u c>0 . E c \Omega u M^{l}(c) \subset M^{2 l-1}(\widetilde{c})$ есть полное регулярное подмногообразие постоянной положсительной кривизнь, то каждая точка $M^{l}(c)$ является слабо омбилической.

Для подмногообразий $F^{l}$ в пространствах постоянной кривизны $M^{l+p}(\widetilde{c})$ можно определить индекс сильной омбиличности $s(P)$. В точке $P \in F^{l}$ это размерность максимального подпространства $L(P) \subset T_{P} F^{l}$ такого, что ограничение второй квадратичной формы $F^{l}$ на $L(P)$ имеет вид

$$
A(x, y)=\sqrt{k}\langle x, y\rangle n
$$

для любых $x \in L(P), y \in T_{P} F^{l}$.

Если $F^{l}$ изометрично $M^{l}(c), k=c-\widetilde{c}>0$, то индекс сильной омбиличности $s(Q) \geqslant$ $l-p+1, Q \in M^{l}(c)$, если $p \leqslant l-2$, и также при $p=l-1$, если точка слабо омбилична. Действительно, если в точке $Q \in M^{l}(c)$ возьмем ортонормированный базис нормалей $n_{1}, \ldots, n_{p-1}, n_{p}=n$, где $n-$ единичньй нормальньй вектор из леммы 3.3.2, то для вторых квадратичных форм относительно нормалей $n_{i}(i=1, \ldots, p-1)$ из формулы Гаусса следует равенство

$$
\sum_{\alpha=1}^{p-1} A^{\alpha}(x, x) A^{\alpha}(y, y)-A^{\alpha}(x, y) A^{\alpha}(x, y)=0
$$

т.е. внешний нуль-индекс $M^{l}(c)$, ограниченньй на подпространство нормалей, ортогональных $n$, удовлетворяет неравенству $\mu(Q) \geqslant l-p+1$. Напомним, что $\mu(Q)$ есть размерность максимального подпространства в $L(Q) \subset T_{Q} M^{l}$ такого, что $A(x, y)=0$ для $x \in L(Q), y \in T_{Q} M^{l}$. Ограничение вторых квадратичных форм $M^{l}(Q)$ на $L(Q)$ удовлетворяет (3.3.3) и индекс сильной омбиличности $s(Q)=\mu(Q) \geqslant l-p+1$. Пользуясь свойствами индекса изотропности [120], легко показать, что для $M^{l}(c) \subset M^{l+p}(\widetilde{c})$, $c-\widetilde{c}>0 \rho(Q)=s(Q)$ и максимальное пространство изотропности совпадает с максимальным пространством сильной омбиличности. Заметим, что сильная $k$-омбиличность является частным случаем сильной $k$-выпуклости [37].

Если распределение $L(Q)$ регулярно и векторное нормальное поле $n(Q)$ регулярно и параллельно в нормальном расслоении, то распределение $L(Q)$ инволютивно и слои являются омбилическими поверхностями в $M^{l}(c)$ и объемлющем пространстве $M^{l+p}(\widetilde{c})$. Если в точке $P$ индекс сильной омбиличности минимален, то распределение $L(Q)$ регулярно в окрестности точки $P$. Если нормальное пространство имеет постоянную размерность, то нормальное векторное поле $n$ регулярно. Из сильной омбиличности следует 
ТеОРема 3.3 .3 (Мур [118]). Пусть $M^{l}$ - компактное риманово многообразие постоянной секционной кривизны $c>0$. Пусть $M^{l}$ изометрично регулярно погружсено в евклидово пространство $E^{l+p}$, где $p \leqslant l / 2$. Тогда $M^{l}$ односвязно и изометрично стандартной сфере.

Евклидово пространство можно заменить односвязным пространством $M^{l+p}(\widetilde{c})$, $c>\widetilde{c}$. Частные результаты для коразмерности два получены в [85].

3.3.2. Продолжение изометрических погружений. В работах В. Хенке [84], [85] и Д. Эрбахера [66] изучены изометрические погружения $l$-мерной сферы в евклидово пространство $E^{l+2}$. Легко построить такие погружения, если рассмотреть погружение $E^{l+1}$ (или пространственной окрестности $S^{l}$ ) в $E^{l+2}$ (например, наматывая на цилиндр или конус) и это отображение ограничить на $S^{l}$. Ставится вопрос, может ли произвольное заданное изометрическое $C^{\infty}$-погружение $f: S^{l} \rightarrow E^{l+2}$ рассматриваться как ограничение изометрического $C^{\infty}$-погружения $E^{l+1}$ в $E^{l+2}$. Если отображение $f$ есть ограничение на $S^{l}$ некоторого изометрического отображения $F: E^{l+1} \rightarrow E^{l+2}$, то $f$ назьвается продолжимым, а отображение $F$ назьвается изометрическим продолжением $f$. Другими словами, это значит, что локальное (илиглобальное) изометрическое погружение $f$ стандартной сферы $S^{l}$ в $E^{l+2}$ является композицией $f=h \circ g$ стандартного вложения $g: S^{l} \rightarrow E^{l+1}$ и изометрического погружения $h: E^{l+1} \rightarrow E^{l+2}$. Оказывается, существование такого продолжения в некоторую окрестность точки $x \in S^{l}$ зависит от того, будет точка $x$ граничной точкой множества омбилических точек погружения $f$ или нет.

Аналогичные вопросы для изометрического погружения $E^{2}$ в $E^{4}$ рассматривались в главе 2. В работе [66] Д. Эрбахером доказано существование изометрического продолжения $F$ отображения $f: S^{l} \rightarrow E^{l+2}, l \geqslant 4$, в окрестности неомбилической точки $x \in S^{l}$. В [85] В. Хенке доказьвается единственность такого продолжения, если $x$ является неомбилической точкой, т.е. для этих точек имеет место некоторьй вид “жесткости". Если точка $x$ является внутренней точкой множества омбилических точек, то локальное изометрическое продолжение $f$ в окрестности $x$ также сушествует, но оно не единственно; напротив, сушествуют два определенных в окрестности $p$ локальных изометрических продолжения $f$, которые ни в одной окрестности не совпадают. Из этих утверждений следует, что в случае $l \geqslant 4$ локальное изометрическое погружение $S^{l} \rightarrow E^{l+2}$ имеет продолжение во всех точках $S^{l}$, за исключением граничного множества всех омбилических точек. В работе [85] построен пример такого изометрического $C^{\infty}$-погружения $f: S^{l} \rightarrow E^{l+1}$, что сушествует точка $p \in S^{l}$, в окрестности которой $f$ не является локально изометрически продолжимым. Изучено также геометрическое строение множества неомбилических точек $Z$. Существуют две аффинные гиперплоскости $H_{1}$ и $H_{2} \subset E^{l+1}$ с $H_{1} \cap S^{l} \neq \varnothing, H_{2} \cap S^{l} \neq \varnothing$ и $H_{1} \cap H_{2} \cap S^{l} \neq \varnothing$ такие, что открытые полупространства $H_{1}^{+}$и $H_{2}^{+}$в пересечении с $S^{l}$ дают $Z$, т.е. $Z=H_{1}^{+} \cap H_{2}^{+} \cap S^{l}$.

На вопрос о глобальном изометрическом продолжении отвечает

Теорема 3.3 .4 (Мур [120]). Пусть $S^{l}$ есть стандартная сфера кривизнь единица, рассматриваемая как граница единичного шара $D^{l+1}$. Eсли $l \geqslant 3$ и $f: S^{l} \rightarrow E^{l+2}$ есть $C^{\infty}$-изометрическое погружение, то существует единственное $C^{\infty}$-изометрическое погружение $\widetilde{f}: D^{l+1} \rightarrow E^{l+2}$ такое, что $\widetilde{f} / S^{l}=f$.

Теорема не верна, если $C^{\infty}$ заменить $C^{k}$. Пусть $\tilde{f}: E^{n+1} \rightarrow E^{n+2}$, заданное в виде 
$\widetilde{f}\left(x_{1}, \ldots, x_{l+1}\right)=\left(x_{1}, \ldots, x_{l+1},\left|x_{1}+1\right|^{\alpha}\right)$, где $k<\alpha<k+1$, есть цилиндрическое изометрическое погружение класса $C^{k}$, но не $C^{k+1}$. Композиция стандартного вложения единичной сферы в $E^{l+1}$ с центром в начале координат есть $C^{2 k}$-изометрическое вложение, которое единственным образом продолжается до изометрического погружения $D^{l+1}$ в $E^{l+2}$, которое не является $C^{k+1}$-регулярным в точке $(-1,0, \ldots, 0)$.

Как следствие мы получим, что каждое $C^{\infty}$-изометрическое погружение $S^{l} \rightarrow$ $E^{l+2}$ гомотопно посредством $C^{\infty}$-изометрических погружений стандартному вложению сферы в гиперплоскость $E^{l+1}$.

В частности, отсюда следует, что гладкое изометрическое погружение сферы $S^{l}$ $(l \geqslant 3)$ в $E^{l+2}$ не может быть заузлено.

Для $l \geqslant 4$ без установления дифференцируемости гомотопии это было доказано в [174]. Для конечной дифференцируемости это было доказано в [119].

3.3.3. Изометрические погружения метрик постоянной кривизны минимальными подмногообразиями. Пусть $\phi: M^{n} \rightarrow E^{n}$ есть изометрическое погружение $M$ в евклидово пространство. Тогда вектор средней кривизны удовлетворяет уравнению $\Delta \phi=n H$. Если координатные функции погружения являются собственньми функциями, отвечающими одному и тому же собственному значению $\lambda$, то

$$
H=\lambda \phi / n .
$$

Так как $\langle H, d \phi\rangle=0$, то $\langle\phi, d \phi\rangle=0$ и $|\phi|^{2}$ постоянно, а $\phi$ есть погружение в сферу $S^{N-1}$ радиуса $\sqrt{n / \lambda}$. Более того, это есть минимальное погружение в сферу.

Собственные функции на стандартной сфере являются ограничением однородных гармонических полиномов в $E^{n+1}$ на сферу $S^{n}(1)$. Каждому собственному значению $\lambda_{d}=d(d+n+1)$ отвечает подпространство собственных функций $E_{\lambda_{d}}$ размерности

$$
N_{d}=\frac{(2 d+n-1)(d+n-2)}{d !(n-1) !} .
$$

Для нечетного $d$ эти функции задают стандартные минимальные изометрические погружения $f_{n, d}$ стандартной единичной сферы $S^{n}(1)$ в сферу $S^{N_{d}-1}\left(\sqrt{n / \lambda_{d}}\right)$. Для четного $n$ мы получаем изометрическое вложение вещественного проективного пространства $\mathbb{R} P^{n}$ в $S^{N_{d}-1}\left(\sqrt{n / \lambda_{d}}\right)$.

Теорема 3.3 .5 (Калаби [42]). Пусть $S$ есть двумерная сфера с постоянной гауссовой кривизной $K$, и пусть

$$
f: S^{2} \rightarrow S^{n-1}(r) \subset E^{n} \quad(n=2 m+1 \geqslant 3)
$$

- изометрическое минимальное погружение $S^{2}$ такое, что образ не лежит ни в какой гиперплоскости $E^{n}$ (т.е. полное погружение).

Тогда:

1) гауссова кривизна $K$ принимает только значения

$$
K=\frac{2}{m(m+1) r^{2}}
$$


2) погружение однозначно определяется с точностью до движсеня в сфере $S^{n-1}$, и компонентами радиус-вектора изометрического погружения являются соответственно нормированные сферические гармоники порядка т на сфере $S^{2}$, образующие ортонормированный базис.

Даже локально в сфере $S^{n}$ нет минимальных двумерных поверхностей постоянной отрицательной гауссовой кривизны [39], [95].

Все минимальные двумерные поверхности с гауссовой кривизной $K=0$ в сферическом пространстве найдены в [102]. Минимальные поверхности постоянной гауссовой кривизны в евклидовом пространстве суть только области на вполне геодезических двумерных плоскостях нулевой гауссовой кривизны [39]. Аналогичное утверждение справедливо и для пространства Лобачевского $H^{n}$ : минимальная поверхность постоянной гауссовой кривизны в пространстве Лобачевского кривизны - 1 есть только область на вполне геодезической плоскости Лобачевского $H^{2} \subset H^{n}$ кривизны - 1 [103].

М. До Кармо и Н.Р. Валлах [61] показали, что при $d \leqslant 3$ любое полное сферически минимальное изометрическое погружение сферы постоянной секционной кривизны $f: S^{m} \rightarrow S^{n}$ является стандартньм. А также было показано, что при $n>2$ есть много других сферически минимальных изометрических погружений $S^{n}(1)$ в $S^{N}(r)$, отличных от стандартных. Они параметризуются выпукльм телом в конечномерном линейном пространстве. Точные значения размерностей этих вьпуклых тел были найдены в [166], новые конструкции минимальных сферических погружений в [167].

В [61] был поднят вопрос о наименьшей размерности $N$, при которой существует минимальное изометрическое погружение $S^{n}(1)$ в $S^{N}(r)$, которое не является вполне геодезическим. Нижняя граница была дана Дж. Муром в [117]. В [61] М. До Кармо и Н.Р. Валлах предположили, что $N=\frac{n(n+3)}{2}-1$ и это есть вложение Веронезе стандартное вложение, отвечаюшее случаю, когда $d=2$. Но это не так, по крайней мере при $n=3$. Н. Эйжири показал, что существует минимальное изометрическое погружение $S^{3}(1)$ в $S^{6}\left(\frac{1}{4}\right)$, которое не является вполне геодезическим [63], [68].

В [168] доказано, что не существует изометрического минимального погружения сферы $S^{m}$ кривизны $\frac{m}{4 m+3}$ в стандартную единичную сферу $S^{n}$ для $n<\frac{(m+2)(m+7)}{12}-1$.

Для однородных сферических форм имеет место

Теорема 3.3.6 (Детурк, Циллер [59]). Каждая однородная сферическая форма допускает сферически минимальное изометрическое вложсение в стандартную сферу (достаточно высокой размерности и соответствующего радиуса).

Заметим, что однородная сферическая форма принадлежит к одному из следующих классов:

1) $M=S^{3} / \Gamma$, где $Г$ есть конечная подгруппа $S^{3}=S U(r)=S_{p}(1)$;

2) $M=S^{4 n-1} / \Gamma$, где Г есть конечная подгрупша $S_{p}(1)$, действующая на $E^{4 n}=H^{n}$ умножением каждой кватернионной координаты слева;

3) $M=S^{2 n-1} / \mathbb{C}_{d}$, где $\mathbb{C}_{d}$ порождено $e^{2 \pi i / d}$ и действует на $E^{2 n}=\mathbb{C}^{n}$ умножением каждой комплексной координаты.

Минимальные сферические погружения неоднородных сферических форм были рассмотрены в [67]. 
3.3.4. Изометрические погружения проективных пространств в евклидовы пространства. Для изометрического погружения вещественных проективных пространств со стандартной метрикой постоянной секционной кривизны, прежде всего, существуют топологические препятствия. Напомним, что погружение дифференцируемого многообразия $M^{l}$ в дифференцируемое многообразие $V^{l+p}$ есть отображение $f$ класса регулярности $C^{1}$ с якобианом, отличньм от нуля. Если оно представляет собой диффеоморфизм на подмногообразии, то говорят о вложении.

Уитни доказал, что любое многообразие $M^{l}$ может быть погружено в $E^{2 l-1}$ и вложено в $E^{2 l}$. Если мы ограничимся вешественньми проективньми пространствами $\mathbb{R} P^{l}$, то $\mathbb{R} P^{l}$ можно погрузить в $E^{l+p}$ тогда и только тогда, когда над $\mathbb{R} P^{l}$ сушествует поле ортонормированных $l$-реперов, касательных к $\mathbb{R} P^{l+p}[95]$.

Определим $\alpha(l)$ как число единиц в двоичном разложении $l$. Если $l$ есть степень двойки, то $\alpha(l)=1$.

Teоpema 3.3.7 ([95]). а) Пусть $l=2 k+1 u l>1$. Тогда $\mathbb{R} P^{l}$ вкладыввается в $E^{2 l-1}$.

б) Eсли l> 1, то $\mathbb{R} P^{l}$ погружается в $E^{2 l-1}, \mathbb{R} P^{3}-$ в $E^{4}$.

Пусть $l>3 . \quad E c л и l=1 \bmod 2$, то $\mathbb{R} P^{l}$ погружается в $E^{2 l-3}$. Eсли $l=3$ $\bmod 4$, mo $\mathbb{R} P^{l}$ погружается в $E^{2 l-5}$.

Teopema 3.3.8 ([95]).

а) Пусть $l=2 r-1$, где $r \geqslant 4$. Тогда $\mathbb{R} P^{l}$ не погружается в $E^{2 n-q}$, где

$$
\begin{array}{ll}
q=2 r & (r=1,2 \bmod 4), \\
q=2 r+1 \quad & (r=0 \bmod 4), \\
q=2 r+2 & (r=3 \bmod 4) .
\end{array}
$$

б) $\mathbb{R} P^{l}$ погружается в $E^{2 l-q+1}$.

Tеорема 3.3.9 ([95]). Eсли l нечетно и $\alpha(l)>3$, mо $\mathbb{R} P^{l}$ погружается в $E^{2 l-8}$. Eсли $l=3 \bmod 4$ и $\alpha(l)>5$, mо $\mathbb{R} P^{l}$ погружается в $E^{2 l-9}$.

Другие результаты в этом направлении имеются в [95], [109].

Если мы рассматриваем проективное пространство со стандартной метрикой, то добавляются метрические ограничения. В частности, справедлива

Tеорема 3.3.10 (Громов, Рохлин [80]). Если $l$ - степень двойки и $l>1$, то l-мерное вещественное проективное пространство, снабженное римановой метрикой со всюду положительной скалярной кривизной, в частности, $l$-мерное әллиптическое пространство, не допускает изометрического $C^{2}$-вложения ${ }_{\theta} E^{2 l}$.

Доказательство несколько сложнее, чем для двумерного случая. Вместо теоремы Уитни применяется ее обобщение, принадлежащее Маховальду: при указанных значениях $l$ вещественное проективное пространство размерности $l$ нельзя дифференщиально вложить в $E^{2 l}$ с нормальным векторным полем без особенностей. В силу этой теоремы нам достаточно доказать, что если $V$ - риманово многообразие произвольной размерности $l \geqslant 2$ с всюду положительной скалярной кривизной, то при любом 
изометрическом $C^{2}$-погружении $f: V \rightarrow E^{q}$ с $q>l$ на $V$ сушествует нормальное векторное поле без особенностей.

Такое поле строится при любых $l \geqslant 2, q>l$ так же, как вьше оно было построено для $l=2, q=4$, как поле, дуальное следу $\sigma$ второй квадратичной формы. Как и вьше, доказательства требует только тот факт, что этот след, рассматриваемьй как линейная форма на нормальной плоскости $N_{v}$, не обращается тождественно в нуль. Пусть $e_{1}, \ldots, e_{l}$ и $\xi_{1}, \ldots, \xi_{l}$ - ортонормированные базисы в касательной плоскости $T_{v}$ и нормальной плоскости $N_{v}$ соответственно, и пусть $\left(a_{i j}^{k}\right)_{i, j=1}^{n}$ - матрицы квадратичной формы относительно базиса $e_{1}, \ldots, e_{l}$. Согласно формуле Гаусса кривизна многообразия $V$ в точке $v$ по двумерному направлению, определяемому векторами $e_{i}, e_{j}$, равна

$$
\sum_{k}\left[a_{i i}^{k} a_{j j}^{k}-\left(a_{i j}^{k}\right)^{2}\right]
$$

так что скалярная кривизна в этой точке определяется формулой

$$
R=\sum_{i, j, k}\left[a_{i i}^{k} a_{j j}^{k}-\left(a_{i j}^{k}\right)^{2}\right]
$$

Так как $R>0$, то

$$
\sum_{k}\left[\sigma\left(\xi_{k}\right)\right]^{2}=\sum_{k}\left(\sum_{i} a_{i i}^{k}\right)^{2}=\sum_{i, j, k} a_{i i}^{k} a_{j j}^{k} \geqslant R>0
$$

и, следовательно, $\sigma\left(\xi_{k}\right) \neq 0$ при некотором $k$.

Д. Блануша $[25],[19],[20]$ показал, что вешественное проективное пространство $\mathbb{R} P^{l}$ со стандартной метрикой изометрически вкладывается в $E^{N}(N=l(l+3) / 2)$. Если мы возьмем в евклидовом пространстве ортогональные координаты, то сфера $S^{l}$ задается уравнением

$$
\sum_{i=1}^{l+1}\left(x_{i}\right)^{2}=r^{2}
$$

Тогда изометрическое вложение $\mathbb{R} P^{l}$ в $E^{N}(N=n(n+3) / 2)$ задается в виде

$$
x_{i k}=\frac{1}{r \sqrt{2}} x_{i} x_{k} \quad(i=1, \ldots, l+1),
$$

где $x_{i k}$ - ортогональные координаты в $E^{N}$. Это есть многомерное обобшение поверхности Веронезе. Это подмногообразие является стандартным минимальным изометрическим вложением сферы, отвечаюшим второму собственному значению $\lambda_{2}$. Напомним, что четным собственньм значениям отвечают минимальные изометрические вложения проективных пространств в сферы. При этом все движения $\mathbb{R} P^{l}$ индуцируются движениями объемлющего пространства. При этом вложении геодезические линии являются окружностями евклидова пространства. Если к изометрии добавить это дополнительное условие, то указанная оценка размерности объемлющего 
пространства неулучшаема и вложение единственно с точностью до движения объемлюшего пространства. Это вложение является свободным, т.е. в каждой точке векторы $\frac{\partial r}{\partial u_{i}}, \frac{\partial^{2} r}{\partial u_{i} \partial u_{j}}$ линейно независимы, где $r=r(u), u=\left(u_{1}, \ldots, u_{l}\right)$ - локальная параметризация подмногообразия. То есть размерность второго соприкасающегося пространства равна $(l+3) l / 2$. В $[25]$ построены другие примеры изометрических вложений $\mathbb{R} P^{l}$ в евклидовы пространства. Они основаны на обобщении вложения (3.3.4). И в этом случае все движения $\mathbb{R} P^{l}$ индуцируются движениями объемлющего пространства.

Линзовое пространство $L^{n}\left(r^{m}\right)$ есть фактор-пространство сферы $S^{2 n+1}$ при свободном действии циклической групшы $Z / r^{m}$, заданном в виде

$$
\xi^{k} z=\left\{\xi^{k} z_{0}, \ldots, \xi^{k} z_{n}\right\}
$$

где $\xi=\exp \left(i \pi / r^{m-1}\right)$ есть образуюшая групшы $Z / r^{m}$.

ТЕОРема 3.3.11 (Бернард [16]). При $m \geqslant\left[\log _{2} n\right]+[n / 2]$ линзовое пространство $L^{n}\left(r^{m}\right)$ не допускает изометрического погружсения в $E^{4 n-\alpha(n)}$, где $\alpha(n)$ - число единии в двоичном разложсении п.

Глава 4. Изометрические погружения псевдоримановых пространств постоянной кривизны

Пусть $E_{s}^{l}$ - вещественное векторное пространство со скалярньм произведением сигнатуры $(s, l-s+1)$, заданным в виде

$$
\langle X, Y\rangle=-\sum_{i=1}^{s} x_{i} y_{i}+\sum_{j=s+1}^{l+1} x_{j} y_{j}
$$

для векторов $X=\left(x_{1}, \ldots, x_{l+1}\right), Y=\left(y_{1}, \ldots, y_{l+1}\right), c=$ const $>0$.

Гиперповерхность $S_{s}^{l}(c)$,

$$
\langle X, X\rangle=\frac{1}{c^{2}}
$$

есть псевдориманово пространство постоянной положительной кривизны $c$ сигнатуры $(s, l-s)$.

Гиперповерхность $H_{s}^{l}(c)$,

$$
\langle X, X\rangle=-\frac{1}{c^{2}},
$$

есть псевдориманово пространство сигнатуры $(s-1, l-s+1)$ постоянной отрицательной кривизны $-c$.

При $s=0 S_{0}^{l}(c)=S^{l}(c)$ есть стандартная сфера в евклидовом пространстве, при $s=0 H_{0}^{l}(c)$ есть пространство Лобачевского $H^{l}$. При изменении знака в скалярном произведении (4.0.1) в $E_{s}^{l+1}$ мы получим скалярное произведение в $E_{l+1-s}^{l+1}$ и $S_{s}^{l}(c)$ перейдет в $H_{l-s}^{l}(-c)$. Поэтому все результаты относительно сфер $S_{s}^{l}(c)$ переходят в утверждение относительно гиперболических пространств $H_{l-s}^{l}(-c)$. Каждое вполне геодезическое $m$-мерное подмногообразие $S_{s}^{l}\left(H_{s}^{l}\right)$ есть связная компонента пересечения $S_{s}^{l}\left(H_{s}^{l}\right)$ с $(m+1)$-мерньм линейным подпространством $E_{s}^{l+1}\left(E_{s+1}^{n+1}\right)$. Сфера $S_{s}^{l}$ 
диффеоморфна $S^{l-s} \times E^{s}, H_{s}^{l}$ диффеоморфно $S^{s} \times E^{l-s}$. В псевдоевклидовом пространстве $E_{1}^{3}$ сфрера $S_{1}^{2}$ с метрикой сигнатуры $(1,1)$ имеет положительную кривизну и является в $E_{1}^{3}$ однополостным гиперболоидом врашения. $H_{0}^{2}=H^{2}$ есть двухполостньй гиперболоид и одна полость изометрична пространству Лобачевского. Но в $E_{1}^{3}$ не существует поверхности класса $C^{1}$ с индуцированной полной римановой метрикой постоянной положительной гауссовой кривизны. Более того, замкнутое двумерное многообразие с метрикой постоянной сигнатуры не допускает $C^{1}$-изометрического погружения в $E_{1}^{3}$ [158].

В $E_{1}^{3}$ не существует регулярной внешне полной поверхности с индефинитной метрикой и постоянной отрицательной кривизной, предельный конус которой всюду гладкий, кроме вершины [157].

Естественно возникает вопрос о единственности с точностью до движения в $E_{1}^{3}$ изометрического погружения плоскости Лобачевского в $E_{1}^{3}$. Оказалось, что для единственности с точностью до параллельного переноса и отражения нужно, кроме метрики и предельной образующей, фиксировать еще некоторую линейчатую поверхность, которая апшроксимирует данную поверхность на бесконечности более точно, чем предельный конус. Тогда существует единственная внешне полная вьпуклая поверхность с метрикой постоянной отрицательной кривизны с изотропньм предельньм конусом с заданной аппроксимацией линейчатой поверхностью на бесконечности [129].

ЗАмЕЧАНИЕ. Из внешней полноты не следует полнота метрики поверхности. Поэтому, как мне кажется, вопрос единственности изометрического погружения полной плоскости Лобачевского без асимптотики на бесконечности остается открытьм.

4.1. Погружения нулевой внешней кривизны. Локальное и глобальное строение изометрических погружений псевдоевклидова (лоренцева) пространства $E_{1}^{l}$ в $E_{1}^{l+1}$ изучал Л. Грейвс [76]. Оценка для внешнего нуль-индекса в этом случае та же, что и в евклидовом, $\mu \geqslant l-1$. Справедлив аналог леммы 3.1.1. Только под полнотой слоев мы понимаем геодезическую полноту.

Вектор $X$ в $E_{1}^{l+1}$, для которого $\langle X, X\rangle=1$, назьвается пространственноподобным, если же $\langle X, X\rangle=-1$, то вектор назьвается времениподобным. Если $\langle X, X\rangle=0$, то вектор называется светоподобньм или нуль-вектором. Нуль-репер в $E_{1}^{3}$ - это тройка векторов

$$
F=(A, B, C)=\left(\begin{array}{lll}
a_{0} & b_{0} & c_{0} \\
a_{1} & b_{1} & c_{1} \\
a_{2} & b_{2} & c_{2}
\end{array}\right),
$$

где $A$ и $B$ - нуль-векторы, для которых $\langle A, B\rangle=-1, C$ - единичный пространственноподобньй вектор, ортогональньй плоскости, натянутой на векторы $A$ и $B$, и $\operatorname{det} F=+1$. Регулярная кривая $s \rightarrow F(s)$ в связной подгруппе движений $E_{1}^{3}$, содержащей тождественное преобразование $S O^{+}(2,1)$, называется реперной кривой. Для реперной кривой справедливы уравнения Френе:

$$
\begin{aligned}
& \frac{d A}{d s}=k_{1}(s) A(s)+k_{2}(s) C(s), \\
& \frac{d B}{d s}=-k_{1}(s) B(s)+k_{3}(s) C(s), \\
& \frac{d C}{d s}=k_{3}(s) A(s)+k_{2}(s) B(s) .
\end{aligned}
$$


Кривая $x(s)$ в $E_{1}^{3}$ назьвается нуль-кривой, если в каждой точке $\left\langle\frac{d x}{d s}, \frac{d x}{d s}\right\rangle=0$, где $\langle\cdot, \cdot\rangle$ - скалярное произведение в $E_{1}^{3}$.

Если $x(0)=0$, то нуль-кривая лежит на изотропном конусе. Для нуль-кривой можно выбрать нуль-репер так, что $\frac{d x}{d s}$ пропорционально $A(s)$. Можно выбрать вектор $C(s)$, аналогичный главной нормали кривой в евклидовом случае, так, что уравнения Френе имеют вид:

$$
\frac{d x}{d s}=A(s), \quad \frac{d A}{d s}=k_{2}(s) C(s), \quad \frac{d B}{d s}=k_{3}(s) C(s), \quad \frac{d C}{d s}=k_{3}(s) A(s)+k_{2}(s) B(s) .
$$

Этот репер назьвается репером Картана для нуль-кривой. Рассмотрим линейчатую ( $B$-линейчатую) поверхность с параметризацией

$$
f(s, u)=x(s)+u B(s) .
$$

Она несет индуцированную индефинитную (лоренщеву) метрику. Она изометрична лоренцевой плоскости $E_{1}^{2}$, только когда $k_{3}(s)=0$, т.е. когда $\frac{d B}{d s}=0$ и $B(s)$ есть параллельное изотропное векторное поле. В этом случае $B$-линейчатую поверхность будем называть изотропньм цилиндром. Заметим, что направляющая этого цилиндра, в отличие от евклидова случая, не лежит в двумерной плоскости. Она лежит на изотропном конусе.

Если изометрическое регулярное погружение $f: E_{1}^{l} \rightarrow E_{1}^{l+1}$ не является вполне геодезическим, то найдется точка $P$ с внешним нуль-индексом $\mu=l-1$ и нуль-индекс будет постоянньм в окрестности этой точки. Из аналога леммы 3.1 .1 следует, что на образе $F_{1}^{l}=f\left(E_{1}^{l}\right)$ лежит полное линейчатое подпространство $T_{l-1}(P)$ объемлюшего пространства. При этом возможны следуюшие случаи:

1) $T^{l-1}(P)$ есть лоренщево подпространство $E_{1}^{l-1}$;

2) $T^{l-1}(P)$ есть евклидово подпространство $E^{l-1}$;

3) $T^{l-1}=E^{l-2} \oplus \operatorname{Span}\{\xi\}$, где $E^{l-2}$ - евклидово пространство, $\xi$ - нуль-вектор и $\langle y, \xi\rangle=0$ для всех $y \in E^{l-2}$.

В случаях 1), 2) метрика $T^{l-1}(P)$ не вырождена, в случае 3$)$ вырождена.

Имеет место аналог теоремы Хартмана-Ниренберга [82].

Теорема 4.1 .1 (Грейвс [76]). Регулярное изометрическое погружсение $f: E_{1}^{l} \rightarrow$ $E_{1}^{l+1}$ имеет один из следующих видов.

1) $E_{1}^{l}=E_{1}^{l-1} \times E^{1} \stackrel{\text { id } \times c}{\longrightarrow} E_{1}^{l-1} \times E_{2}$, әде c- плоская кривая в евклидовой плоскости. Это - чилиндр с образующей $E_{1}^{l-1}$ над плоской евклидовой кривой.

2) $E_{1}^{l}=E^{l-1} \times E_{1}^{1} \stackrel{\text { id } \times c}{\longrightarrow} E^{l-1} \times E_{1}^{2}$, где c- плоская времениподобная кривая в псевдоевклидовой плоскости. Это - иилиндр с евклидовой образующей $E^{l-1}$ над плоской времениподобной кривой.

3) $E_{1}^{l}=E^{l-2} \times E_{1}^{2} \stackrel{\text { id } \times g}{\longrightarrow} E^{l-2} \times E_{1}^{3}$, где $g: E_{1}^{2} \rightarrow E_{1}^{3}$ есть изотропньй иилиндр. Это - чилиндр с евклидовой образующей $E^{l-2}$ над изотропным иилиндром в $E_{1}^{3}$.

Все произведения являются ортогональными в метрике объемлющего пространства. Гиперповерхность $f\left(E_{1}^{l}\right) \subset E_{1}^{l+1}$, изометричная $E_{1}^{l}$, является цилиндром с $(l-1)$-мерной образующей. Образуюшими этого цилиндра могут быть

1) лоренцево подпространство $E_{1}^{l-1}$, 
2) евклидово подпространство $E^{l+1}$,

3) подпространство $E^{l-2} \oplus \operatorname{Span}\{\xi\}$ с вырожденной метрикой.

Случай изометрического вложения $E_{s}^{l}$ в $E_{s+k}^{l+p}$ с внешним нуль-индексом $\mu \geqslant l-1$ был рассмотрен в [4], получены результаты, аналогичные теореме 4.1.1. Однако без внешнего ограничения на нуль-индекс вопрос о строении подмногообразия $F^{l} \subset E_{s}^{l+p}$, изометричного $E_{s}^{l}$, остается открытым.

Из статей [36], [38] следует

ТеОРема 4.1.2. Пусть $f: E_{s}^{l} \rightarrow E_{s}^{l+p}$ есть изометрическое погружсение $c$ постоянным внешним нуль-индексом. Если в некоторой точке $Q_{0}$ нуль-пространство $L^{k}\left(Q_{0}\right)$ есть псевдоевклидова плоскость $E_{s}^{k}$, то подмногообразие $f\left(E_{s}^{l}\right)$ есть иилиндр с образующей $E_{s}^{l-p}$.

Для псевдоевклидова пространства $E_{1}^{n}$ справедлива лемма, аналогичная лемме 2.1.1.

Лемма 4.1.1 ([36], [38]). Предположимм, что $M_{1}^{2}$ есть поверхность класса $C^{1}$ в псевдоевклидовом пространстве $E_{1}^{n}$, изометричная полосе на лоренцевой плоскости $E_{1}^{2}$ между параллельными времениподобнымми прямымми. Если существует времениподобная прямая обгемлющего пространства, которая лежит на поверхности $M_{1}^{2}$, то поверхность есть цилиндр с времениподобной образующей.

ДокАЗАТЕльство. Пусть $\bar{l}$ - прямая на поверхности $M_{1}^{2}$, и пусть $l$ - соответствующая прямая на $E_{1}^{2}, \bar{l}=\phi(l)$, где $\phi$ - изометрия между $E_{1}^{2}$ и $M_{1}^{2}$. Параметризуем $l$ как $\gamma(t), t \in \mathbb{R}$, и для каждого $t \lambda_{t}$ есть сегмент, ортогональньй к $l$ в точке $\gamma(t)$. Тогда $\phi\left(\lambda_{t}\right)=\bar{\lambda}_{t}$ лежит в подпространстве $E_{1}^{n}$, ортогональном к $\bar{l}$ в псевдоевклидовой метрике $E_{1}^{n}$. Допустим, что это не так в точке $\gamma\left(t_{0}\right)=q_{0}$, не ограничивая обшности, можно считать, что $t_{0}=0$. По предположению существует точка $p \in E_{1}^{2}$ такая, что сегмент $\bar{q}_{0} \bar{p}$ в $E_{1}^{n}$ не является ортогональньг к $\bar{l}$ в объемлющем пространстве, где $\bar{q}_{0}=\phi\left(q_{0}\right)$ и $\bar{p}=\phi(p)$. Обозначим длину сегмента $q_{0} p_{0}$ через $h$ и $\bar{\gamma}(t)=\phi(\gamma(t))$. Предположим, что $t$ настолько велико, что стороны треугольника $\gamma(-t) p \gamma(t)$ являются времениподобными. Если $t$ есть длина прямой $l$ от точки $q_{0}=\gamma(0)$ до точки $\gamma(t)$, TO

$$
|p \gamma(t)|^{2}=|p \gamma(-t)|^{2}=\left|-t^{2}+h^{2}\right|=t^{2}-h^{2} .
$$

Выберем в $E_{1}^{n}$ ортогональную систему координат так, что прямая $\bar{l}$ совпадает с осью $x_{0}$, точка $\bar{q}_{0}$ - начало координат, точка $\bar{p}$ принадлежит плоскости $x_{0}, x_{1}$. Тогда координаты точки $\bar{p}$ суть $\left(t_{0}, \bar{h}, 0, \ldots, 0\right)$ с $t_{0}>0$ (или $\left.<0\right)$ и

$$
\begin{aligned}
|\bar{p} \bar{\gamma}(t)|^{2} & =\left|-\left(t-t_{0}\right)^{2}+\bar{h}^{2}\right|=\left(t-t_{0}\right)^{2}-\bar{h}^{2}, \\
|\bar{p} \bar{\gamma}(-t)|^{2} & =\left|-\left(t+t_{0}\right)^{2}+\bar{h}^{2}\right|=\left(t+t_{0}\right)^{2}-\bar{h}^{2} .
\end{aligned}
$$

Так как $\phi$ есть изометрия, то $t^{2}-h^{2}=d(\bar{p}, \bar{\gamma}(t))^{2}$, где $d$ - расстояние на $M_{1}^{2}$. Используя тот факт, что времениподобная прямая имеет наибольшую длину среди всех времениподобных кривых, соединяющих две фиксированные точки, мы получим, что

$$
\begin{aligned}
& \left(t-t_{0}\right)^{2}-\bar{h}^{2} \geqslant t^{2}-h^{2}, \\
& \left(t+t_{0}\right)^{2}-\bar{h}^{2} \geqslant t^{2}-h^{2} .
\end{aligned}
$$


Отсюда следует, что

$$
\begin{aligned}
-2 t t_{0}+t_{0}^{2} & \geqslant \bar{h}^{2}-h^{2}, \\
2 t t_{0}+t_{0}^{2} & \geqslant \bar{h}^{2}-h^{2},
\end{aligned}
$$

что невозможно при большом $t$. И мы пришли к противоречию.

Рассмотрим четырехугольник $q_{0} \gamma(t) p(t) p_{0}$ на плоскости $E_{1}^{2}$, где $q_{0} \in l$ и сегменты $g_{0} p_{0}$ и $\gamma(t) p(t)$ ортогональны к $l$. Соответствуюший четырехугольник в $E_{1}^{n}$ есть $\bar{q}_{0} \bar{\gamma}(t) \bar{p}(t) \bar{p}_{0}$. Мы возьмем в $E_{1}^{n}$ предыдушую систему координат, в ней $\bar{p}_{0}=\left(0, \bar{h}_{0}, 0, \ldots, 0\right), \bar{p}(t)=(t, \bar{h}, \cos \alpha, \bar{h}, \sin \alpha, 0, \ldots, 0)$, где $\alpha-$ угол между $\bar{g}_{0} \bar{p}_{0}$ и $\bar{\gamma}(t) \bar{p}(t)$.

Мы знаем, что

$$
\left|\bar{p}_{0} \bar{p}(t)\right|^{2}=t^{2}-\left(\bar{h}_{0}-\bar{h}_{1} \cos \alpha\right)^{2}-\left(\bar{h}_{1} \sin \alpha\right)^{2} .
$$

Так как $\phi-$ изометрия, то

$$
\left|\bar{p}_{0} \bar{p}(t)\right|^{2} \geqslant\left|p_{0} p(t)\right|^{2}=t^{2}
$$

Тогда $t^{2}-\left(\bar{h}_{0}-\bar{h}_{1} \cos \alpha\right)^{2}-\left(\bar{h}_{1} \sin \alpha\right)^{2} \geqslant t^{2}, \bar{h}_{0}=\bar{h}_{1}, \alpha=0$ и $\left|\bar{p}_{0} \bar{p}(t)\right|=t$. Это значит, что отрезок $\bar{p}_{0} \bar{p}(t)$ лежит на поверхности $M_{1}^{2}$ и параллелен прямой $\bar{l}$.

4.2. Изометрические погружения псевдоримановых пространств ненулевой постоянной кривизны. Изометрические погружения псевдоримановых пространств постоянной положительной кривизны единица $S_{s}^{l}(1)$ в $S_{s+t}^{l+p}(1)$ рассмотрены в работах [52]-[54], [77]. Найдены условия вполне геодезичности изометрического погружения. Имеет место результат, аналогичный теореме 3.1.2 для стандартного сферического пространства.

Теорема 4.2.1 (Дайчер, Форнари [53]). Пусть $f: S_{s}^{l}(1) \rightarrow S_{s}^{l+p}(1)$ есть регулярное изометрическое погружение. Если $p<l-s$, то $f\left(S_{s}^{l}(1)\right)$ есть вполне геодезическое подмногообразие.

Для $s=1, l=2, p=1$ в [77] построен пример изометрического погружения $S_{1}^{2} \rightarrow S_{1}^{3}$, которое не является вполне геодезическим.

При дополнительном условии на нуль-пространства как следствие из более общей теоремы справедлива

ТЕОРема 4.2 .2 (Эйб, Мейгид [3]). Пусть $f: S_{s}^{l}(c) \rightarrow S_{s}^{l+p}(c)$ - изометрическое погружение псевдоримановых пространственных форм. Пусть для точки с минимальным нуль-индексом нуль-пространство $L^{k}$ есть псевдоевклидова плоскость $E_{s}^{k}$. Eсли $p<l$, то подмногообразие $F_{s}^{l}=f\left(S_{s}^{l}(c)\right)$ есть вполне геодезическое подмногообразие в $S_{s}^{l+p}(c)$.

Аналогичная теорема для изометрического погружения $f: S^{l}(c) \rightarrow S_{p}^{l+p}(c)$ следует из более ранней работы [33]. Если $p<l$, то $F^{l}=f\left(S^{l}\right)$ является вполне геодезическим стандартным подмногообразием. 
Теорема 4.2.3 (Дайчер, Домбровский [52]). Пусть $f: S_{s}^{l}(1) \rightarrow S_{s+1}^{l+p}(1)$ ecmь peгулярное изометрическое погружение с невырожденным нуль-пространством минимальной размерности. Если $p<\frac{1}{2}(l-s)$, то $f\left(S_{s}^{l}(1)\right)$ есть вполне геодезическое подмногообразие $S_{s+1}^{l+p}$.

Без дополнительных условий теорема не верна. Существуют изометрические погружения $f: S_{s}^{l}(1) \rightarrow S_{s+1}^{l+2}$ при $0 \leqslant s \leqslant l-1$, которые не являются вполне геодезическими подмногообразиями [53]. Описание таких погружений дано в [54].

Дело в том, что если сигнатуры метрик вложенного подмногообразия и объемлющего пространства не совпадают, то нормальное расслоение не риманово, а лоренцево, и образ второй квадратичной формы может содержаться в нуль-направлении, и тогда мы не имеем оценки снизу на внешний нуль-индекс.

Полное описание изометрических погружений псевдориманова пространства $H_{1}^{l}$ постоянной отрицательной кривизны -1 в пространство $H_{1}^{l+1}$ той же кривизны дано в [1] на языке геодезических ламинаций. Это описание аналогично описанию изометрических погружений пространства Лобачевского $H^{l}(-1)$ в $H^{l+1}(-1)$ (теорема 3.1.6).

Локальные и глобальные изометрические погружения псевдориманова пространства постоянной отрицательной кривизны $H_{s}^{l}$ в псевдоевклидово пространство $E_{s}^{n}$ изучались в [32].

ТеОрема $4.2 .4([32])$. Пусть $H_{s}^{l}$ - псевдориманово пространство постоянной отрицательной кривизны, $E_{s}^{n}-$ псевдоевклидово пространство. Eсли $n<2 l-1$, то многообразие $H_{s}^{l}$ локально изометрично не погружсатся в $E_{s}^{n} ;$ если $n=2 l-$ 1, то локально изометрическое погружение существует.

Когда $s=0$, получается следуюшая известная теорема: $l$-мерное пространство Лобачевского нельзя локально изометрично вложить в евклидово пространство $E^{2 l-2}$, и сушествует локально изометрическое вложение в $E^{2 l-1}$. Эту теорему открыл Э. Картан в 1919 году, в 1938 году ее переоткрыл А.Е. Либер; пример локального вложения построил $\Phi$. Шур в 1886 году. На вопрос о глобальном погружении полного псевдориманова пространства постоянной отрицательной кривизны частично отвечает

ТеОРема 4.2 .5 [32]. Пусть $H_{s}^{l}$ - полное связное псевдориманово многообразие постоянной отрицательной кривизны. Если $s \neq 0,1,3,7$, то многообразие $H_{s}^{l}$ не погружается изометрично в $E_{s}^{2 l-1}$.

Есть основания считать, что при $s=0,1,3,7$ теорема 4.2.5 также справедлива, но доказательство ее в этом случае автору не известно. Теоремы 4.2.4, 4.2.5 обобщаются естественным образом на случай, когда объемлюшим пространством является псевдориманово многообразие постоянной кривизны. Справедлива

ТЕОРема 4.2.6 [32]. Пусть $H_{s}^{l}$ - полное связное псевдориманово многообразие постоянной кривизны $c, H_{s}^{2 l-1}$ - полное связное псевдориманово многообразие постоянной кривизны $C$, причем $c<C$. Если многообразие $H_{s}^{l}$ является регулярной поверхностью в $H_{s}^{2 l-1}$ u $s \neq 0,1,3,7$, то $C>0, c=0$ и многообразие $H_{s}^{l}$ локально изометрично псевдоевклидову пространству.

Заметим, что в римановом пространстве имеет место родственная теорема 3.2.9. Для псевдоримановых пространств справедлива 
ТЕОРема 4.2.7 (Вольф [175]). Пусть $H_{s}^{l}$ - полное связное псевдориманово многообразие постоянной кривизны $K$. Универсальной накрывающей многообразия $H_{s}^{l}$ является гиперповерхность

а) $\sum_{i=1}^{p+1} x_{i}^{2}-\sum_{j=1}^{s} x_{p+1-j}^{2}=K^{-1 / 2}$ в пространстве $E_{s}^{l+1}$, если $p \neq 1, K>0$;

б) $\sum_{i=1}^{p} x_{i}^{2}-\sum_{j=1}^{s+1} x_{p+j}^{2}=-K^{-1 / 2}$ в пространстве $E_{s}^{l+1}$, если $s \neq 1, K<0$;

и псевдоевклидово пространство $E_{s}^{l}$, если $K=0$.

ДокАЗАТЕЛЬСТво теоРемЫ 4.2.4. Допустим противное: пусть $H_{s}^{l}$ - регулярная $l$-мерная поверхность постоянной отрицательной секционной кривизны в $E_{s}^{2 l-2}$. Не ограничивая обшности, можно считать кривизну по двумерньм площадкам равной -1 . Пусть $Q$ - произвольная точка поверхности $H_{s}^{l} ; T_{Q}$ - касательное пространство в точке $Q ; x=\left(x^{1}, \ldots, x^{l}\right)$ - произвольньй вектор в $T_{Q} ; g=g_{i j} x^{i} x^{j}$ - метрическая форма поверхности в точке $Q$. Ранг квадратичной формы $g$ в каждой точке равен $l, A^{m}=A_{i j}^{m} x^{i} x^{j}$ - вторая квадратичная форма поверхности в точке $Q$, соответствующая нормали $n_{m}(m=1, \ldots, l-2)$. Так как все нормали поверхности являются пространственноподобными векторами, то нормали $n_{m}$ возьмем так, чтобы они были взаимно ортогональными с нормой, равной 1 . По формуле Гаусса тензор кривизны поверхности $H_{s}^{l}$ равен

$$
R_{i j k l}=\sum_{m}\left(A_{i k}^{m} A_{j i}^{m}-A_{i l}^{m} A_{j k}^{m}\right)
$$

Так как поверхность $H_{s}^{l}$ является пространством постоянной отрицательной кривизны, то

$$
R_{i j k l}=-\left(g_{i k} g_{j i}-g_{i l} g_{j k}\right) .
$$

Из формул (4.2.1) и (4.2.2) непосредственно следует

$$
\sum_{m}\left(A_{i k}^{m} A_{j i}^{m}-A_{i l}^{m} A_{j k}^{m}\right)+\left(g_{i k} g_{j i}-g_{i l} g_{j k}\right)=0
$$

Рассмотрим систему $l-1$ уравнений:

$$
\begin{aligned}
A_{i j}^{m} x^{i} x^{j} & =0 \quad(m=1, \ldots, l-2), \\
g_{i j} x^{i} x^{j} & =0 .
\end{aligned}
$$

Из условия (4.2.3) следует, что система (4.2.4) удовлетворяет условию леммы 3.1.1. Значит, система (3.2.1) имеет нетривиальное решение $x_{0}$. Тогда из уравнения (4.2.3) следует

$$
-\sum_{m}\left(A^{m}\left(x_{0}, y\right)\right)^{2}-g^{2}\left(x_{0}, y\right)=0
$$

для любого $y \in T_{Q}$.

Из (4.2.5) следует, что $g\left(x_{0}, y\right)=0$ для любого $y \in T_{Q}$. Это значит, что квадратичная форма $g$ имеет ранг меньше $l$. Мы пришли к противоречию. 
Если $n=2 l-1$, то локальное изометрическое вложение многообразия $H_{s}^{l}$ постоянной отрицательной кривизны в пространство $E_{s}^{2 l-1}$ задается следующим образом:

$$
\begin{array}{r}
y_{2 i-1}= \begin{cases}a_{i} e_{e}^{u} \cos \frac{u_{i}}{a_{i}}, & 1 \leqslant i \leqslant p-1 ; \\
a_{i} e_{e}^{u} \operatorname{ch} \frac{u_{i}}{a_{i}}, & p \leqslant i \leqslant l-1 ;\end{cases} \\
y_{2 i}= \begin{cases}a_{i} e_{e}^{u} \sin \frac{u_{i}}{a_{i}}, & 1 \leqslant i \leqslant p-1 ; \\
a_{i} e_{e}^{u} \operatorname{sh} \frac{u_{i}}{a_{i}}, & p \leqslant i \leqslant l-1 ;\end{cases}
\end{array}
$$

$y_{2 l-1}=\int_{0}^{u_{e}} \sqrt{1-e^{2 u}}$, где $y_{1}, \ldots, y_{2 l-1}-$ координаты в $E_{s}^{2 l-1}, a_{1}, \ldots, a_{l}$ - вешественные числа, удовлетворяюшие условию $\sum_{i} a_{i}^{2}=1$.

При доказательстве теоремы 4.2.5 достаточно ограничиться случаем, когда $H_{s}^{l}$ - полное односвязное псевдориманово многообразие постоянной отрицательной кривизны. Действительно, если в пространство $E_{s}^{2 l-1}$ изометрично погружается полное многообразие $H_{s}^{l}$, то изометрично погружается в $E_{s}^{2 l-1}$ универсальное накрывающее многообразие.

ДокАЗАТЕЛЬСтво теоремы 4.2.5. Допустим противное: пусть $H_{s}^{l}$ - полная односвязная поверхность постоянной отрицательной кривизны в $E_{s}^{2 l-1}$. Из уравнений (4.2.4) следует, что в каждой точке поверхности симметричные билинейные формы $A^{m}=A_{i j}^{m} x^{i} y^{j}, g=g_{i j} x^{i} y^{j}(m=1, \ldots, l-1)$ внешне ортогональны. Так как ранг формы $g$ равен $l$, то билинейные формы $A^{m}, g$ удовлетворяют условию теоремы Картана (теорема 3.2 .3 ). Поэтому в каждой точке $Q$ поверхности $H_{s}^{l}$ в касательном пространстве $T_{Q}$ существует единственньй базис, в котором формы $A^{m}, g$ диагональны. Базис образуют ортогональные векторы $e_{1}, \ldots, e_{p+s}$, так как форма $g$ - диагональна. Заметим, что векторы $e_{1}, \ldots, e_{p}$ пространственноподобны, а векторы $e_{p+1}, \ldots, e_{p+s}$ времениподобны, так как их норма $g_{i j} e_{k}^{i} e_{k}^{j}>0$ при $k \leqslant p$ и $<0$ при $k>p$. По теореме Вольфа многообразие $H_{s}^{l}$ глобально изометрично гиперповерхности $H_{s}^{l}$, заданной уравнением

$$
\sum_{i=1}^{p} x_{i}^{2}-\sum_{j=1}^{s+1} x_{p+j}^{2}=-1
$$

в псевдоевклидовом пространстве $E_{s+1}^{l+1}$. Рассмотрим сфферу $S^{s}$, расположенную на гиперповерхности $H_{s}^{l}$ и заданную уравнениями $x_{i}=0(i=1, \ldots, p)$. Пусть $T^{s} \subset T_{Q}$ - касательное пространство к сфере $S^{s}$ в точке $Q$. Оно состоит из времениподобных векторов. Выберем в касательном пространстве $T_{Q}$ следуюший ортогональный базис: времениподобные векторы $r_{p+1}, \ldots, r_{p+s}$ образуют ортогональный базис в плоскости $T^{s}$, пространственноподобные векторы $r_{1}, \ldots, r_{p}$ расположены в ортогональном дополнении к $T^{s}$. Тогда

$$
l_{p+j}=\sum_{i=1}^{l} \lambda_{p+j}^{i} r_{i} \quad(j=1, \ldots, s) .
$$


Докажем, что

$$
\operatorname{det}\left(\lambda_{p+j}^{p+i}\right) \neq 0 \quad(i, j=1, \ldots, s)
$$

Допустим, что это не так. Тогда существует ненулевой вектор $e=\sum_{j=1}^{s} \mu_{j} e_{p+j}=$ $\sum_{i=1}^{p} \nu_{i} r_{i}$, который является линейной комбинацией, с одной стороны, независимых времениподобных векторов $e_{p+j}$, с другой стороны - независимых пространственноподобных векторов $r_{i}(i=1, \ldots, p)$.

Поэтому вектор $е$ одновременно является пространственноподобным, чего не может быть. Векторы $e_{p+j}(j=1, \ldots, s)$ спроектируем ортогонально в смысле псевдоевклидовой метрики касательного пространства $T_{Q}$ на подпространство $T^{s}$. Из неравенства нулю детерминанта (4.2.6) следует, что векторы $e_{p+j}$ перейдут в линейно независимые векторы $e_{p+j}^{\prime}$, касательные к сфере $S^{s}$. Они параллелизуют сферу $S^{s}$. По теореме Адамса параллелизуемы только сферы $S^{1}, S^{3}, S^{7}$. Мы пришли к противоречию с нашим предположением.

ДоКАЗАТЕЛЬСТВо ТЕОРЕМЫ 4.2.6. Полная односвязная поверхность $H_{s}^{l}$ является поверхностью постоянной отрицательной внешней кривизны в многообразии $H_{s}^{2 l-1}$. По формуле Гаусса тензор кривизны поверхности $H_{s}^{l}$ равен

$$
R_{i j k l}=\sum_{m=1}^{l-1}\left(A_{i k}^{m} A_{j l}^{m}-A_{i l}^{m} A_{j k}^{m}\right)+C\left(g_{i k} g_{j l} \cdot g_{i l} g_{j k}\right)
$$

Из формул (4.2.2) и (4.2.7) следует, что билинейные формы $A^{m}(m=1, \ldots, l-1)$, $g$ являются внешне ортогональными. Единственньй базис, в котором формы диагональны, параллелизует поверхность $H_{s}^{l}$. Дальнейшее доказательство невозможности случая $c<0$ аналогично доказательству теоремы 4.2.5. При доказательстве невозможности случая $c>0$ надо учесть, что поверхность $H_{s}^{l}$ глобально изометрична гиперповерхности

$$
\sum_{i=1}^{p+1} x_{i}^{2}-\sum_{j=1}^{s} x_{p+1+j}^{2}=c^{-1 / 2}
$$

в псевдоевклидовом пространстве $E_{s^{\prime}}^{l+1}$. При доказательстве мы параллелизуем сферу $S^{p}$, заданную уравнениями $x_{p+1+j}=0(j=1, \ldots, s)$. Поэтому получаются ограничения и на $p$.

Пусть $S_{s}^{2 l-1}$ - гиперповерхность в $E_{s}^{2 l}$, заданная уравнением

$$
\sum_{k=1}^{2 p} x_{k}^{2}+\sum_{j=1}^{s} x_{2 p+2 j-1}^{2}-\sum_{j=1}^{s} x_{2 p+2 j}^{2}=1
$$


Пусть $H_{s}^{l}-l$-мерная полная поверхность в многообразии $S_{s}^{2 l-1}$, заданная уравнениями

$$
\begin{gathered}
x_{2 i-1}= \begin{cases}\frac{1}{\sqrt{2 l}} \cos u_{i}, & \text { если } 1 \leqslant i \leqslant p ; \\
\frac{1}{\sqrt{2 l}} \operatorname{ch} u_{i}, & \text { если } p+1 \leqslant i \leqslant l ;\end{cases} \\
x_{2 i}= \begin{cases}\frac{1}{\sqrt{2 l}} \sin u_{i}, & \text { если } 1 \leqslant i \leqslant p ; \\
\frac{1}{\sqrt{2 l}} \operatorname{sh} u_{i}, & \text { если } p+1 \leqslant i \leqslant l .\end{cases}
\end{gathered}
$$

Секционная кривизна поверхности $V_{s}^{l}$ тождественно равна нулю, кривизна гиперповерхности $S_{s}^{2 l-1}$ равна 1. Этот пример реализует случай, которьй допускает теорема 4.2.6.

Аналогичные теоремы справедливы для двойственного пространства постоянной положительной кривизны.

\section{Глава 5. Солитоны и геометрия}

5.1. Солитоны и псевдосферические поверхности. Уравнения изометрических погружений пространственных форм постоянной кривизны в пространства постоянной кривизны имеют те же свойства, что солитонные уравнения, в частности, они имеют пару Лакса и преобразования Беклунда [163].

Вообще, первое солитонное уравнение синус Гордона $\omega_{x y}=\sin \omega$ возникло в геометрии в статье П. Л. Чебьшёва “О кройке одежды” [46] в 1878 году.

Э.Г. Позняк доказал [136], [137], что каждое решение $\omega(x, y)$ уравнения синус Гордона порождает в $E^{3}$ поверхность с гауссовой кривизной $K=-1$, на которой $\omega(x, y)$ - угол между асимптотическими линиями; $x, y$ - натуральные параметры на них; там, где $\sin \omega=0$, поверхность имеет особые точки. И другие солитонные уравнения в частности, уравнение Кортевега-де Фриза

$$
u_{t}+6 u u_{x}+u_{x x x}=0
$$

модифицированное уравнение Кортевега-де Фриза

$$
u_{t}+6 u^{2} u_{x}+u_{x x x}=0
$$

- возникают на поверхностях постоянной отрицательной кривизны. А именно, при специальном выборе систем координат $(x, t)$ на поверхности постоянной отрищательной кривизныгеодезические кривизны $u(x, t)$ семейства координатных линий $t=\mathrm{const}$ удовлетворяют указанным выше уравнениям [49], [50], [148]. Таким же образом возникает и уравнение синус Гордона и другие солитонные уравнения.

Для изометрического погружения двумерных метрик постоянной гауссовой кривизны $c$ в трехмерное пространство постоянной кривизны $\widetilde{c}, c \neq \widetilde{c}$, условия интегрируемости сводятся к sin- и sinh-Гордона уравнениям. При изометрическом погружении 
$M^{l}(c)$ в $M^{l+p}(\widetilde{c})$ уравнения интегрируемости Гаусса, Кодацци, Риччи рассматриваются как многомерное обобщение $\sin -Г$ Гордона уравнения. При с $<0, \widetilde{c}=0$ уравнения Кодащи-Риччи имеют своим условием интегрируемости уравнение Гаусса. Поэтому при $c=-1, \widetilde{c}=0, p=l-1$ вся система погружений сводится к системе (3.2.9). Используя систему солитонов, были даны изометрические погружения конечного типа $f: M^{l}(c) \rightarrow M^{l+p}(\widetilde{c}), c \neq 0 \neq \widetilde{c}, c \neq \widetilde{c}$, с плоской нормальной связностью [71].

Главньй вклад классических геометров в теорию солитонов заключается в построении явных решений солитонных уравнений [164].

В 1883 году Бэклунд ввел преобразование, которое по одной поверхности постоянной отрицательной кривизны в $E^{3}$ строит другую поверхность постоянной отрицательной кривизны.

Отображение Беклунда между двумя поверхностями $F_{1}$ и $F_{2}$ обладает тем свойством, что отрезок, соединяюший соответствуюшие точки, - касательньй к обеим поверхностям. Если 1) отрезки имеют постоянную длину и 2) угол между нормалями в соответствуюших точках постоянен, то поверхности $F_{1}$ и $F_{2}$ имеют постоянную отрицательную гауссову кривизну.

Преобразование Беклунда для многомерного случая погружения пространства Лобачевского $L^{l}$ в $E^{2 l-1}$ было введено и изучено К. Тененблат и С. Тернгом [161]. Оно дает возможность по заданному подмногообразию $F^{l} \subset E^{2 l-1}$ постоянной отрицательной кривизны строить новое подмногообразие $\widetilde{F}^{l} \subset E^{2 l-1}$ постоянной отрищательной кривизны. Тем самым, по одному солитонному решению системы (3.2.9) мы строим новое решение. При этом асимптотические линии переходят в асимптотические линии.

Пусть для многомерных подмногообразий $F_{1}^{l}$ и $F_{2}^{l}$ в $E^{2 l-1}$ вьполняется условие касания. Если условие 1) остается, а условие 2) заменяется условием, что изоклинические углы между нормальными пространствами в соответствуюших точках постоянны, то подмногообразия $F_{1}^{l}, F_{2}^{l}$ в $E^{2 l-1}$ имеют постоянную отрицательную секционную кривизну. При этом преобразовании линии кривизны и асимптотические линии также соответствуют друг другу [162].

Система, аналогичная системе (3.2.9), для подмногообразий постоянной секционной кривизны в псевдоримановых пространственных формах рассматривалась в [15], а преобразование Беклунда для этой системы в [43].

Многомерное обобщение преобразования Бианки дано в [8] Ю. А. Аминовым. Пусть $F^{l}-$ поверхность постоянной отрицательной секционной кривизны в $E^{2 l-1}$. Пусть $u=\left(u_{1}, \ldots, u_{l}\right)$ - полугеодезические координаты на $F^{l}$ такие, что метрика $F^{l}$ записьвается в виде:

$$
d s^{2}=d u_{l}^{2}+e^{2 u_{l}}\left(d u_{1}^{2}+\cdots+d u_{l-1}^{2}\right) .
$$

Тогда преобразование Бианки ставит в соответствие точке с радиус-вектором $r=$ $r(u)$ точку

$$
\bar{r}=r-\frac{\partial r}{\partial u_{l}} .
$$

Преобразование Бианки, так же как и преобразование Беклунда, подмногообразие $F^{l}$ постоянной отрицательной кривизны в $E^{2 l-1}$ переводит в подмногообразие той же постоянной отрицательной кривизны [8]. И по известному решению солитонной системы 
(3.2.9) строится ее новое решение. Преобразования Бианки рассматривались также в [115].

5.2. Изометрические погружения с дополнительными ограничениями. Если $f: M^{l}(c) \rightarrow M^{l+p}$ есть изометрическое погружение в $M^{l+p}(\widetilde{c})$ с плоской нормальной связностью и минимально, то подмногообразие $f\left(M^{l}(c)\right)$ есть часть тора Клиффорда, стандартно вложенного в сферу $S^{2 l-1}$ [51], [117]. Если условие минимальности заменим на параллельность вектора средней кривизны, то

1) при $c=\widetilde{c}$ или $f$ есть вполне геодезическое, или $c=0$ и

$$
f\left(M^{l}\right) \subset S^{1}\left(r_{1}\right) \times \cdots \times S^{1}\left(r_{p}\right) \times E^{l-p} \subset E^{l+p} ;
$$

2) при $\widetilde{c}>c=0$

$$
f\left(M^{l}\right) \subset S^{1}\left(r_{1}\right) \times \cdots \times S^{1}\left(r_{l}\right) \times S^{2 l-1}(\widetilde{c}) \subset E^{2 l},
$$

где $r_{1}^{2}+\cdots+r_{l}^{2}=1 / \widetilde{c}$

3) при $\tilde{c}<c=0$

$$
f\left(M^{l}\right) \subset H^{1}\left(r_{1}\right) \times S^{1}\left(r_{2}\right) \times \cdots \times S^{1}\left(r_{l}\right) \subset H^{2 l-1}(\widetilde{c}) \subset E_{1}^{2 l},
$$

где $-r_{1}^{2}+r_{2}^{2}+\cdots+r_{l}^{2}=1 / \widetilde{c}$

4) $f=i \circ f^{\prime}$, где $f^{\prime}$ имеет тип 1), 2) или 3) и $i$ есть омбилическое или вполне геодезическое включение [56].

Лагранжевы изометрические вложения вешественной пространственной формы $M^{l}(c)$ в комплексную пространственную форму $\widetilde{M}^{l}(4 c)$ определены в [47].

Полностью классифицированы лагранжевы минимальные подмногообразия постоянной секционной кривизны $c \neq \widetilde{c}$ в комплексной индефинитной пространственной форме $\widetilde{M}_{1}^{n}(4 \widetilde{c})$ постоянной голоморфной кривизны $4 \widetilde{c}[106]$. В комплексной римановой пространственной форме это получено в [64]. В [121] определены все локальные изометрические погружения в комплексные пространственные формы комплексных римановых пространственных форм постоянной голоморфной кривизны как кэлеровых подмногообразий.

Tеорема 5.2.1 (Накагава, Огиу [121]). Пусть $F^{l}(c)$ есть кәлерово подмногообразие постоянной голоморфной кривизны $\widetilde{c}$ в комплексной пространственной форме $M^{l+p}(\widetilde{c})$ постоянной голоморфной кривизны $\widetilde{c}$.

1) Если $\widetilde{c}>0$ и вложение является полныц (т.е. не лежит во вполне геодезическом подпространстве меньшей размерности), то $\widetilde{c}=\nu c$ u $l+p=$ $\left(\begin{array}{c}l+\nu \\ \nu\end{array}\right)-1$, где $\nu$ - натуральное число.

2) Если $\widetilde{c} \leqslant 0$, то $\widetilde{c}=c$ и подмногообразие $F^{l}(c)$ является вполне геодезическим подмногообразием в $M^{l+p}(\widetilde{c})$. 


\section{Некоторые нерешенные вопросы}

1. Классические результаты об однозначной определенности сферы $S^{2}$ в $E^{3}$ и о цилиндричности полной поверхности нулевой гауссовой кривизны в $E^{3}$ верны в классе гладких класса $C^{1}$ поверхностей ограниченной внешней кривизны, а также в классе гладких класса $C^{1}$ аффинно устойчивых погружений (глава 1 ).

Однако не известно, верна ли теорема Гильберта о невозможности изометрического погружения плоскости Лобачевского в евклидово пространство $E^{3}$ в классе гладких поверхностей класса $C^{1}$ локально ограниченной внешней кривизны [141].

В последнее время активно исследовалась внутренняя геометрия пространств Александрова, которые являются нерегулярным обобщением римановых и финслеровых пространств. Однако нерегулярные аналоги во внешней геометрии подмногообразий не получили развития, хотя можно ввести определение подмногообразий класса $C^{1}$ ограниченной внешней кривизны для произвольной коразмерности и доказать аналоги многих теорем в классе этих подмногообразий, а также в классе аффинно устойчивых погружений.

2. В [159] полно изложены вопросы изометрического погружения полных двумерных метрик постоянной кривизны в односвязные пространства $M^{3}$ постоянной кривизны (раздел 2.2). И там отмечено, что неизвестно, существуют ли в пространстве Лобачевского $H^{3}(-1)$ полные регулярные поверхности с внешней кривизной $0<K_{\mathrm{ext}}<1$ (или, что равносильно, с гауссовой кривизной $-1<K<0$ ), отличные от эквидистант и поверхностей врашения.

3. В [134] дано явное задание изометрических погружений полных двумерных метрик в евклидовы пространства с минимальной коразмерностью (раздел 2.3). Всего сушествует 10 плоских трехмерных пространственных форм. Естественно это же сделать для них.

4. В разделе 2.3 описано локальное строение изометрических погружений плоских двумерных метрик в евклидово пространство $E^{4}$ поверхностями с плоской нормальной связностью. Выяснить глобальное строение компактных регулярных двумерных поверхностей нулевой гауссовой кривизны и с плоской нормальной связностью.

ПрЕДПОЛОЖЕниЕ. Это либо плоские торы в сферическом пространстве $S^{3}$, либо декартово метрическое произведение двух плоских замкнутых кривых, которые лежат в дополнительных ортогональных плоскостях.

Аналогичный результат должен иметь место для компактных подмногообразий $F^{l}$ нулевой секционной кривизны с плоской нормальной связностью в $E^{2 l}$.

5. Найти все двумерные поверхности постоянной положительной гауссовой кривизны, у которых все внутренние движения индуцируются движениями объемлющего евклидова пространства.

ПРЕДПОЛОЖЕниЕ. Это только стандартные изометрические погружения с радиус-вектором, состояшим из ортонормированного базиса собственных функций оператора Лапласа на сфере, отвечаюших одному и тому же собственному значению.

6. Известно (теорема Томпкинса), что не сушествует компактного плоского подмногообразия $F^{l}$ в евклидовом пространстве $E^{l+p}, p<l$ (следует из теоремы 3.1.1). 
Пусть $M^{l}, M^{l+p}$ - две плоские пространственные формы. Оценить, в зависимости от рангов фундаментальных груп, коразмерность, при которой невозможно изометрическое погружение.

ПРЕДПОЛОЖЕНИЕ. Пусть $r_{1}-$ ранг $\pi_{1}\left(M^{l}\right), r_{2}-$ ранг $\pi_{2}\left(M^{l+p}\right)$. Если $p<r_{1}-r_{2}$, то невозможно изометрическое погружение $M^{l}$ в $M^{l+p}$.

7. Полная поверхность $F^{l}$ нулевой секционной кривизны в $E^{l+p}$ является цилиндром с $(l-p)$-мерной образующей (теорема 3.1.1).

Обобшить эту теорему на плоские пространственные формы. Если $M^{l}, M^{l+p}$ компактные и $f: M^{l} \rightarrow M^{l+p}$ есть изометрическое погружение, то локально $M^{l}$ есть метрическое произведение, $M^{l}=M^{k} \times E^{l-k}, k \leqslant p$, где $E^{l-k}$ - евклидов фактор.

ПРЕДПоложениЕ. Существует конечнократная накрьвающая $\widetilde{M}^{l}$ такая, что $\widetilde{M}$ есть декартово произведение $\widetilde{M}^{l}=\widetilde{M}^{k} \times T^{l-k}$, где $\widetilde{M}^{k}-$ компактное многообразие, $T^{l-k}-$ тор.

Будет ли $\widetilde{M}^{l}$ метрическим произведением, где $\widetilde{M}^{k}, T^{l-k}-$ плоские многообразия?

Аналогичный результат должен иметь место и в случае некомпактных плоских пространственных форм, где размерность тора зависит от рангов фундаментальных групп $M^{l}, M^{l+p}$.

8. Полная неодносвязная гиперболическая пространственная форма не допускает регулярного изометрического погружения в $E^{2 n-1}$ (следствие теоремы 3.2.8).

Многомерная проблема Гильберта: доказать это утверждение в односвязном случае, т.е. доказать, что невозможно регулярное изометрическое погружение пространства Лобачевского $H^{l}$ в евклидово пространство $E^{2 l-1}$.

Аналогичная проблема имеет место, когда объемлющее пространство есть пространство Лобачевского.

9. Л. Бибербах построил такое геометрическое погружение плоскости Лобачевского в гильбертово пространство, что все движения плоскости Лобачевского индуцируются движениями гильбертова пространства [17]. Такое погружение плоскости Лобачевского невозможно ни в какое конечномерное евклидово пространство [98].

Д. Блануша построил изометрическое вложение пространства Лобачевского $H^{n}$ в гильбертово пространство [18].

Индуцируются ли движения пространства Лобачевского $H^{n}$ движениями гильбертова пространства?

10. Дж. Мур доказал, что компактное подмногообразие $F^{l}$ постоянной положительной секционной кривизны в $E^{l+p}$ односвязно, т.е. изометрично стандартной сфере, если $p \leqslant l / 2$ (теорема 3.3.3).

ПРЕДПОЛОЖЕНИЕ. Этот результат верен при $p<l$, а может быть, при $p \leqslant l$.

11. Дж. Мур доказал, что изометрическоепогружение стандартной сферы $S^{l}$ в $E^{l+2}$ продолжается до изометрического погружения шара $D^{l+1}$, ограниченного сферей $S^{l}$, в $E^{l+1}$ (теорема 3.3.4). Обобшить эту теорему на случай, когда сфера $S^{l}$ заменяется компактной гиперповерхностью $F^{l} \subset E^{l+1}$ положительной секционной кривизны. Из условий на кривизну следует вьпуклость гиперповерхности $F^{l}$. Она ограничивает область $\Omega^{l+1}$, диффеоморфную шару; гиперповерхность $F^{l}$ диффеоморфна сфере. 
12. Найти все (неприводимые) изометрические погружения многомерных (больше двумерных) метрик постоянной положительной секционной кривизны в евклидово пространство (и другие пространства постоянной кривизны) такие, что все внутренние движения индуцируются движениями объемлющего пространства. Это связано с теоремой До Кармо и Валлах [61] о минимальном изометрическом погружении сфер в сферы (п. 3.3.3). И, наверное, в общем случае эти погружения не будут исчерпьваться стандартными погружениями, где координатньми функциями являются все собственные функции, отвечающие одному и тому же собственному значению оператора Лапласа на сфере. Выделить естественные геометрические условия, когда погружение будет стандартньм.

13. Для гиперповерхностей Л. Грейвс доказал обобшение теоремы Хартмана-Ниренберга для изометрических погружений псевдоевклидова (лоренщева) пространства $E_{1}^{l}$ в $E_{1}^{l+1}$ (теорема 4.1.1).

Доказать аналог теоремы 3.1.1 для изометрического погружения псевдоевклидова пространства $E_{s}^{l}$ в $E_{s}^{l+p}$.

Частньй результат в этом направлении есть теорема 4.1.2.

14. Пусть $H_{s}^{l}$ - геодезически полное псевдориманово многообразие постоянной отрицательной кривизны. Если $s \neq 0,1,3,7$, то многообразие $H_{s}^{l}$ не погружается изометрично в псевдоевклидово пространство $E_{s}^{2 l-1}$ (теорема 4.2.5).

Если $s=0$, то $H_{0}^{l}=H^{l}$ и это задача 8.

Предполагается такой же отрицательный ответ и при $s=1,3,7$. При $s=3,7$ решение можно получить из топологических препятствий, при $s=1$-из метрических препятствий, как и в случае $s=0$.

15. И.Х. Сабитовым доказано, что любая заданная в круге локально евклидова метрика изометрично вкладьвается в $E^{3}$ (теорема 2.3.3). Естественно поставить аналогичный вопрос для плоских метрик, заданных в $n$-мерном шаре, $n \geqslant 3$ (М. Громов [79]).

\section{СПИСОК ЛИТЕРАТУРЫ}

[1] K. Abe. Isometric immersions of $H_{1}^{n}$ into $H_{1}^{n+1}$ // Pacific J. Math. 1996. V. 173. № 1. P. 1-27.

[2] K. Abe, A. Haas. Isometric Immersions of $H^{n}$ into $H^{n+1} / /$ Proc. Sympos. Pure Math. 1993. V. 54. № 3. P. 23-30.

[3] K. Abe, M. A. Magid. Relative nullity foliations and indefinite isometric immersions // Pacific J. Math. 1986. V. 124. P. 1-20.

[4] K. Abe, M. A. Magid. Indefinite Kahler submanifolds with positive index of relative nullity // Ann. Global Anal. Geom. 1988. V. 6. № 3. P. 231-258.

[5] K. Abe, H. Mori, H. Takanashi. A parametrization of isometric immersions between hyperbolic spaces // Geom. Dedicata. 1997. V. 65. P. 31-46.

[6] S. Alexander, E. Portnoy. Cylindricity of isometric immersions between hyperbolic spaces // Trans. Amer. Math. Soc. 1978. V. 237. P. 311-329.

[7] Ю.А. Аминов. О погружении областей $n$-мерного пространства Лобачевского в $(2 n-1)$-мерное евклидово пространство // Докл. АН СССР. 1977. Т. 236. № 3. С. 521-524.

[8] Ю. А. Аминов. Преобразование Бианки для многомерного пространства Лобачевского // Укр. геом. сб. Харьков. 1978. № 21. С. 3-5.

[9] Ю.А. Аминов. Изометрические погружения $n$-мерного пространства Лобачевского в $(2 n-1)$-мерное евклидово пространство // Матем. сб. 1980. Т. 111. №3. С. 402-433. 
[10] Ю.А. Аминов. Проблемы вложения: геометрические и топологические аспекты // Итоги науки и техники. Проблемы геометрии. Т. 13. М.: ВИНИТИ, 1982. С. 119-157.

[11] Ю. А. Аминов. Изометрические погружения области в 3-мерном пространстве Лобачевского $L^{3}$ в евклидово пространство $E^{5}$ и движение твердого тела // Матем. сб. 1983. T. $122(164)$. C. $12-30$.

[12] Ю. А. Аминов. Изометрические погружения с плоской нормальной связностью областей $n$-мерного пространства Лобачевского в евклидово пространство. Модели теории поля // Матем. сб. 1988. Т. 137 (179). № 3. С. 275-299.

[13] Ю. А. Аминов. Геометрия грассманова образа локально изометрического погружения $n$-мерного пространства Лобачевского в $(2 n-1)$-мерное евклидово пространство // Матем. сб. 1997. Т. 188. №1. С. 1-27.

[14] Д. Г. Азов. Погружение методом Д. Блануши некоторых классов полных $n$-мерных римановых метрик в евклидовы пространства // Вестник МГУ. Сер. Матем. 1985. №5. C. $70-74$.

[15] J. L. Barbosa, W. Ferreira, K. Tenenblat. Submanifolds of constant sectional curvature in pseudo-Riemannian manifolds // Ann. Global Anal. Geom. 1996. V. 14. № 4 . P. 381-401.

[16] J. Bernard. A non immersion result for lens spaces $L^{n}\left(Z^{m}\right) / /$ Math. J. Okayama Univ. 1995. V. 37. P. $137-151$

[17] L. Bieberbach. Eine singularitätenfreie Fläche konstanter negativer Krümmung im Hilbertschen Raum // Comment. Math. Helv. 1932. V. 4. P. 248-255.

[18] D. Blanusha. Eine isometrische und singularitäten freie Einbettung des $n$-dimensionalen hyperbolischen Raumes im Hilbertschen Raum // Monatsh. Math. 1953. V. 57. № 2 . P. $102-108$.

[19] D. Blanusha. Les espaces elliptiques plongés isométriquement dans des espaces euclidiens // Glas. Mat.-Fiz. i Astron. 1953. V. 8. № 1. P. 3-23.

[20] D. Blanusha. Les espaces elliptiques plongés isométriquement dans des espaces euclidiens // Glas. Mat.-Fiz. i Astron. 1953. V. 8. № 2. P. 81-114.

[21] D. Blanusha. Le plan elliptique plongé isométriquement dans un espace à quatre dimensions ayant une courbure constante // Glas. Mat.-Fiz. i Astron. 1954. V. 9. № 1. P. 41-58.

[22] D. Blanusha. Le plongement isométrique de la bande de Mobius infiniment large euclidienne dans un espace sphérique, parabolique ou hyperbolique à quarte dimensions // Bull. Internat. Acad. Yougosl. Sci. et Beaux-Arts. 1954. № 12. P. 19-23.

[23] D. Blanusha. Über die Einbettung hyperbolischer Räume in euklidische Räume // Monatsh. Math. 1955. V. 59. № 3. P. 217-229.

[24] D. Blanusha. $C^{\infty}$-isometric imbeddings of cylinders with hyperbolic metric in Euclidean 7-space // Glas. Mat.-Fiz. i Astron. 1956. V. 11. № 3-4. P. 243-246.

[25] D. Blanusha. A simple method of imbedding elliptic spaces in Euclidean spaces // Glas. Mat.-Fiz. i Astron. 1957. V. 12. № 3. P. 190-200.

[26] D. Blanusha. $C^{\infty}$-isometric imbeddings of the hyperbolic plane and of cylinders with hyperbolic metric in spherical space // Ann. Mat. Pura Appl. 1962. V. 57. P. 321-337.

[27] А. А. Борисенко. Строение поверхностей с вырожденньм сферическим изображением // Автореферат дисс. . . . канд. физ.-матем. наук. Харьков: Харьков. гос. ун-т, 1973.

[28] А. А. Борисенко. О классе римановых пространств строго отрицательной кривизны // Укр. геом. сб. Харьков. 1973. № 13. С. 15-18.

[29] А.А.Борисенко. О строении непрерывной поверхности, содержащей прямую // Укр. геом. сб. Харьков. 1973. №14. С. 21-24.

[30] А.А. Борисенко. О полных поверхностях в пространствах постоянной кривизны // Укр. геом. сб. Харьков. 1974. №15. С. 8-15.

[31] А. А. Борисенко. О компактных поверхностях отрицательной внешней кривизны в римановом пространстве // Укр. геом. сб. Харьков. 1976. № 19. С. 9-11.

[32] А. А. Борисенко. Об изометрическом погружении псевдоримановых пространств постоянной кривизны // Укр. геом. сб. Харьков. 1976. № 19. С. 11-18. 
[33] А. А. Борисенко. Поверхности неположительной внешней кривизны в пространстве постоянной кривизны // Матем. сб. 1981. Т. 114 (156). № 3. С. 339-354.

[34] А. А. Борисенко. О полных параболических поверхностях в евклидовом пространстве // Укр. геом. сб. Харьков. 1985. № 28. С. 8-19.

[35] А. А. Борисенко. О цилиндрических многомерных поверхностях в пространстве Лобачевского // Укр. геом. сб. Харьков. 1990. №33. С. 18-27.

[36] А. А. Борисенко. Внешняя геометрия сильно параболических многомерных подмногообразий // УМН. 1997. Т. 52. №6. С. 3-52.

[37] А. А. Борисенко. Внешняя геометрия параболических и седловых многомерных подмногообразий // УМН. 1998. Т. 53. №6. С. 3-52.

[38] A. Borisenko, M.L. Rabelo, K. Tenenblat. Strongly parabolic timelike submanifolds of Minkovsky space // Matematicheskaya Fizika, Analiz, Geometriya. Kharkov. 1999. V. 6. № 1-2. P. 10-21.

[39] R. Bryant. Minimal surfaces of constant curvature in $S^{4} / /$ Trans. Amer. Math. Soc. 1985. V. 290. № 1. P. 259-271.

[40] Ю. Д. Бураго. Геометрия поверхностей в евклидовых пространствах // Совр. пробл. матем. Фундаментальные направления. Геометрия-3. Т. 48. М.: ВИНИТИ, 1989. С. 5-97.

[41] А. В. Бушмелев. Изометрические вложения бесконечного плоского листа Мёбиуса и плоской бутылки Клейна // Вестник МГУ. Сер. матем., мех. 1988. № 3. С. 38-41.

[42] E. Calabi. Minimal immersions of surfaces in Euclidean spheres // J. Differential Geom. 1967. V. 1. P. 111-125.

[43] P. T. Campos, K. Tenenblat. Backlund transformations for a class of systems of differential equations // Geom. Funct. Anal. 1994. V. 4. № 3. P. 270-287.

[44] E. Cartan. Sur les variétés de courbure constante d'un espace euclidien ou non euclidien // Bull. Soc. Math. France. 1919. V. 47. P. 125-160; 1920. V. 48. P. 32-208.

[45] E. Cartan. Sur la possibilité de plonger un espace riemannien donné dans un espace euclidien // Ann. Soc. Polon. Math. 1927. V. 6. P. 1-7.

[46] П. Л. Чебышёв. Sur la coupe des habits // Доклад во Франц. Ассоциации содейств. преподаванию наук 28.08.1878 [Association Française pour l'avancement des sciences]. Pyc. перевод: О кройке одежды // УМН. 1946. Т. 1. № 2. С. 38-42.

[47] B.-Y. Chen, F. Dillen, L. Verstraelen, L. Vrancken. Lagrangian isometric immersions of a real-space form $M^{n}(c)$ into a complex-space-form $\widetilde{M}^{n}(4 c) / /$ Math. Proc. Cambridge Philos. Soc. 1998. V. 124. № 1. P. 107-125.

[48] S.S. Chern, N.H. Kuiper. Some theorems on the isometric imbedding of compact Riemannian manifolds in Euclidean space // Ann. of Math. (2). 1952. V. 56. P. 422-430.

[49] S.S. Chern, K. Tenenblat. Foliations on a surface of constant curvature and the modified Korteweg-de Vries equations // J. Differential Geom. 1981. V. 16. № 3. P. 347-349.

[50] S.S. Chern, K. Tenenblat. Pseudospherical surfaces and evolution equations // Stud. Appl. Math. 1986. V. 74. № 1. P. 55-83.

[51] M. Dajczer. Reduction of codimension of regular isometric immersions // Math. Z. 1982. V. 179. P. 263-286.

[52] M. Dajczer, P. Dombrowski. Examples of 1-codimensional non totally geodesic isometric immersions of pseudo-Riemannian space forms with the same positive constant curvature and the same space-like rank // Proceedings of the conference on Global Differential Geometry and Global Analysis (Berlin, 1984). Berlin: Springer-Verlag 1985. P. 59-73. (Lecture Notes in Math. V. 1156.)

[53] M. Dajczer, S. Fornari. Isometric immersions between indefinite Riemannian spheres // Yokohama Math. J. 1987. V. 35. P. 61-69.

[54] M. Dajczer, L. Rodriguez. Rigidity of codimension two indefinite spheres // Geom. Dedicata. 1990. V. 34. № 3. P. 243-248.

[55] M. Dajczer, R. Tojeiro. On composition of isometric immersions // J. Differential Geom. 1992. V. 36. P. 1-18.

[56] M. Dajczer, R. Tojeiro. Submanifolds of constant sectional curvature with parallel or constant mean curvature // Tôhoku Math. J. (2). 1993. V. 45. № 1. P. 43-49. 
[57] M. Dajczer, R. Tojeiro. On flat surfaces in space forms // Houston J. Math. 1995. V. 21. № 2. P. 319-338.

[58] M. Dajczer, M. Antonucci, G. Oliveira, P. Lima-Filho, R. Tojeiro. Submanifolds and Isometric Immersions. Houston: Publish or Perish, Inc., 1990.

[59] D. Deturk, W. Ziller. Spherical minimal immersions of spherical space forms // Proc. Sympos. Pure Math. 1993. V. 54. № 3. P. 111-120.

[60] M. Do Carmo, M. Dajczer. Local isometric immersions of $R^{2}$ into $R^{4} / /$ J. Reine Angew. Math. 1993. V. 442. P. 205-219.

[61] M. Do Carmo, N.R. Wallach. Minimal immersion of spheres into spheres // J. Differential Geom. 1970. V. 4. P. 91-104.

[62] Н. В. Ефимов. Непогружаемость полуплоскости Лобачевского // Вестник МГУ. Сер. матем., мех. 1975. № 2. С. 83-86.

[63] N. Ejiri. Total real submanifolds in a 6-sphere // Proc. Amer. Math. Soc. 1981. V. 83. P. $759-763$.

[64] N. Ejiri. Total real minimal immersions of $n$-dimensional real space forms into $n$-dimensional complex space forms // Proc. Amer. Math. Soc. 1982. V. 84. P. 243-246.

[65] K. Enomoto. Global properties of the Gauss image of flat surfaces in $R^{4} / /$ Kodai Math. J. 1987. V. 10. P. 272-284

[66] J. Erbacher. Isometric immersions of constant mean curvature and triviality of the normal connections // Nagoya Math. J. 1972. V. 45. P. 139-165.

[67] C. M. Escher. Minimal isometric immersions of inhomogeneous spherical space forms into spheres. A necessary condition for existence // Trans. Amer. Math. Soc. 1996. V. 348. № 9. P. 3713-3732.

[68] C. M. Escher. Rigidity of minimal isometric immersions of spheres into spheres // Geom. Dedicata. 1998. V. 73. P. 275-293.

[69] D. Ferus. On isometric immersions between hyperbolic spaces // Math. Ann. 1973. V. 205. P. 193-200.

[70] D. Ferus. Isometric immersions of constant curvature manifolds // Math. Ann. 1975. V. 217. P. $155-156$.

[71] D. Ferus, F. J. Pedit. Isometric immersions of space forms and soliton theory // Math. Ann. 1996. V. 305. P. 1-14.

[72] D. Ferus, U. Pinkall. Constant curvature 2-spheres in the 4-sphere // Math. Z. 1989. V. 200. P. 269-271.

[73] J. A. Galvez, A. Martinez, R. Milan. Flat surfaces in the hyperbolic 3-spaces // Math. Ann. 2000. V. 316. P. 419-439.

[74] Талеб Гарибе. Поверхности в $E^{4}$ с постоянной внешней и внутренней геометрией // Укр. геом. сб. Харьков. 1981. № 24. С. 12-18.

[75] Талеб Гарибе. Двумерные поверхности с постоянной внешней геометрией в $E^{5} / / \mathrm{У}_{\text {кр. }}$ геом. сб. Харьков. 1982. № 25. С. 11-22.

[76] L.K. Graves. Codimension one isometric immersions between Lorentz spaces // Trans. Amer. Math. Soc. 1979. V. 252. P. 367-392.

[77] L. K. Graves, K. Nomizu. Isometric immersions of Lorentzian space forms // Math. Ann. 1978. V. 233. P. 125-136.

[78] Ph.A. Griffiths, G. R. Jensen. Differential Systems and Isometric Embeddings. Princeton: Princeton Univ. Press, 1987. (Ann. of Math. Stud. V. 114.)

[79] М. Л. Громов. Дифференциальные соотношения с частными производными. М.: Мир, 1990.

[80] М.Л. Громов, В.А. Рохлин. Вложения и погружения в римановой геометрии // УМH. 1970. T. 25. № 5. C. 3-62.

[81] P. Hartman. On the isometric immersions in Euclidean space of manifolds with nonnegative sectional curvature // Trans. Amer. Math. Soc. 1970. V. 147. № 2. P. 529-540.

[82] P. Hartman, L. Nirenberg. On spherical image maps whose jacobians do not change sign // Amer. J. Math. 1959. V. 81. P. 901-920. 
[83] Ph. Hartman, A. Wintner. On the asymptotic curves of a surfaces // Amer. J. Math. 1951. V. 73. № 1. P. 149-172.

[84] W. Henke. Riemannsche Mannigfaltigkeit konstanter positiver Krümmung in euklidischen Räumen der Kodimension 2 // Math. Ann. 1971. V. 193. № 4. P. 265-278.

[85] W. Henke. Über die Existenz isometrischer Immersionen der Kodimension 2 von sphärischen Raumformen in Standard-Raüme // Math. Ann. 1976. V. 222. P. 89-95.

[86] W. Henke. Isometrische Immersionen des $n$-dim. hyperbolischen Raumes $H^{n}$ in $E^{4 n-3}$ // Manuscripta Math. 1981. V. 34. P. 265-278.

[87] W. Henke. Isometric immersions of $n$-dim. hyperbolic spaces into $(4 n-3)$-dim. standart spheres and hyperbolic spaces // Math. Ann. 1982. V. 258. P. 341-348.

[88] D. Hilbert. Über Flächen von konstanter Gaüs'shen Krümung // Trans. Amer. Math. Soc. 1901. № 2. P. 87-99.

[89] J. Hong, C. Zuilly. Isometric imbedding of the 2-sphere with nonnegative curvature in $E^{3} / /$ Math. Z. 1995. V. 219. P. 323-334.

[90] Hu Ze-Jun. Isometric immersions of the hyperbolic space $H^{n}(-1)$ into $H^{n+1}(-1) / /$ Colloq. Math. 1999. V. 79. № 1. P. 17-23.

[91] Hu Ze-Jun, Zhao Guo-Song. Classification of isometric immersions of the hyperbolic space $H^{2}$ into $H^{3} / /$ Geom. Dedicata. 1997. V. 65. №1. P. 47-57.

[92] Hu Ze-Jun, Zhao Guo-Song. Isometric immersions from the hyperbolic space $H^{2}(-1)$ into $H^{3}(-1)$ // Proc. Amer. Math. Soc. 1997. V. 25. № 9. P. 2693-2697.

[93] А. Б. Иванов. Изометрическое погружение полных двумерных локальных евклидовых многообразий в евклидовы пространства // Матем. заметки. 1973. Т. 13. № 3. С. 427-429.

[94] H. Jacobowitz. Implicit function theorems and isometric embeddings // Ann. of Math. (2). 1972. V. 95. P. 191-225.

[95] I. M. James. Two problems studied by Heinz Hopf // Lecture Notes in Math. 1972. V. 279. P. $135-160$.

[96] M. Janet. Sur la possibilite de plonger un espace riemannien donne dans un espace euclidien // Ann. Soc. Polon. Math. 1926. V. 5. P. 38-43.

[97] С. Б. Кадомцев. Невозможность некоторых специальных изометрических погружений пространств Лобачевского // Матем. сб. 1978. Т. 107. № 2. С. 175-198.

[98] С.Б.Кадомцев. Об одном свойстве группы движений пространства Лобачевского // Матем. заметки. 1984. Т. 35. № 1. С. 107-110.

[99] С. Б. Кадомцев, Э.Г. Позняк, Д. Д. Соколов. Некоторые вопросы геометрии Лобачевского, связанные с физикой // Итоги науки и техники. Проблемы геометрии. Т. 13. М.: ВИНИТИ, 1982. С. 157-188.

[100] Ж. Кайдасов. О регулярном изометрическом погружении в $E^{3}$ некоторых некомпактных частей плоскости Лобачевского // Автореферат дисс. . . . канд. физ.-матем. наук. М.: МГУ, 1987.

[101] Ж. Кайдасов, Е. В. Шикин. Об изометрическомпогружении в $E^{3}$ вьпуклой области плоскости Лобачевского, содержащей два орикруга // Матем. заметки. 1986. Т. 39. № 4. C. $612-617$.

[102] K. Kenmotsu. On minimal immersions of $R^{2}$ into $S^{n} / /$ J. Math. Soc. Japan. 1976. V. 28. P. $182-191$.

[103] K. Kenmotsu. Minimal surfaces with constant curvature in 4-dimensional space forms // Proc. Amer. Math. Soc. 1983. V. 89. P. 133-138.

[104] Y. Kitagawa. Embedded flat tori in the unit 3-sphere // J. Math. Soc. Japan. 1995. V. 47. № 2. P. 275-296.

[105] T. Klotz-Milnor. Efimov's theorem about complete immersed surfaces of negative curvature // Adv. Math. 1972. V. 8. № 3. Р. 474-543. Рус. перев.: Т. Клотц-Милнор. Tеорема Ефимова о полных погруженных поверхностях отрицательной кривизны // УМН. 1986. T. 41. № 5. C. 3-57.

[106] M. Kriele, L. Vrancken. Minimal Lagrangian submanifolds of Lorentzian complex space forms with constant sectional curvature // Arch. Math. 1999. V. 72. P. 223-232. 
[107] N. H. Kuiper. On $C^{1}$-isometric imbeddings // Proc. Konink. Nederl. Akad. Wetensch. 1955. V. А-58. Р. 545-556. Рус. перев.: Н. О. Кейпер. О $C^{1}$-изометрических вложениях // Математика. 1957. Т. 1. № 2. С. 17-28.

[108] H. Lawson, S.T. Yau. Compact manifolds nonpositive curvature // J. Differential Geom. 1972. V. 6. № 1-2. P. 211-229.

[109] J. Levine. Imbedding and immersion of real projective spaces // Proc. Amer. Math. Soc. 1963. V. 14. P. 801-803.

[110] C.S. Lin. The local isometric embedding in $E^{3}$ of two-dimensional Riemannian manifolds with non-negative curvature // J. Differential Geom. 1985. V. 21. № 2. P. 213-230.

[111] А. Е. Либер. О классе римановых пространств постоянной отрицательной кривизны // Уч. зап. Саратов. гос. ун-та. Сер. физ.-матем. 1938. Т. 1. № 2. С. 105-122.

[112] В. Т. Лисица. Об $n$-мерных геликоидальных поверхностях в евклидовом пространстве $E^{n} / /$ Матем. заметки. 1987. Т. 41. № 4. С. 549-556.

[113] R. Maltz. The nullity spaces of constant curvature like tensors // J. Differential Geom. 1972. V. 7. № 3-4. P. 219-299.

[114] Л. А. Масальцев. О геликоидальных поверхностях в евклидовом пространстве // Укр. геом. сб. Харьков. 1983. № 26. С. 100-103.

[115] Л. А. Масальцев. Псевдосферические конгруэнции Бианки в $E^{2 n-1} / /$ Математическая физика, анализ, геометрия. 1994. Т. 1. № 3/4. С. 505-513.

[116] Л. А. Масальцев. О минимальных подмногообразиях постоянной кривизны в евклидовом пространстве // Изв. вузов, сер. матем. 1998. № 9 (436). С. 64-65.

[117] J. D. Moore. Isometric immersions of space forms in space forms // Pacific J. Math. 1972. V. 40. P. $157-166$.

[118] J. D. Moore. Submanifolds of constant positive curvature. I // Duke Math. J. 1977. V. 44. № 2. P. 449-484.

[119] J. D. Moore. Isometric homotopy in codimension two // Trans. Amer. Math. Soc. 1985. V. 292. № 2. P. 653-663.

[120] J. D. Moore. On extendability of isometric immersions of spheres // Duke Math. J. 1996. V. 85. № 3. P. 685-699.

[121] H. Nakagawa, K. Ogiue. Complex space forms immersed in complex space forms // Trans. Amer. Math. Soc. 1976. V. 219. P. 289-297.

[122] J. Nash. $C^{1}$-isometric imbeddings // Ann. of Math. 1954. V. 3. P. 383-396. Рус. перев.: Дж. Нэш. $C^{1}$-изометрические вложения // Математика. 1957. Т. 1. № 2. С. 3-16.

[123] J. Nash. The imbedding problem for Riemannian manifolds // Ann. of Math. 1956. V. 63. № 1. Р. 20-63. Рус. перев.: Дж. Нэш. Проблема вложений для римановых многообразий // УМH. 1971. Т. 26. № 4. С. 173-216.

[124] Y. A. Nikolayevsky. A non-immersion theorem for a class of hyperbolic manifolds // Differential Geom. Appl. 1998. V. 9. № 3. P. 239-242.

[125] K. Nomizu. Isometric immersions of the hyperbolic plane into the hyperbolic space // Math. Ann. 1973. V. 205. P. 181-192.

[126] B. O’Neill. Umbilics of constant curvature immersions // Duke Math. J. 1965. V. 32. № 1. P. $149-159$

[127] T. Otsuki. On the existence of solutions of a system of quadratic equations and its geometrical application // Proc. Japan. Acad. 1953. V. 29. P. 99-100.

[128] F. J. Pedit. A non-immersion theorem for space forms // Comment. Math. Helv. 1988. V. 63. P. $672-674$.

[129] В.В.Пелипенко, Д. Д. Соколов. Внешне полные поверхности постоянной отрицательной кривизны в псевдоевклидовом пространстве $E_{2,1}^{3} / /$ УМН. 1984. Т. 39. № 4 . C. $175-176$.

[130] U. Pincall. Hopf tori in $S^{3} / /$ Invent. Math. 1985. V. 81. P. 379-386.

[131] А. В. Погорелов. Изгибание выпуклых поверхностей. М.: Наука, 1951.

[132] А. В. Погорелов. Поверхности ограниченной внешней кривизны. Харьков: Изд-во Харьков. ун-та, 1956.

[133] А. В. Погорелов. Внешняя геометрия вьпуклых поверхностей. М.: Наука, 1969. 
[134] Э. Г. Позняк. Изометрические погружения двумерных римановых метрик в евклидовы пространства // УМН. 1973. Т. 28. № 4. С. 47-76.

[135] Э. Г. Позняк. Изометрическое погружение в $E^{3}$ некоторых некомпактных областей плоскости Лобачевского // Матем. сб. 1977. Т. 102. № 1. С. 3-12.

[136] Э.Г. Позняк. Геометрическая интерпретация регулярных решений уравнения $z_{x y}=$ $\sin z / /$ Дифференц. уравнения. 1979. Т. 15. № 7. С. 1332-1336.

[137] Э. Г. Позняк, А. Г. Попов. Геометрия уравнения sin-Гордона // Итоги науки и техники. Проблемы геометрии. Т. 23. М.: ВИНИТИ, 1991. С. 99-130.

[138] Э. Г. Позняк, Д. Д. Соколов. Изометрические погружения римановых пространств в евклидовы пространства // Итоги науки и техники. Алгебра, геометрия, топология. Т. 15. М.: ВИНИТИ, 1977. С. 173-211.

[139] Э. Р. Розендорн. Реализация метрики $d s^{2}=d u^{2}+f^{2}(u) d v^{2}$ в пятимерном евклидовом пространстве // Докл. АН Арм. ССР. 1960. Т. 30. № 4. С. 197-199.

[140] Э. Р. Розендорн. К вопросу о погружении двумерных римановых метрик в четырехмерное евклидово пространство // Вестник МГУ. Сер. матем., мех. 1979. № 2. С. 47-50.

[141] Э. Р. Розендорн. Поверхности отрицательной кривизны // Итоги науки и техники. Совр. пробл. матем. Фундаментальные направления. Геометрия-3. Т. 48. М.: ВИНИТИ, 1985. С. $98-195$.

[142] И. Х. Сабитов. Регулярность выпуклых областей с регулярной в классах Гёльдера метрикой // Сиб. матем. журн. 1976. Т. 17. № 4. С. 907-915.

[143] И. Х. Сабитов. Изометрические погружения и вложения локально евклидовых метрик в $R^{3}$ // Сиб. матем. журн. 1985. Т. 26. №3. С. 156-167.

[144] И.Х. Сабитов. Изометрические погружения и вложения локально евклидовых метрик в $R^{2} / /$ Тр. семинара по векторному и тензорному анализу. МГУ. 1988. № 23. С. 147-156.

[145] И.Х. Сабитов. Об изометрических погружениях плоскости Лобачевского в $E^{4} / /$ Сиб. матем. журн. 1989. Т. 30. № 5. С. 179-186.

[146] И.Х.Сабитов. Изометрические погружения и вложения локально евклидовых метрик в $R^{2}$ // Изв. РАН. Сер. матем. 1999. Т. 63. №6. С. 147-167.

[147] И.Х. Сабитов, С. З. Шефель. О связях между порядками гладкости поверхности и ее метрики // Сиб. матем. журн. 1976. Т. 17. № 4. С. 916-925.

[148] R. Sasaki. Solitons equations and pseudospherical surfaces // Nuclear Phys. 1979. V. 154. P. 343-357.

[149] Г. С. Шефель. Геометрические группы преобразований евклидова пространства // Докл. АН СССР. 1984. Т. 277. № 4. С. 803-806.

[150] Г. С. Шефель. Группы преобразований евклидова пространства // Сиб. матем. журн. 1985. T. 26. № 3. C. 197-215.

[151] Г. С. Шефель. О двух классах $k$-мерных поверхностей в $n$-мерном пространстве // Сиб. матем. журн. 1969. Т. 10. № 2. С. 459-466.

[152] С. З. Шефрель. Вполне регулярные изометрические погружения в евклидово пространство // Сиб. матем. журн. 1970. Т. 11. № 2. С. 442-460.

[153] С. З. Шефель. $C^{1}$-гладкие изометричные погружения // Сиб. матем. журн. 1974. Т. 15. №6. C. $1372-1393$.

[154] С. З. Шефель. $C^{1}$-гладкие поверхности ограниченной внешней положительной кривизны // Сиб. матем. журн. 1975. Т. 16. № 5. С. 1122-1123.

[155] С.3. Шефель. Геометрические свойства погруженных многообразий // Сиб. матем. журн. 1985. Т. 26. № 1. C. 170-188.

[156] F. Shur. Über die Deformation der Räume constanter Riemannschen Krümunges Masses // Math. Ann. 1886. V. 27. P. 163-176.

[157] Д. Д. Соколов. О строении предельного конуса выпуклой поверхности в псевдоевклидовом пространстве // УМН. 1975. Т. 30. №1. С. 261-262.

[158] Д. Д. Соколов. Поверхности в псевдоевклидовом пространстве // Итоги науки и техники. Проблемы геометрии. Т. 11. М.: ВИНИТИ, 1980. С. 177-201. 
[159] M. Spivak. A Comprehensive Introduction to Differential Geometry. V. 4. Boston: Publish or Perish, 1975.

[160] M. Spivak. Some left over problems from classical differential geometry // Proc. Sympos. Pure Math. 1975. V. 27. P. 245-252.

[161] K. Tenenblat, C.-L. Terng. Bäcklund's theorem for $n$-dimensional submanifolds of $R^{2 n-1} / /$ Ann. of Math. (2). 1980. V. 111. P. 477-490.

[162] C.-L. Terng. A higher dimension generalization of the Sine-Gordon equation and its soliton theory // Ann. of Math. (2). 1980. V. 111. № 3. P. 491-510.

[163] C.-L. Terng. Soliton equations and differential geometry // J. Differential Geom. 1997. V. 45. P. $405-407$.

[164] C.-L. Terng, K. Uhlenbeck. Geometry of solitons // Notices Amer. Math. Soc. 2000. №1. P. $17-25$.

[165] C. Tompkins. A flat Klein bottle isometrically embedded in Euclidean 4-space // Bull. Amer. Math. Soc. 1941. V. 47. № 6. P. 508.

[166] G. Toth. Eigenmaps and the space of minimal immersions between spheres // Indiana Univ. Math. J. 1997. V. 46. №2. P. 637-658.

[167] G. Toth. New construction for spherical minimal immersions // Geom. Dedicata. 1997. V. 67. P. $187-196$.

[168] G. Toth. Infinitesimal rotations of isometric minimal immersions between spheres // Amer. J. Math. 2000. V. 122. P. 117-152.

[169] V. Ushakov. The explicit general solution of trivial Monge-Ampere equation // Comment. Math. Helv. 2000. V. 75. № 1. P. 125-133.

[170] Ю. А. Волков, С. М. Владимирова. Изометрические погружения евклидовой плоскости в пространство Лобачевского // Матем. заметки. 1971. Т. 10. № 3. С. 327-332.

[171] Л. И. Воробьева. Невозможность $C^{2}$-изометрического погружения в $E^{3}$ полуплоскости Лобачевского // Вестник МГУ. Сер. матем., мех. 1976. № 5. С. 42-46.

[172] J. L. Weiner. Flat tori in $S^{3}$ and their Gauss maps // Proc. London Math. Soc. 1991. V. 62. № 1. P. 54-76.

[173] J. L. Weiner. Rigidity of Clifford tori // Geometry and Topology of Submanifolds (Leuven, Brussels, 1994). Singapore: World Scientific, 1995. P. 274-277.

[174] L. Whitt. Isometric homotopy and codimension-two isometric immersions of the $n$-sphere into Euclidean space // J. Differential Geom. 1979. V. 14. № 2. P. 295-302.

[175] J. A. Wolf. Homogeneous manifolds of constant curvature // Comment. Math. Helv. 1961. V. 36. № 2. P. 112-148.

[176] F. Xavier. A non-immersion theorem for hyperbolic manifolds // Comment. Math. Helv. 1985. № 60. P. 280-283.

[177] A. Zeghib. Feuilletages géodésiques appliqués // Math. Ann. 1994. V. 298. P. 729-759.

[178] A. Zeghib. Feuilletages géodésiques des variétés localement symétriques // Topology. 1997. V. 36. № 4. P. 805-828. 